\title{
Determination of Peptide Backbone Torsion Angles using Double-Quantum Dipolar Recoupling Solid-State NMR Spectroscopy
}

\author{
Manish A. Mehta, Matthew T. Eddy, Seth A. McNeill, Frank D. Mills, Joanna R. Long*
}

Department of Chemistry and Biochemistry, 119 Woodland Street, Oberlin College, Oberlin, Ohio 44074

Department of Biochemistry and Molecular Biology and McKnight Brain Institute, University of Florida, Gainesville, Florida 32611

AUTHOR EMAIL ADDRESSES: manish.mehta@oberlin.edu, jrlong@mbi.ufl.edu

\section{Supplementary data}

Included in this file are:

1) The complete pulse sequence and phase cycle used in the DQ buildup and 2D DQ DRAWS experiments.

2) Experimental and theoretical powder diffraction spectra for the tripeptides.

3) The 2D DQ DRAWS spectra for the tripeptides with projections of the $f_{2}$ (CPMAS) and $f_{1}$ (DQDRAWS) dimensions and expansions of the isotropic peaks.

4) Fits of the $2 D$ DQ DRAWS data, including

a. A comparison of data ( $\mathrm{T}_{1}$ and fourier series) to simulations with a 10x10 degree grid of the full $\phi, \psi$ space using CSA parameters determined for each compound.

b. A comparison of data ( $\mathrm{T}_{1}$ and fourier series) to simulations with a $2 \times 5$ degree grid of $\phi, \psi$ space with $\phi$ within $\pm 10^{\circ}$ of the DQbuildup determined value using CSA parameters determined for each compound.

c. A comparison of data ( $\mathrm{T}_{1}$ and fourier series) to simulations with a $2 \mathrm{x} 5$ degree grid of $\phi, \psi$ space with $\phi$ within $\pm 10^{\circ}$ of the DQbuildup determined value using CSA parameters determined for each compound.

d. A comparison of data ( $\mathrm{T}_{1}$ and fourier series) to simulations with a $2 \mathrm{x} 5$ degree grid of $\phi, \psi$ space with $\phi$ varying over a slightly larger range and using generalized CSA parameters for peptidyl carbonyl carbons.

e. Example best fits using $\mathrm{T}_{1}$ and fourier series approaches. 
2D Double-Quantum DRAWS Experiments: The pulse diagram of the two-dimensional double-quantum DRAWS experiment is given by:
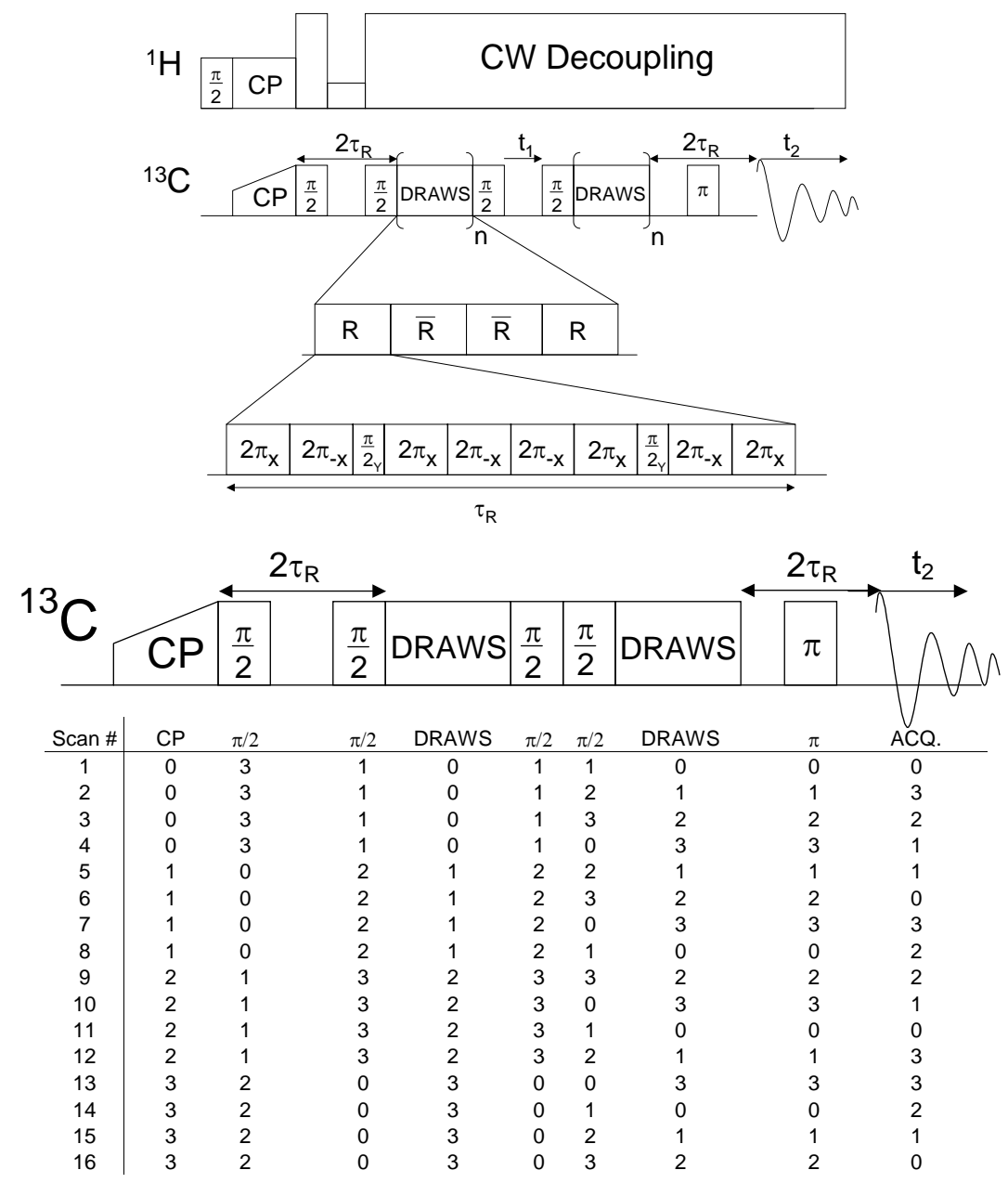

A 16-step phase cycle was used to select the double-quantum signal in both the buildup and the $2 \mathrm{D}$ experiments. The phases are given in increments of 90 degrees $\left(0=0^{\circ}, 1=90^{\circ}, 2=180^{\circ}\right.$, $3=270^{\circ}$ ). For the 2D-DQDR experiment, quadrature detection was achieved in the $t_{1}$ period, using the States-Haberkorn-Ruben method, by adding 45 degrees to each event before $t_{1}$ in the 16-step phase cycle to generate the imaginary portion of the signal. 


\section{GAF}
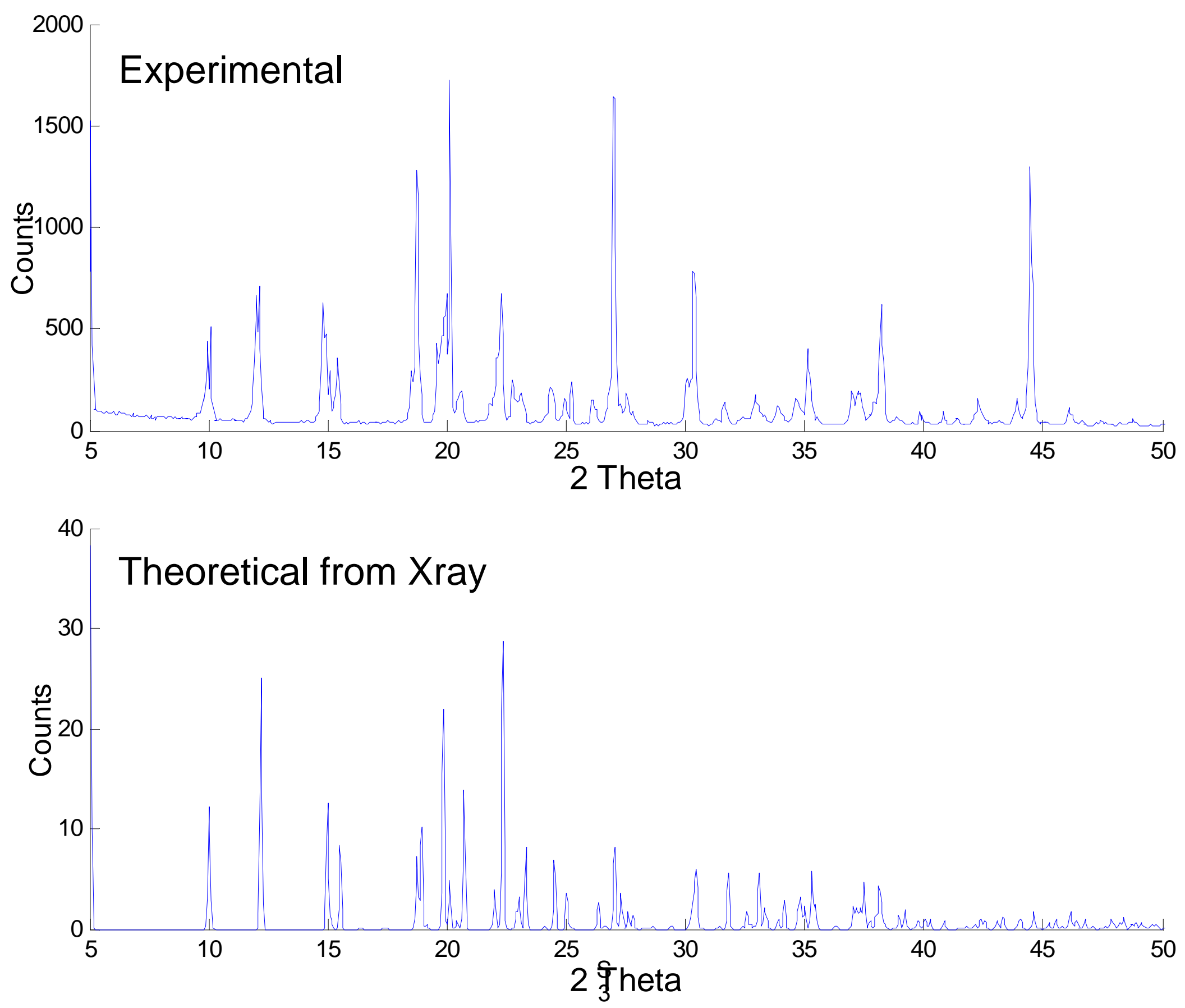


\section{GGV}
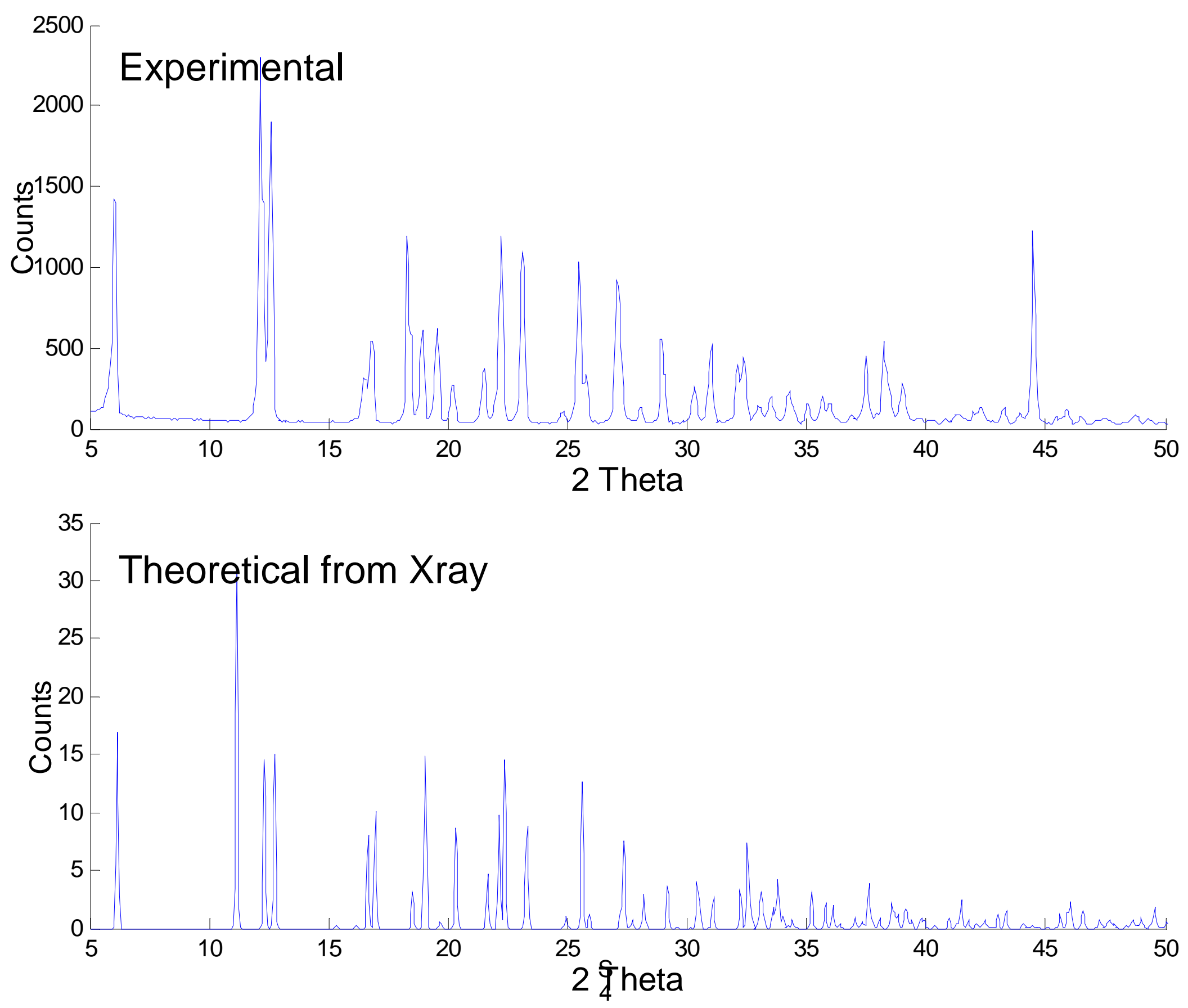


\section{AGG}
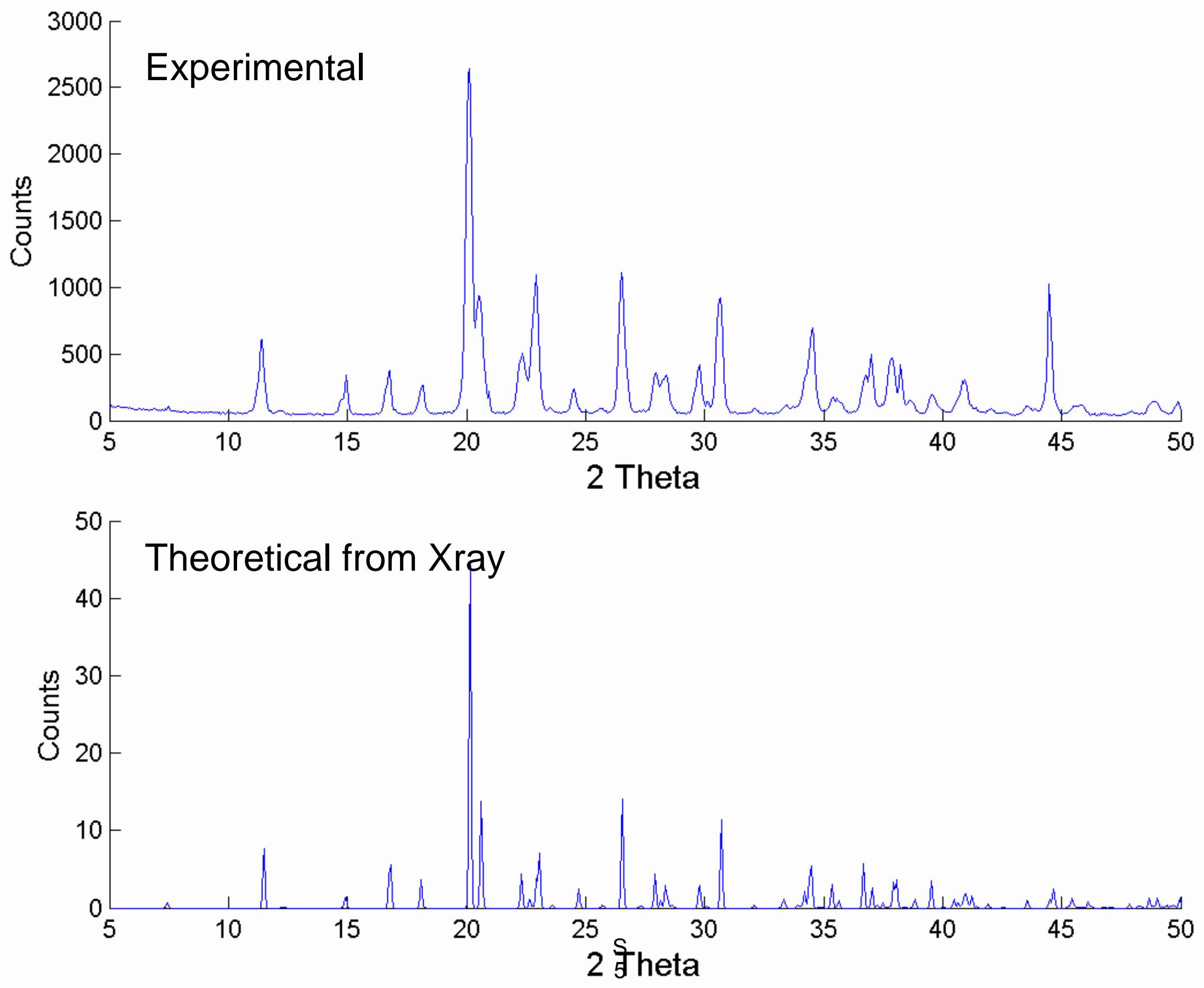


\section{FAG}
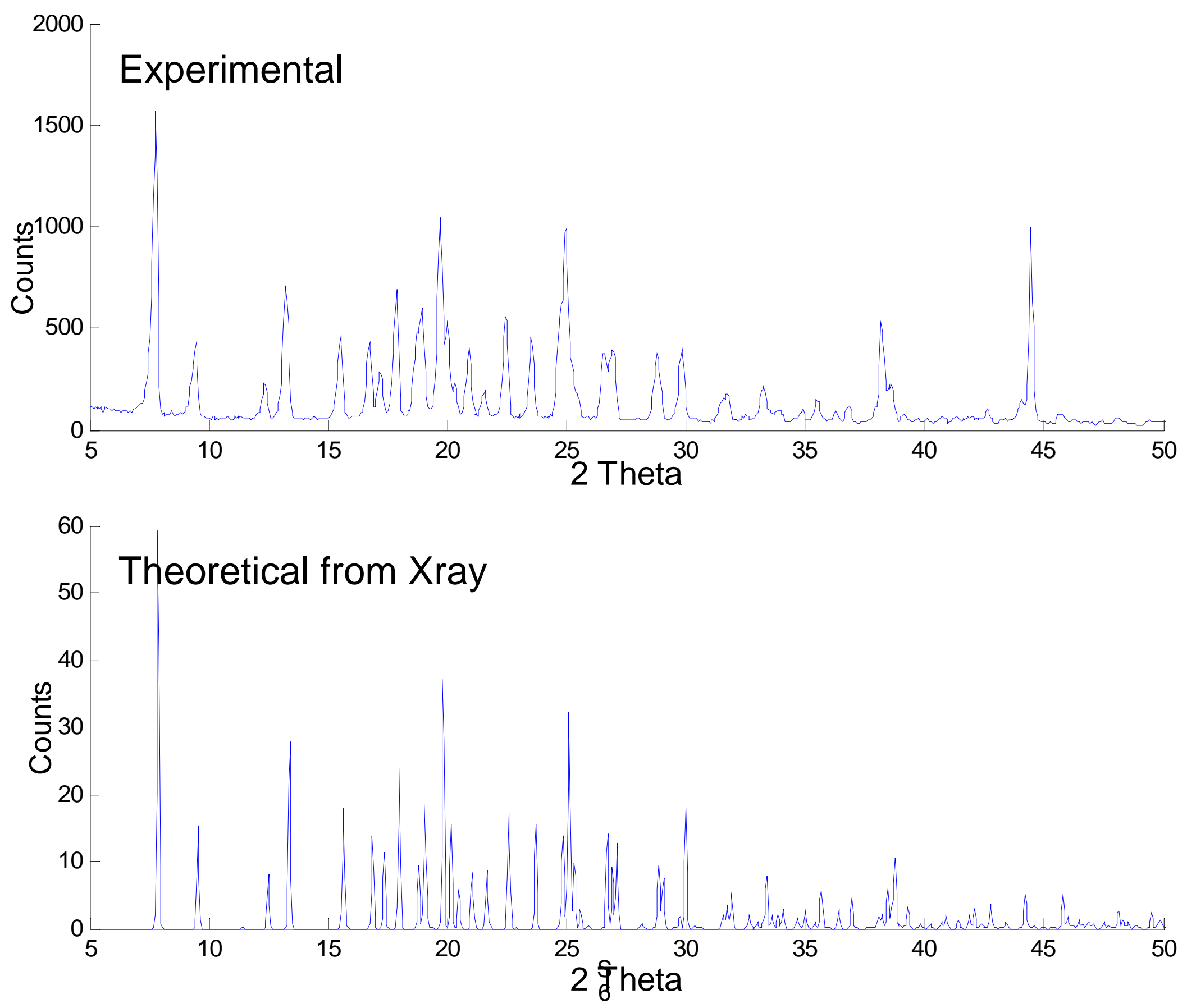

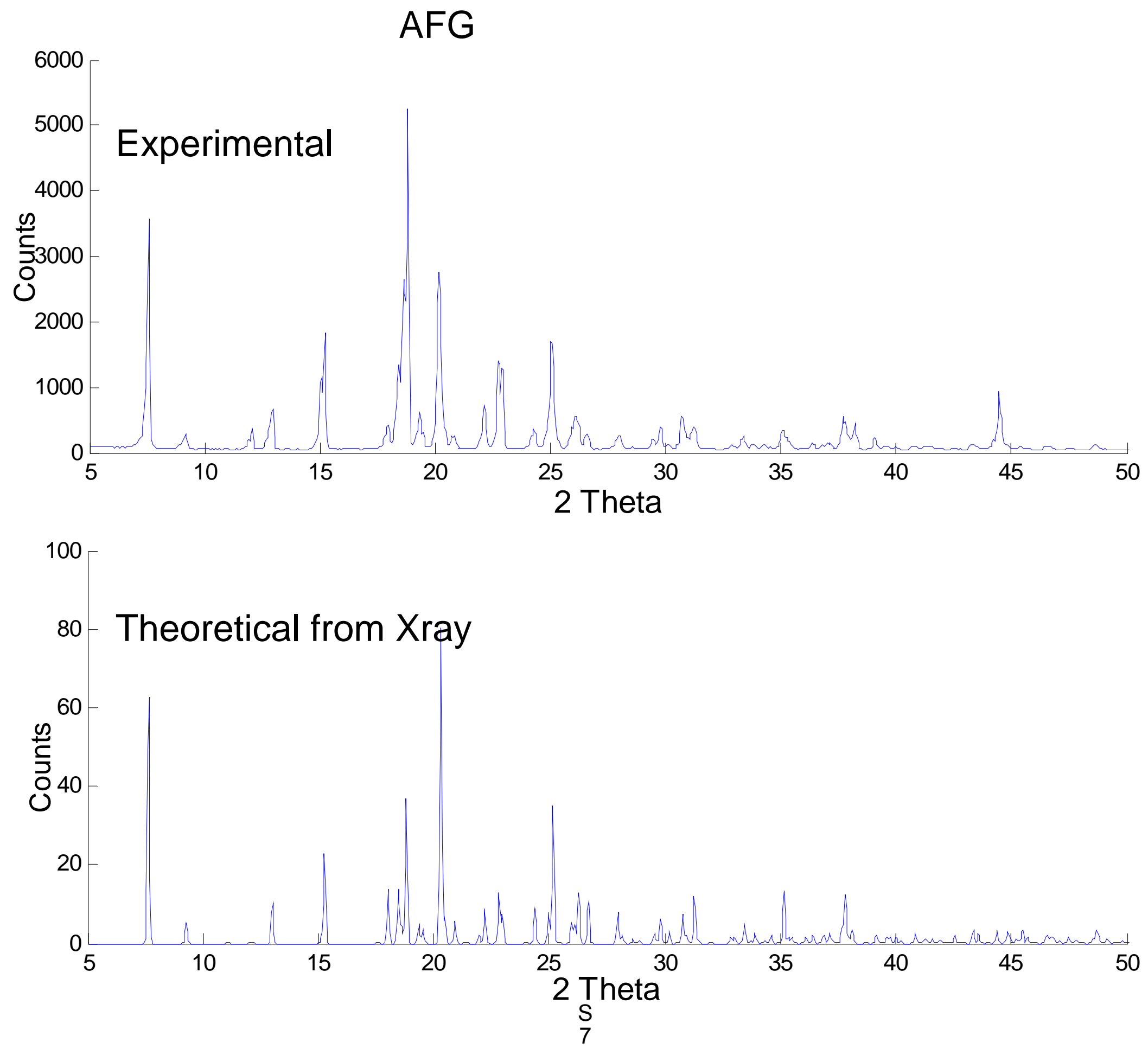


\section{GFF}
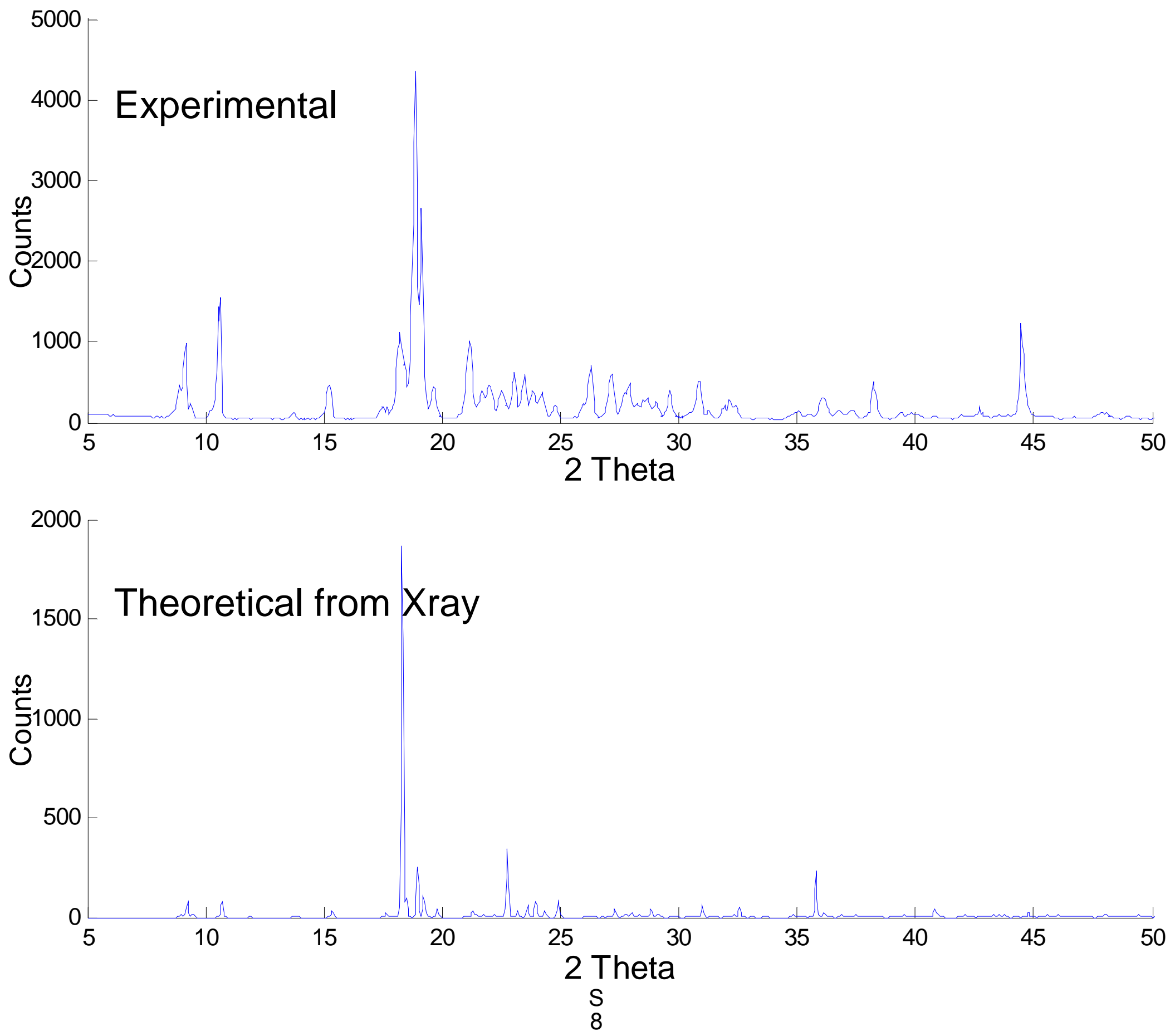
GFG
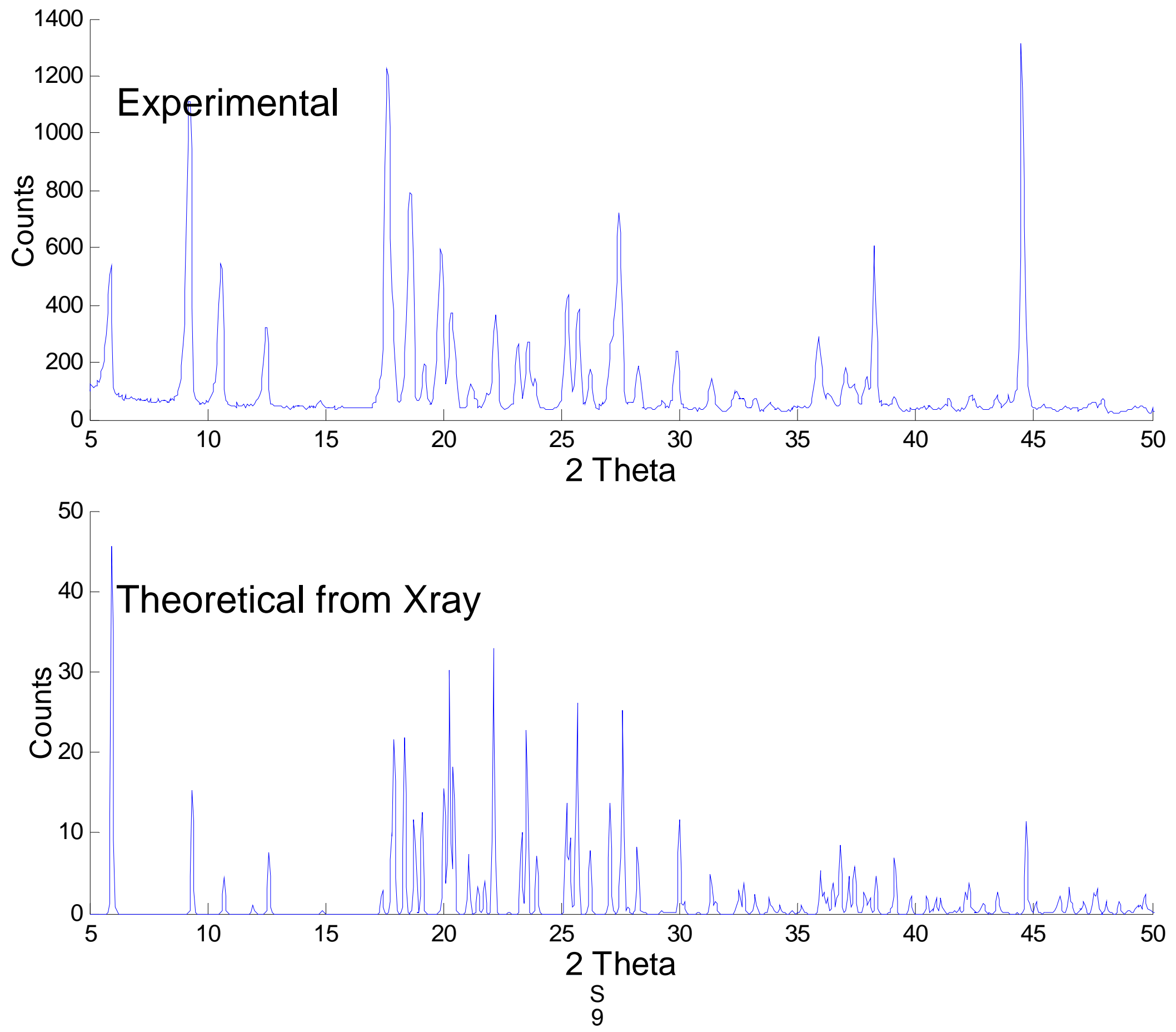


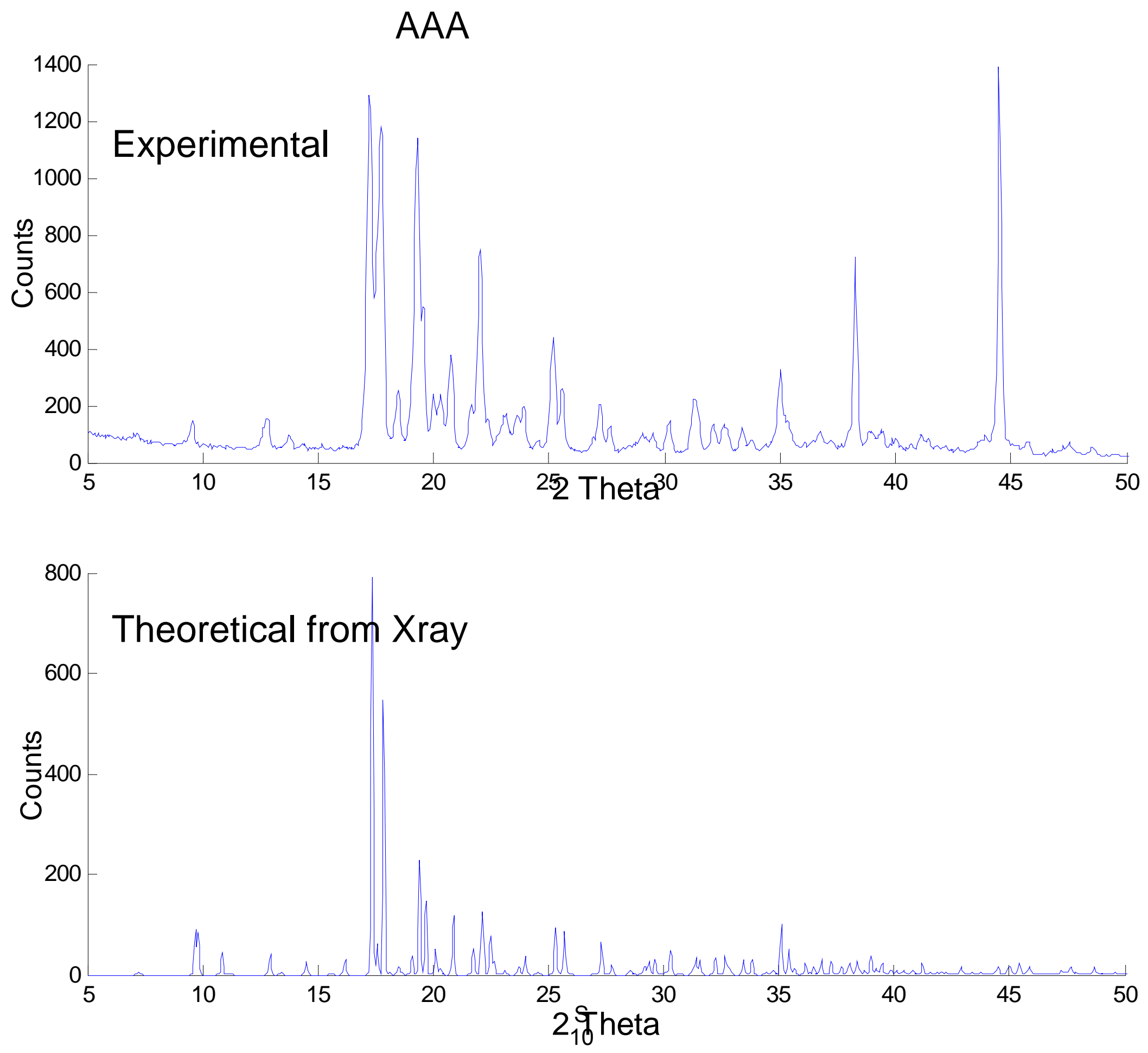


VGG

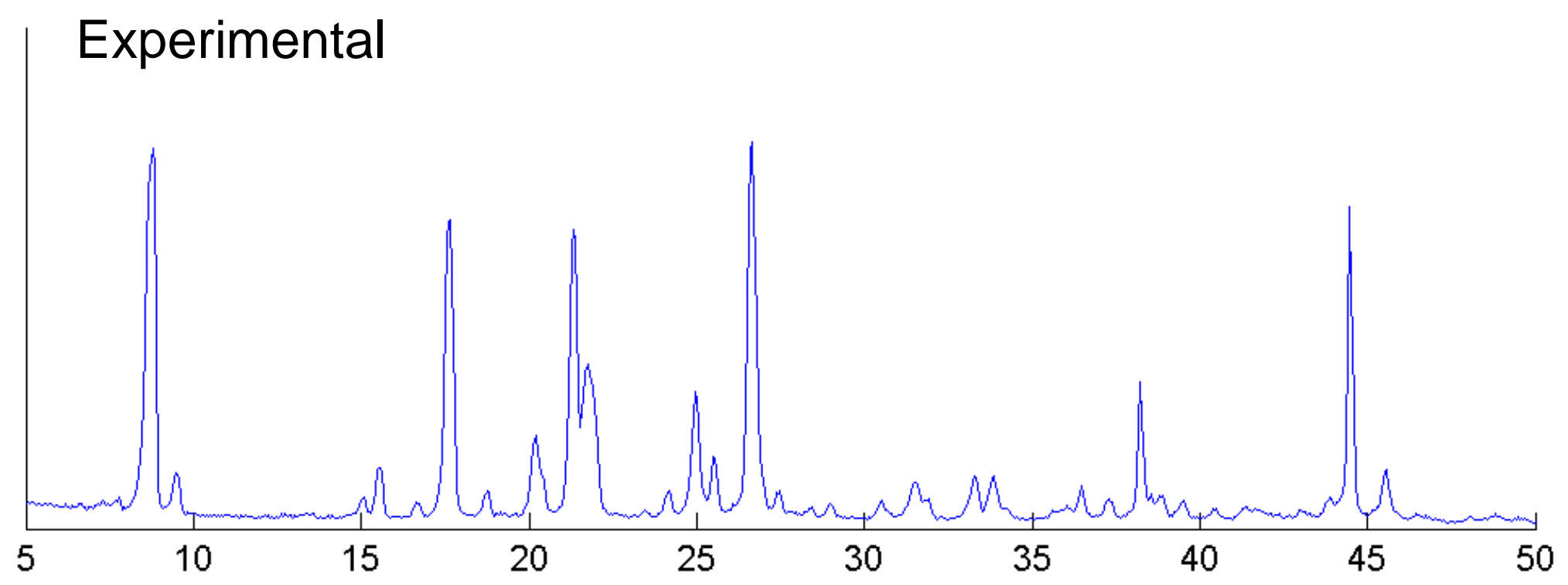

Theoretical from Xray

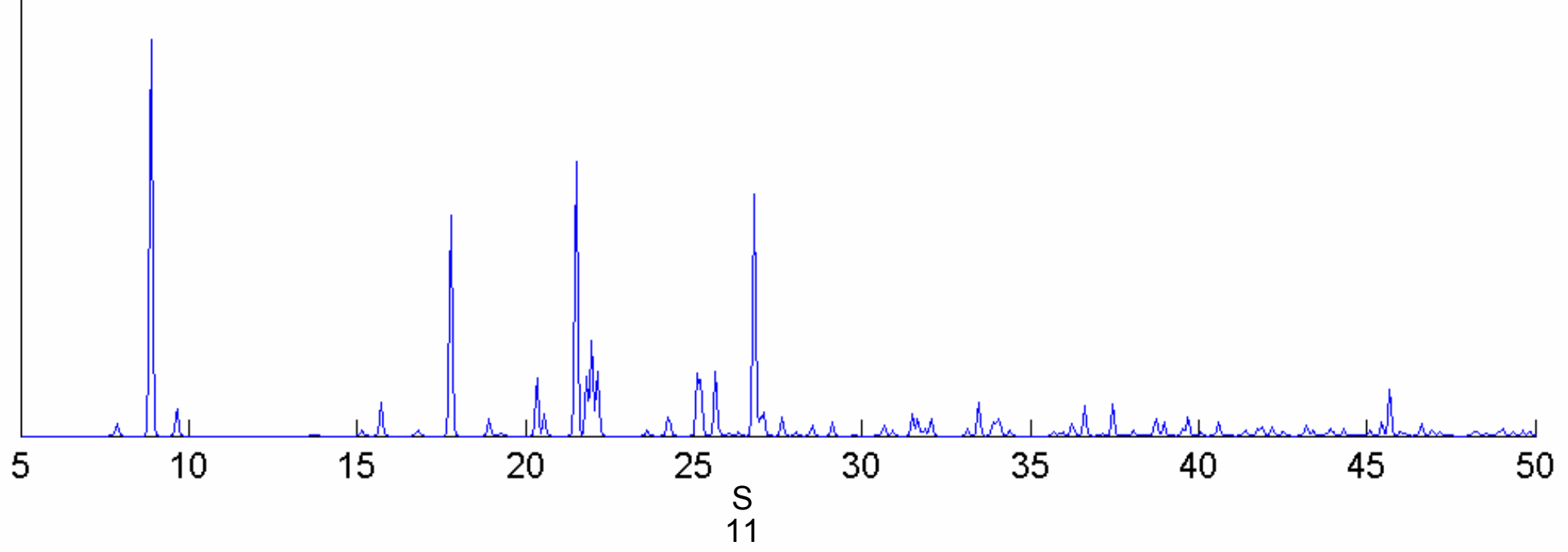




\section{GAV}

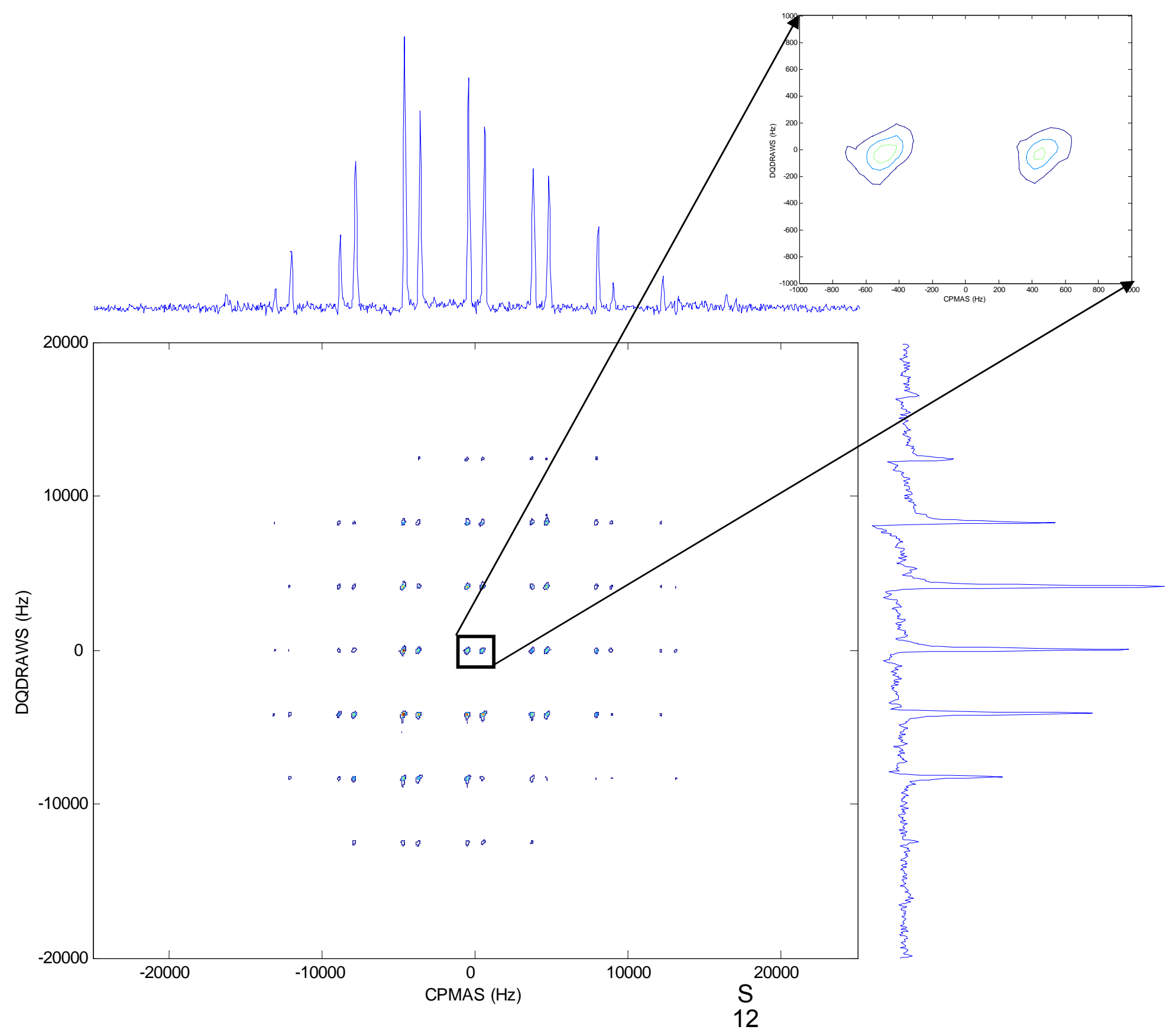




\section{GAF}

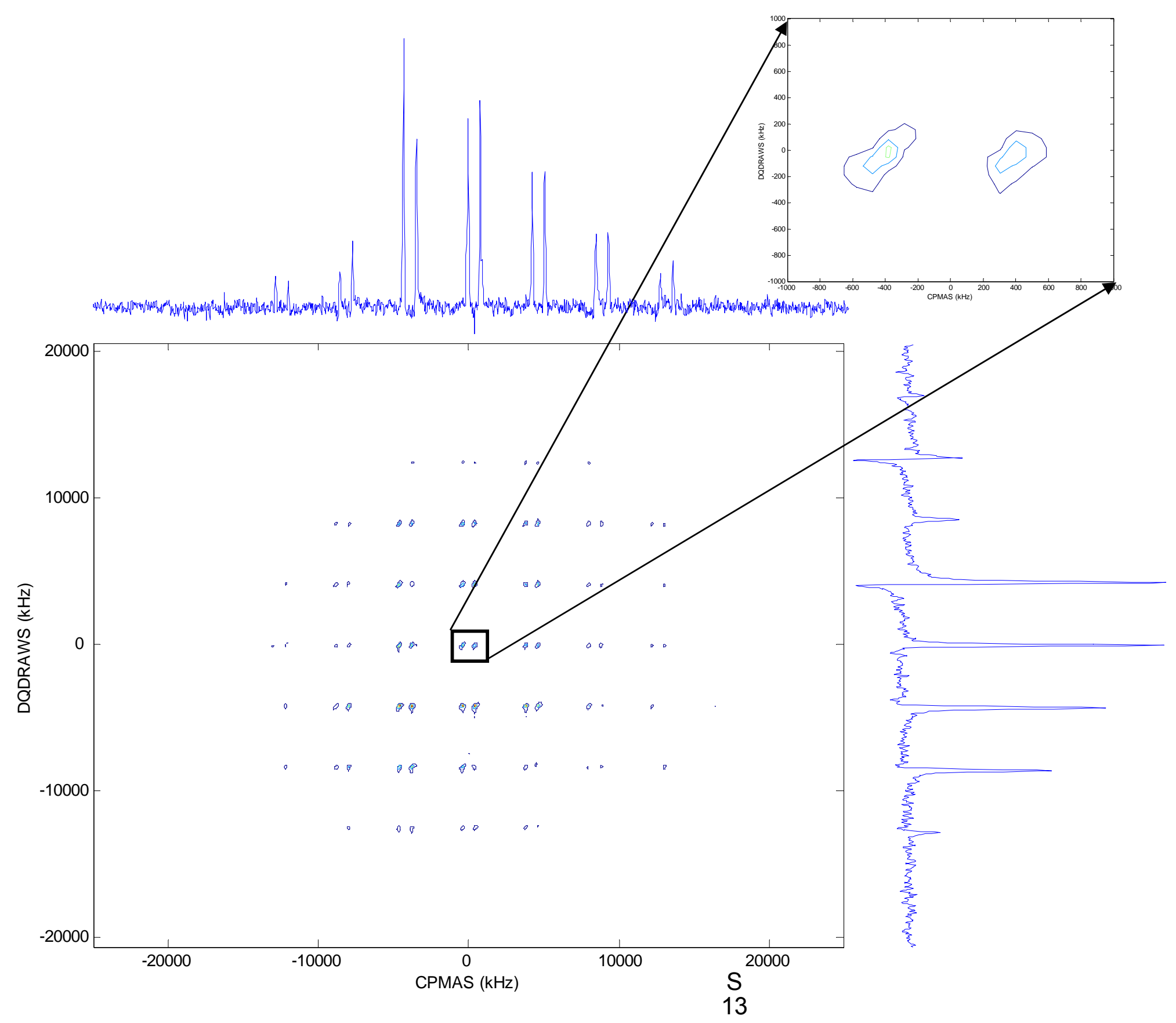




\section{GGV}

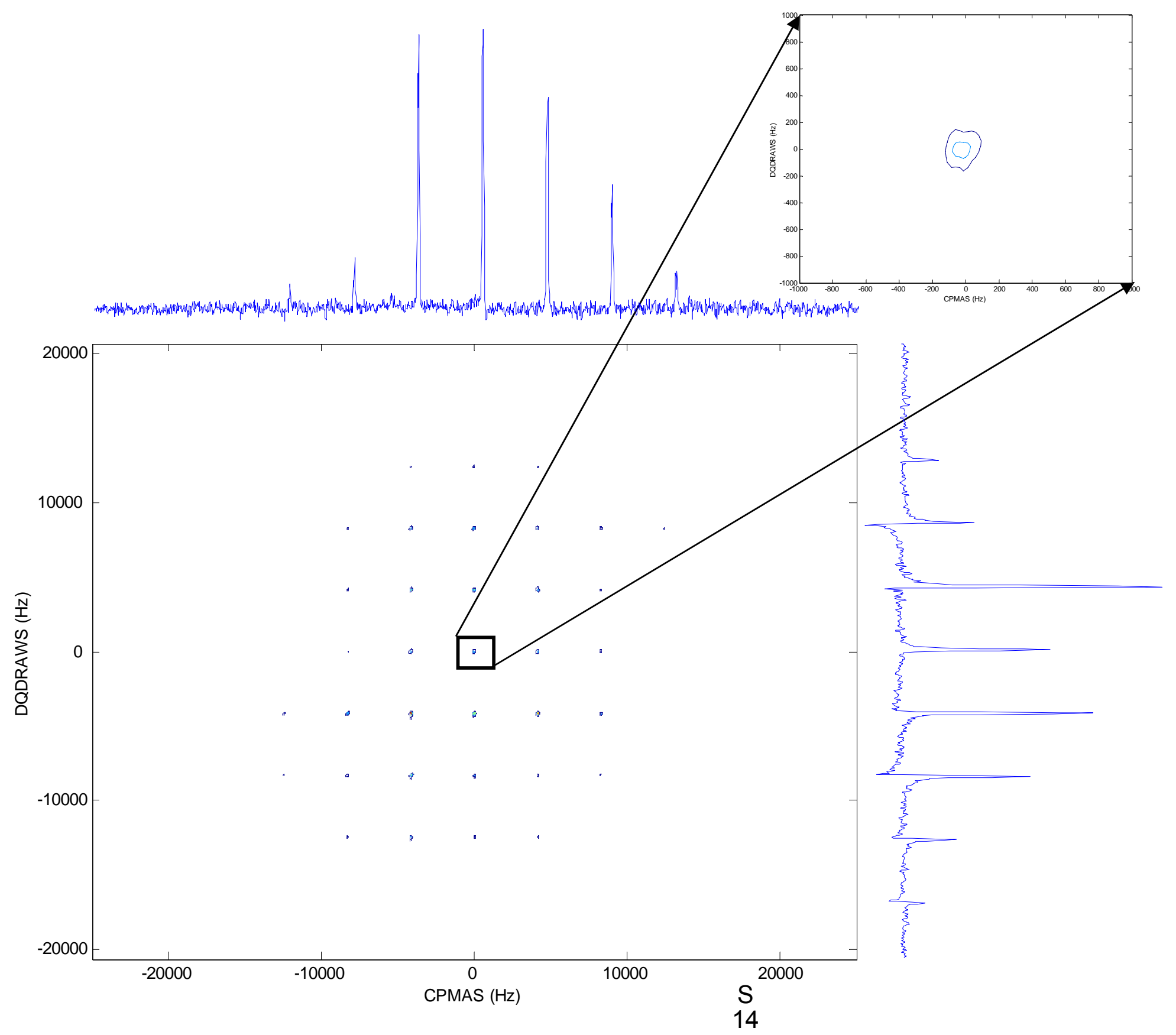




\section{AGG}

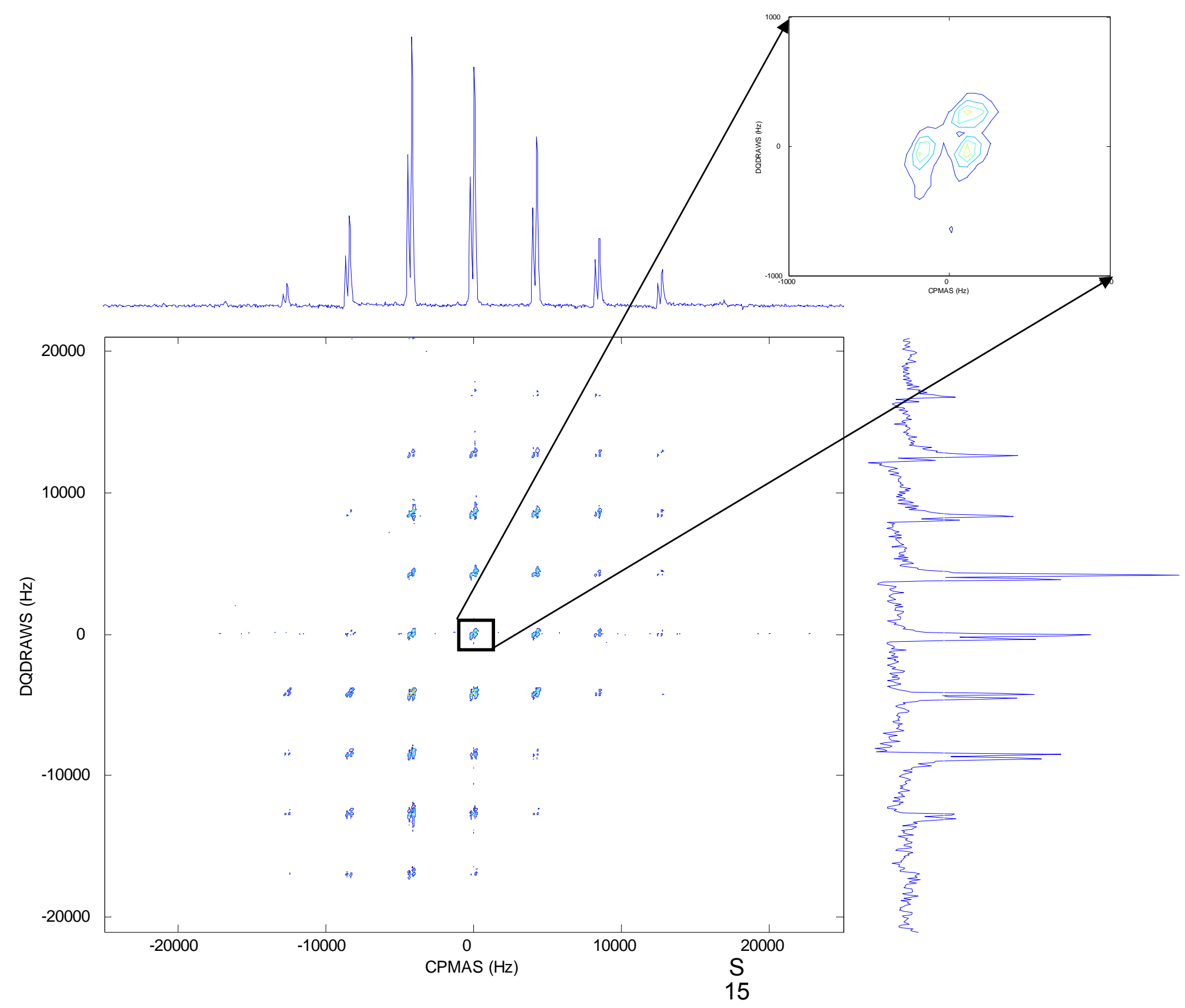




\section{FAG}

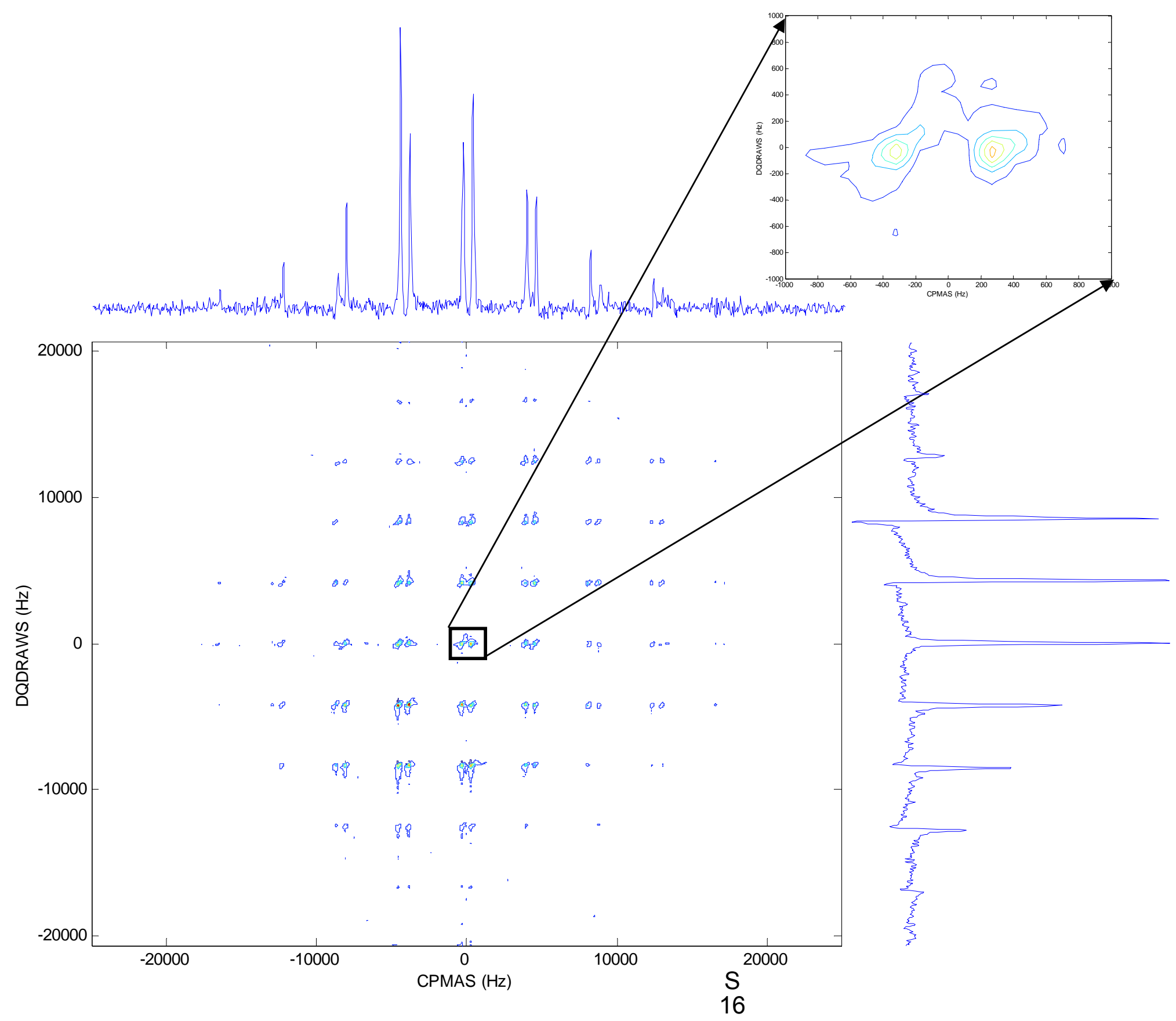




\section{AFG}

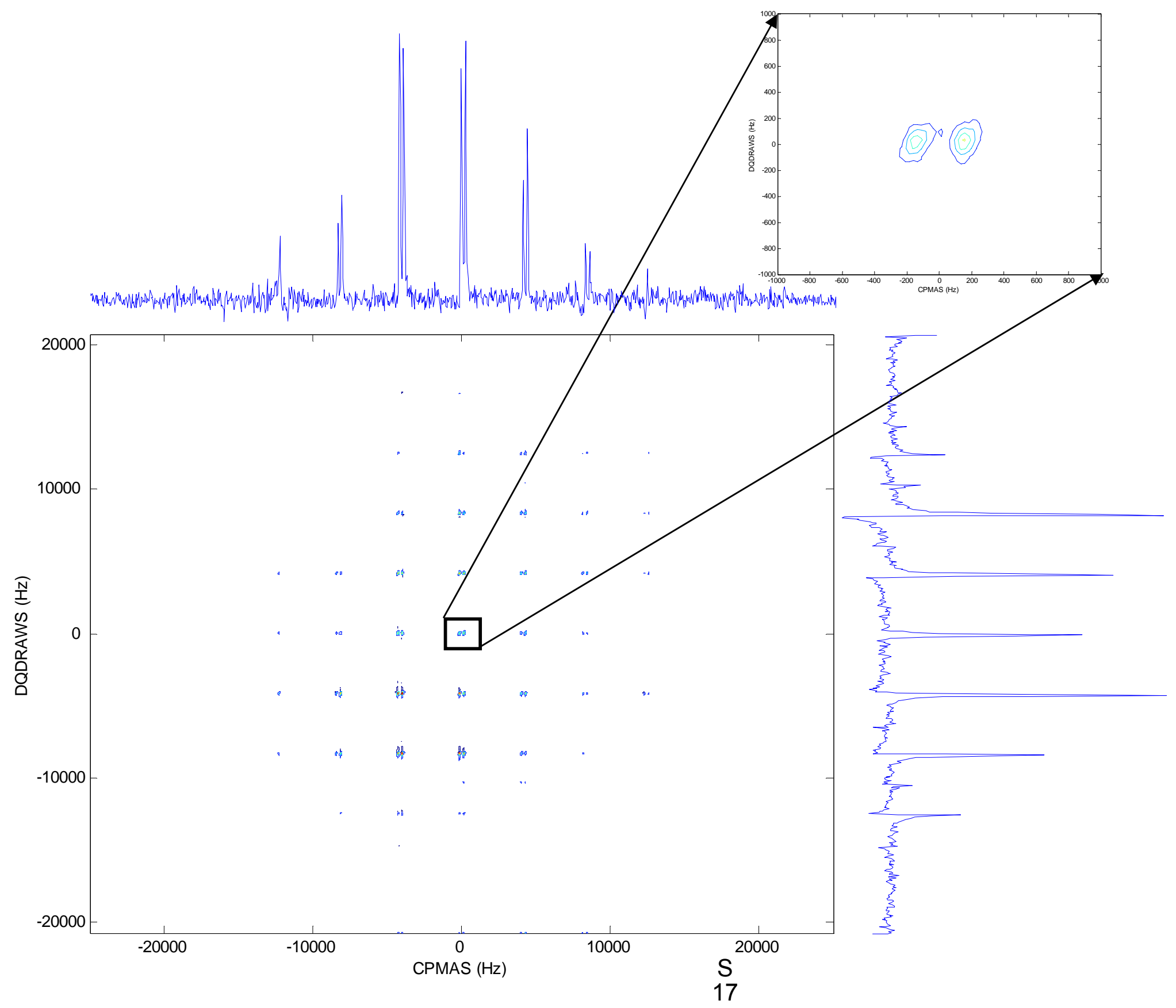




\section{GFF}

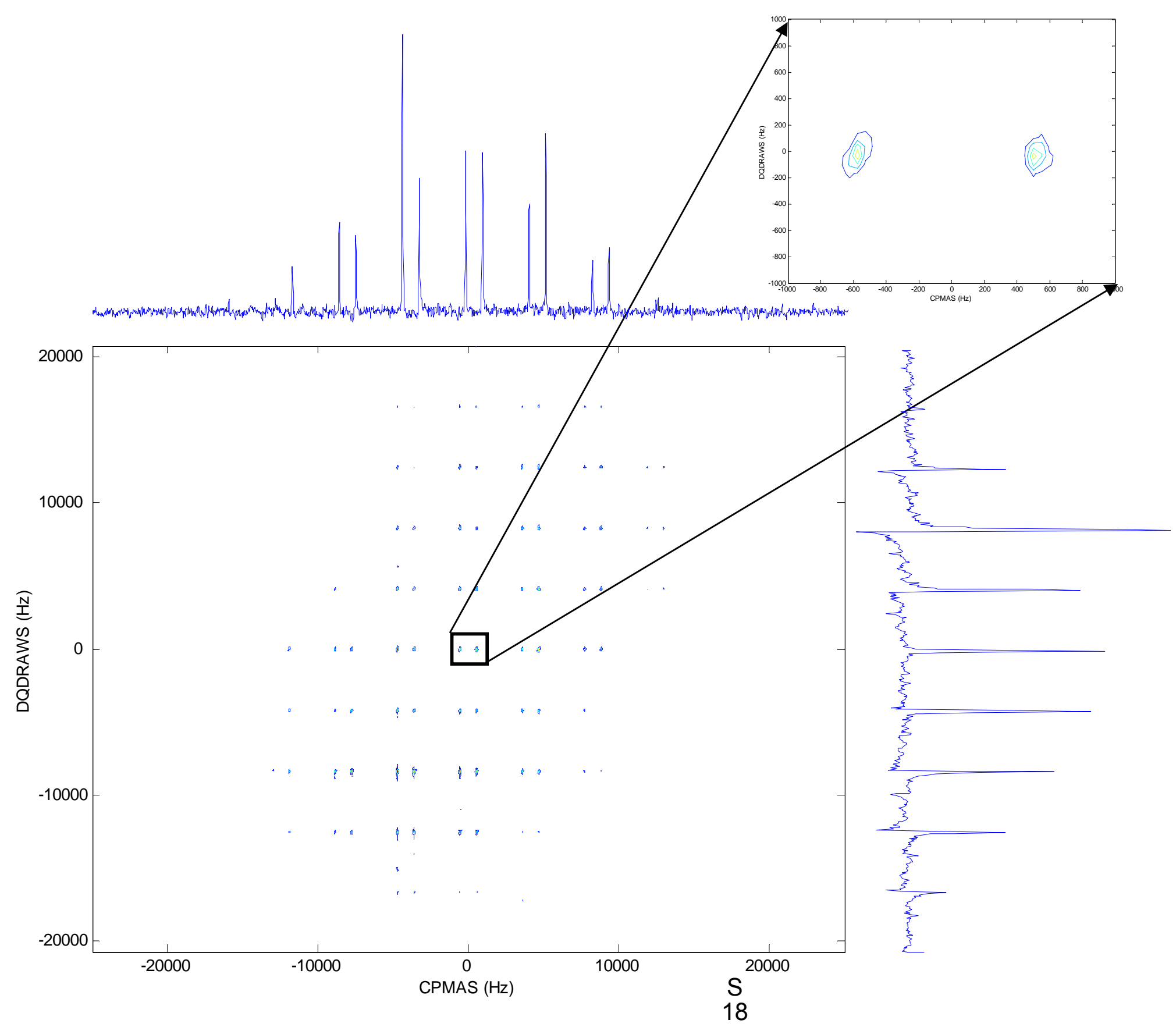




\section{GFG}

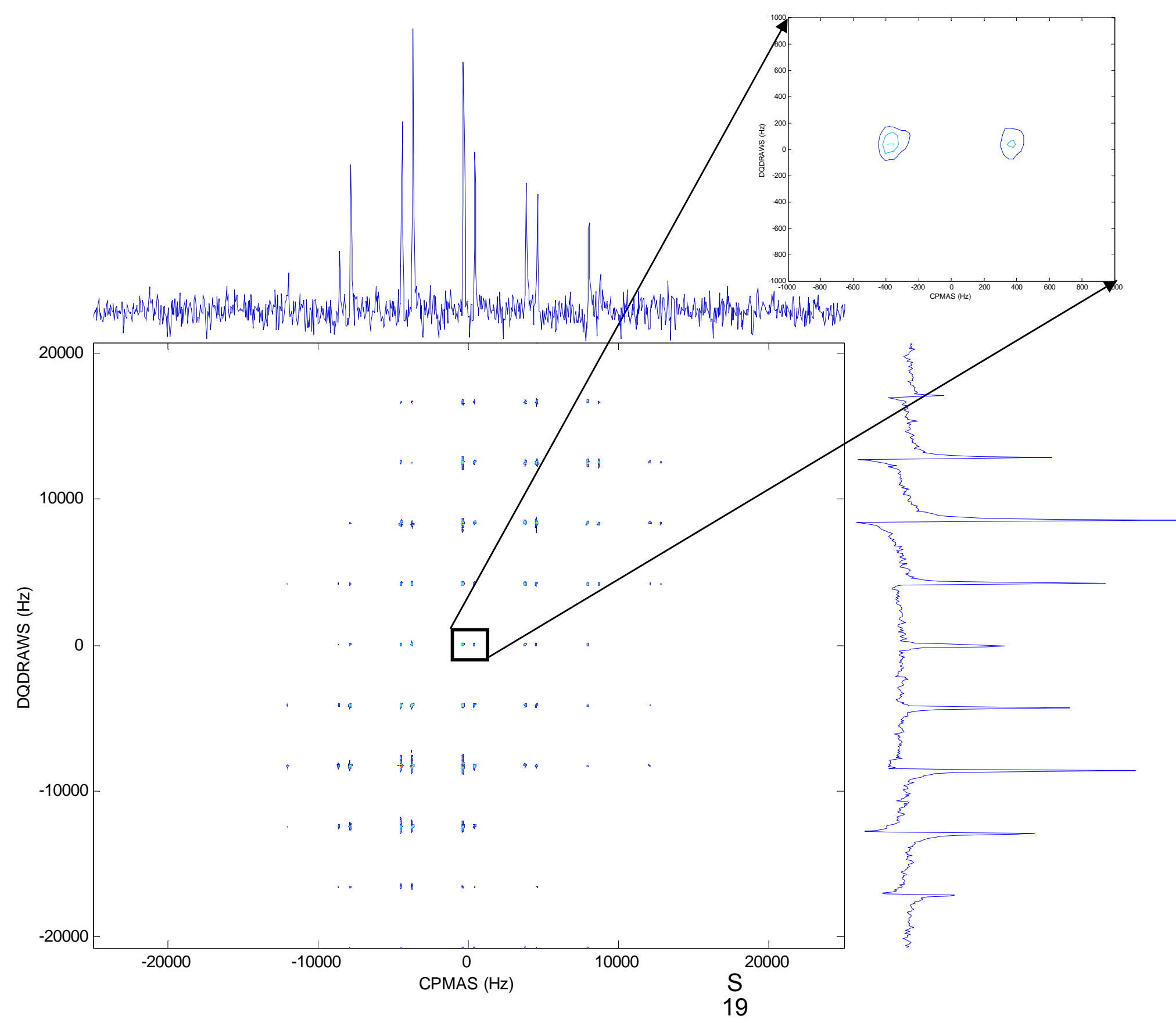




\section{VGG}

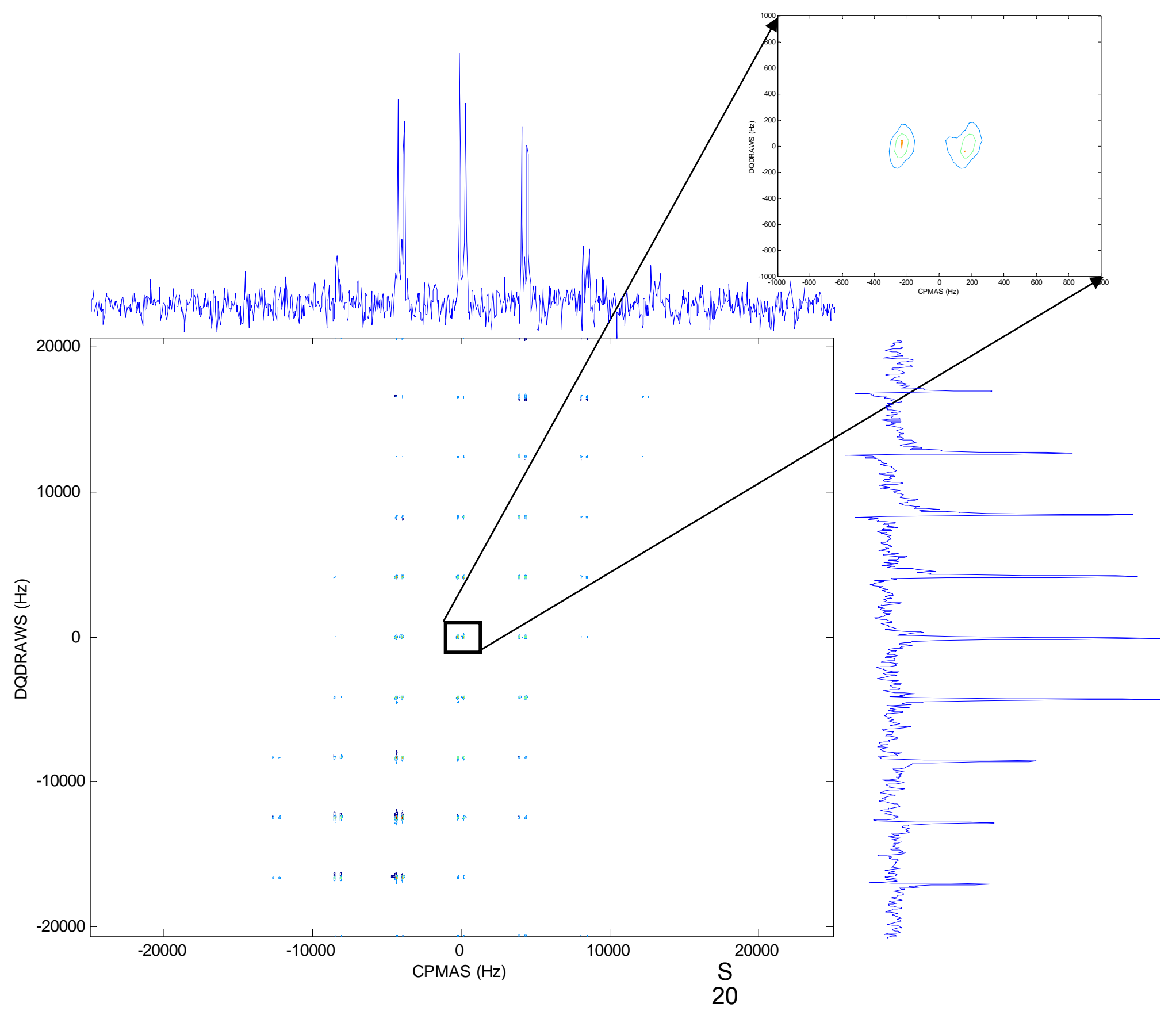


AAA

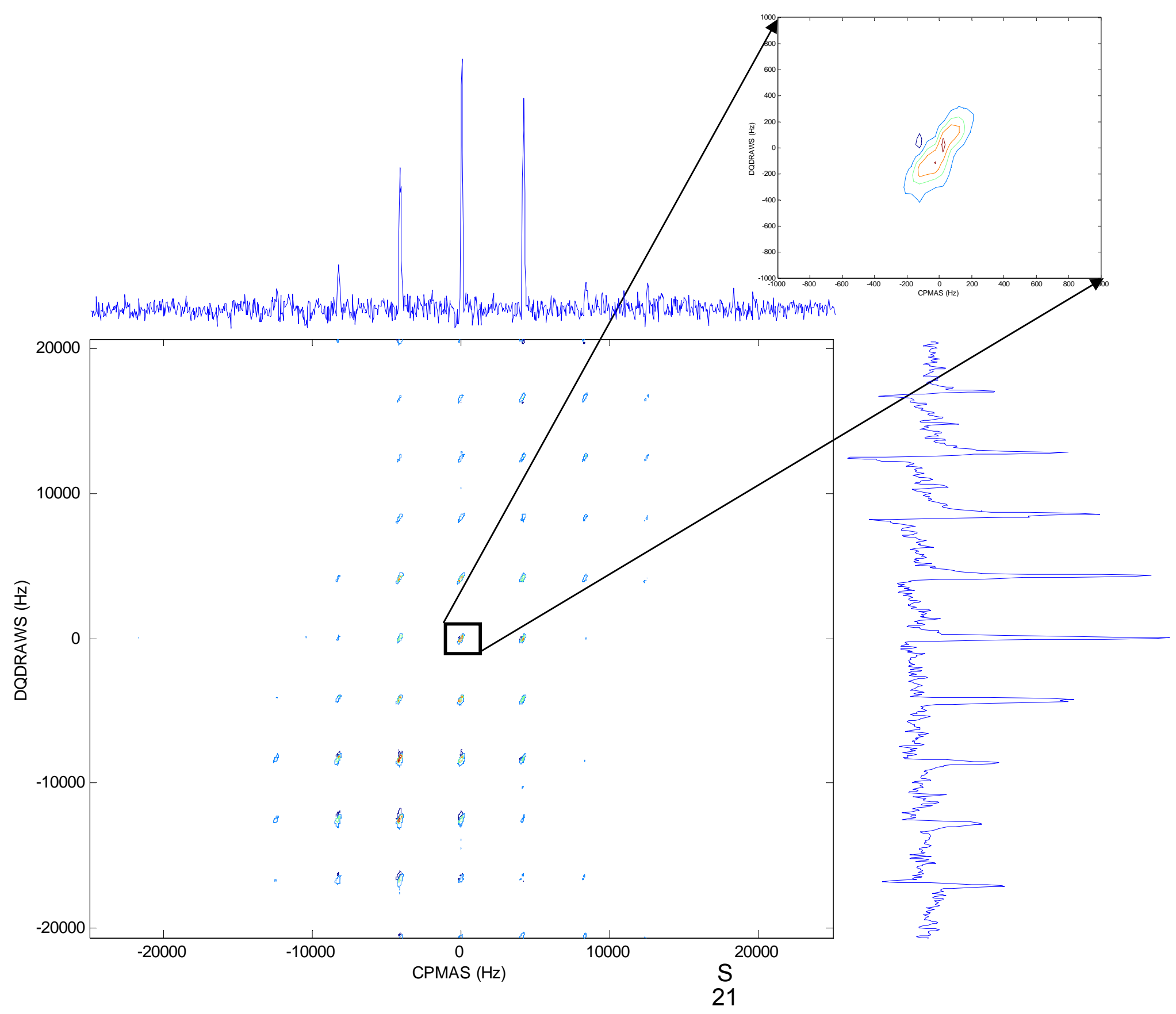




\section{0 degree GAV grid}

\section{Fourier Series}

Ramachandran $\chi^{2}$ map for GAV with step of 10 degrees Minimum at: $\phi=-70, \psi=-30$

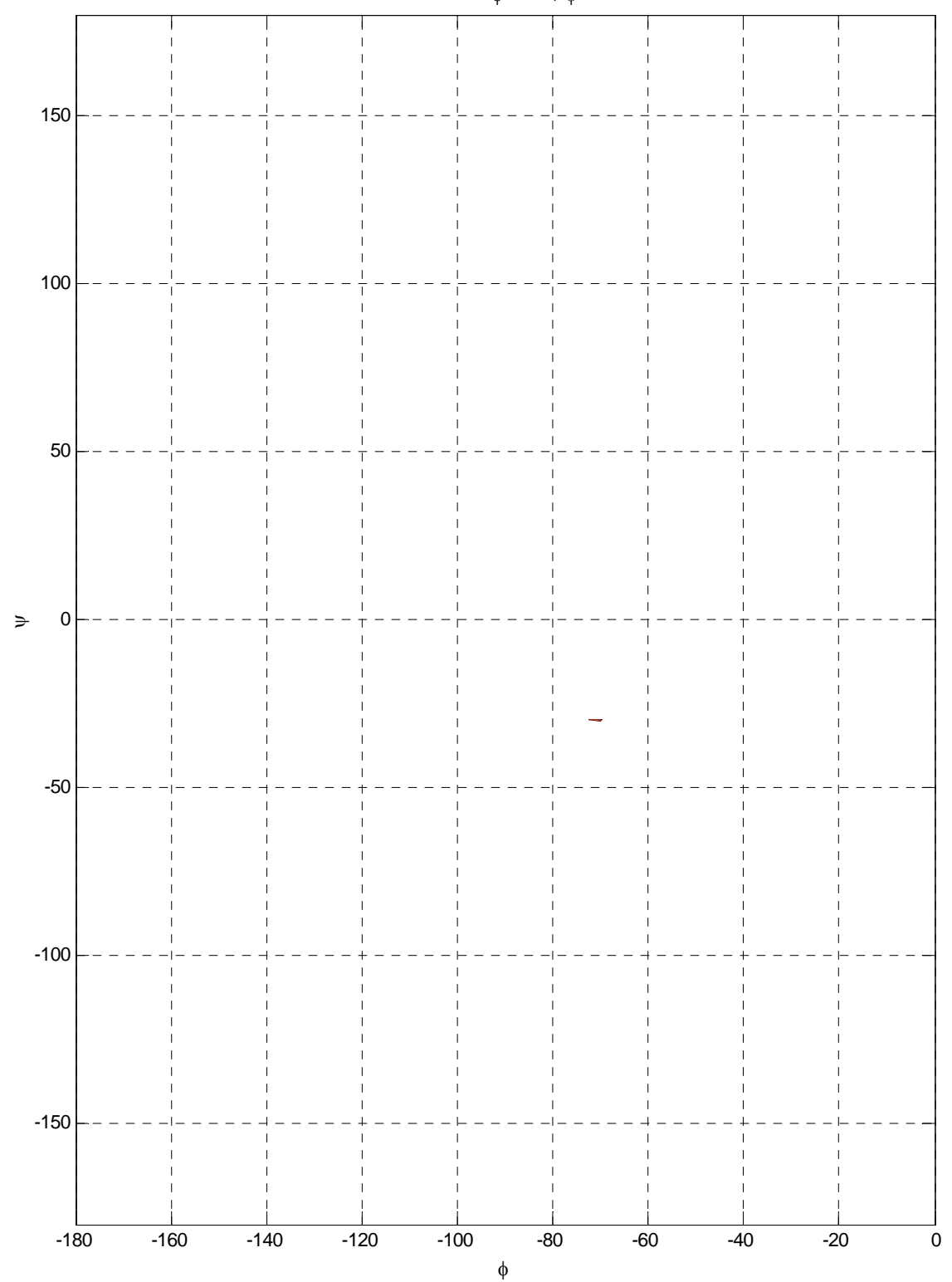

$t_{1}$ fit

Ramachandran $\chi^{2}$ map for GAV with step of 10 degrees Minimum at: $\phi=-70, \psi=-30$

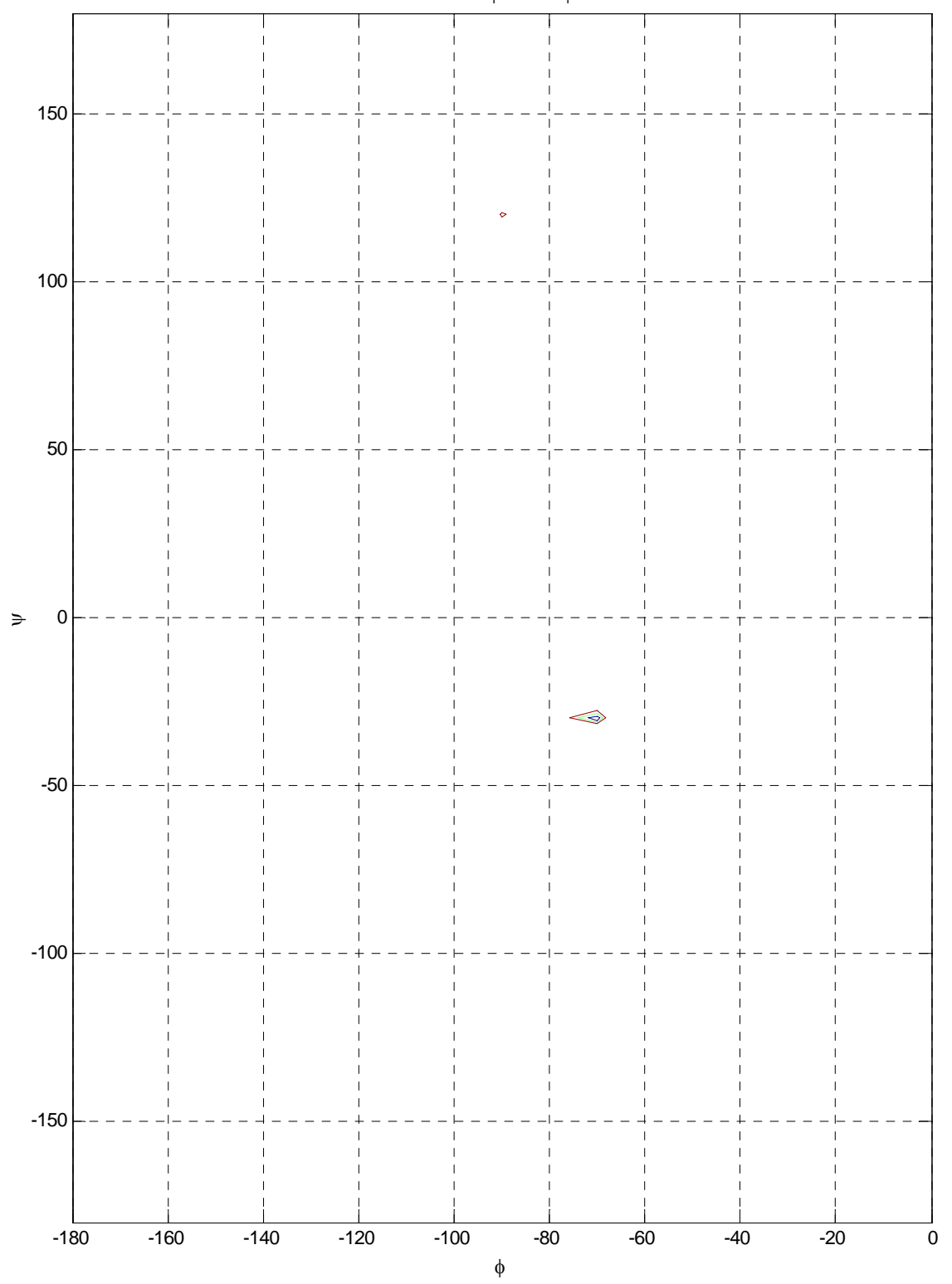




\section{5×2 degree GAV grid}

\section{Fourier Series}

Ramachandran $\chi^{2}$ map for GAV with step of 5 degrees Minimum at: $\phi=-69, \psi=-32$

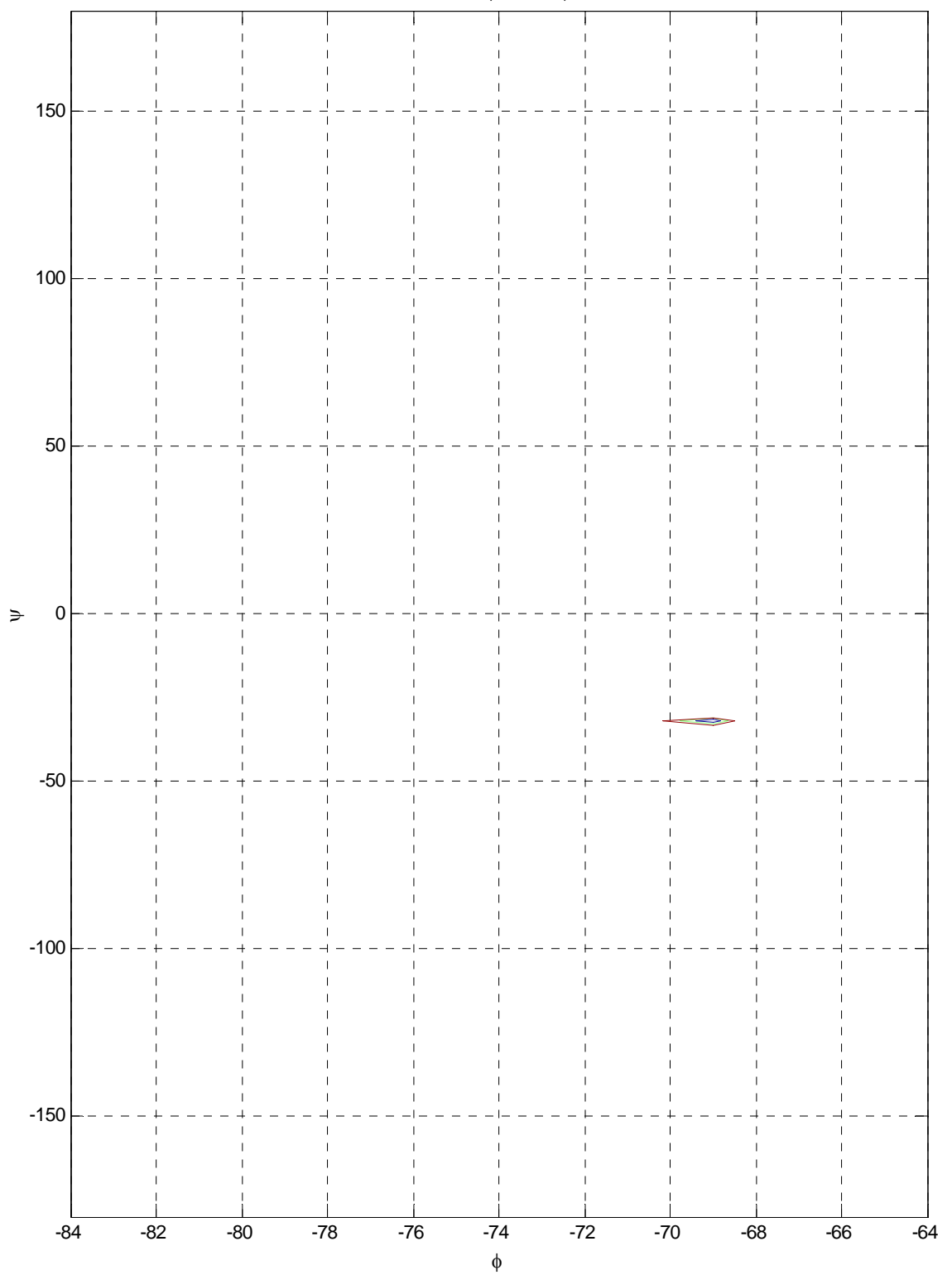

$t_{1}$ fit

Ramachandran $\chi^{2}$ map for GAV with step of 5 degrees Minimum at: $\phi=-69, \psi=-30$

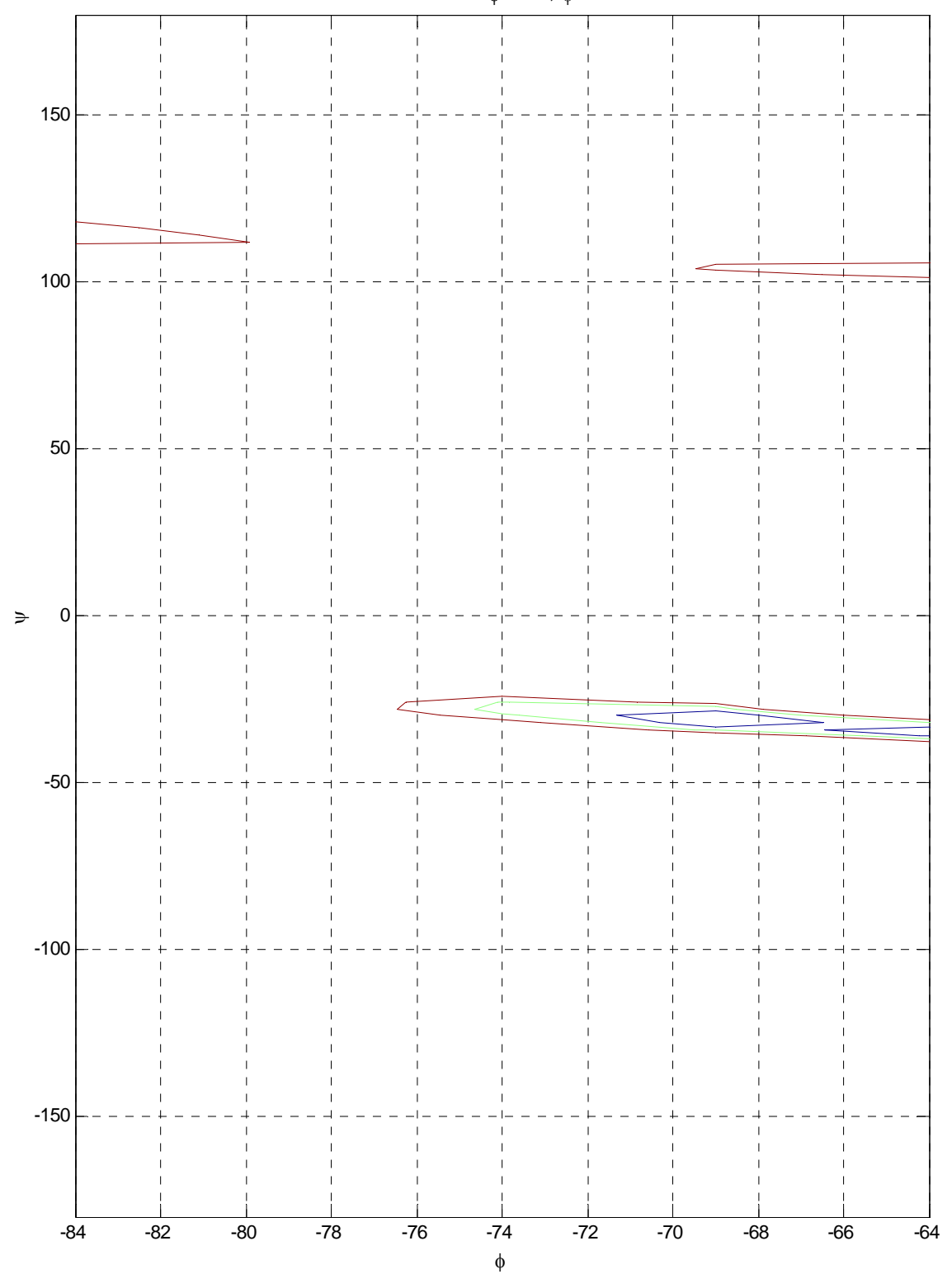




\section{5×2 degree GEN grid}

\section{Fourier Series}

Ramachandran $\chi^{2}$ map for GAV with step of 5 degrees Minimum at: $\phi=-75, \psi=-20$

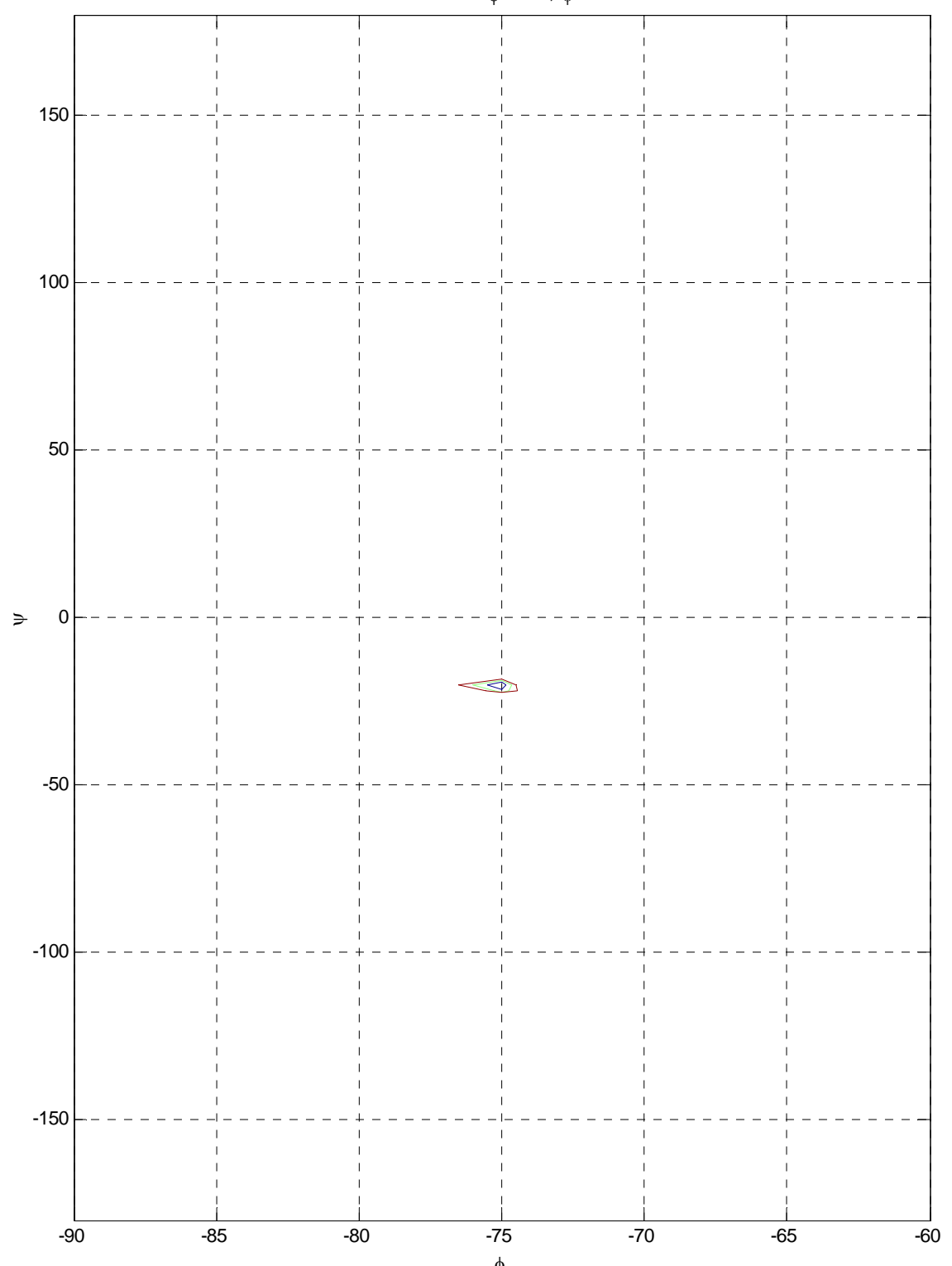

$t_{1}$ fit

Ramachandran $\chi^{2}$ map for GAV with step of 5 degrees Minimum at: $\phi=-80, \psi=110$

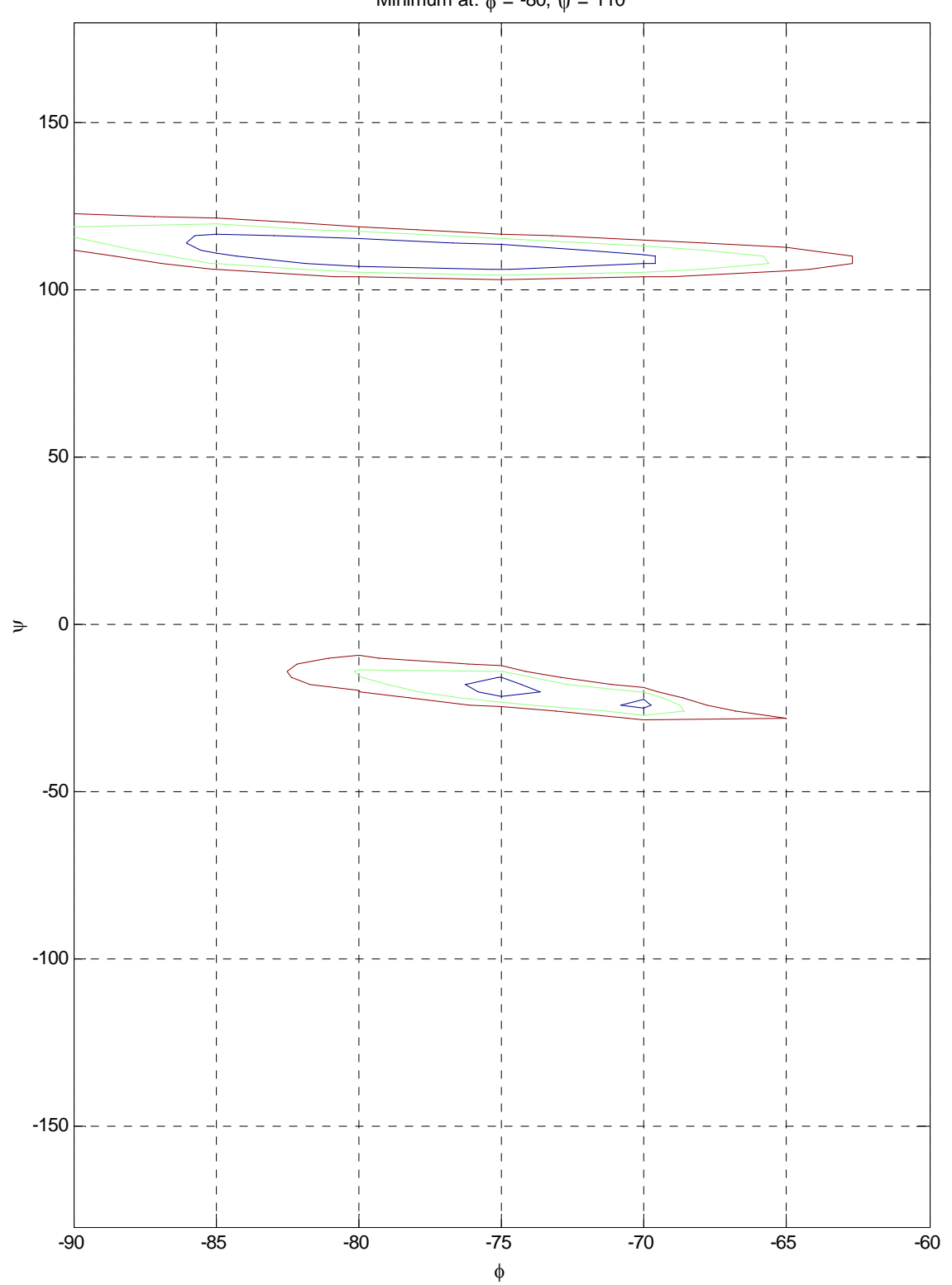




\section{5x2 degree GAV grid}

$$
-69-32 \chi^{2}=232
$$
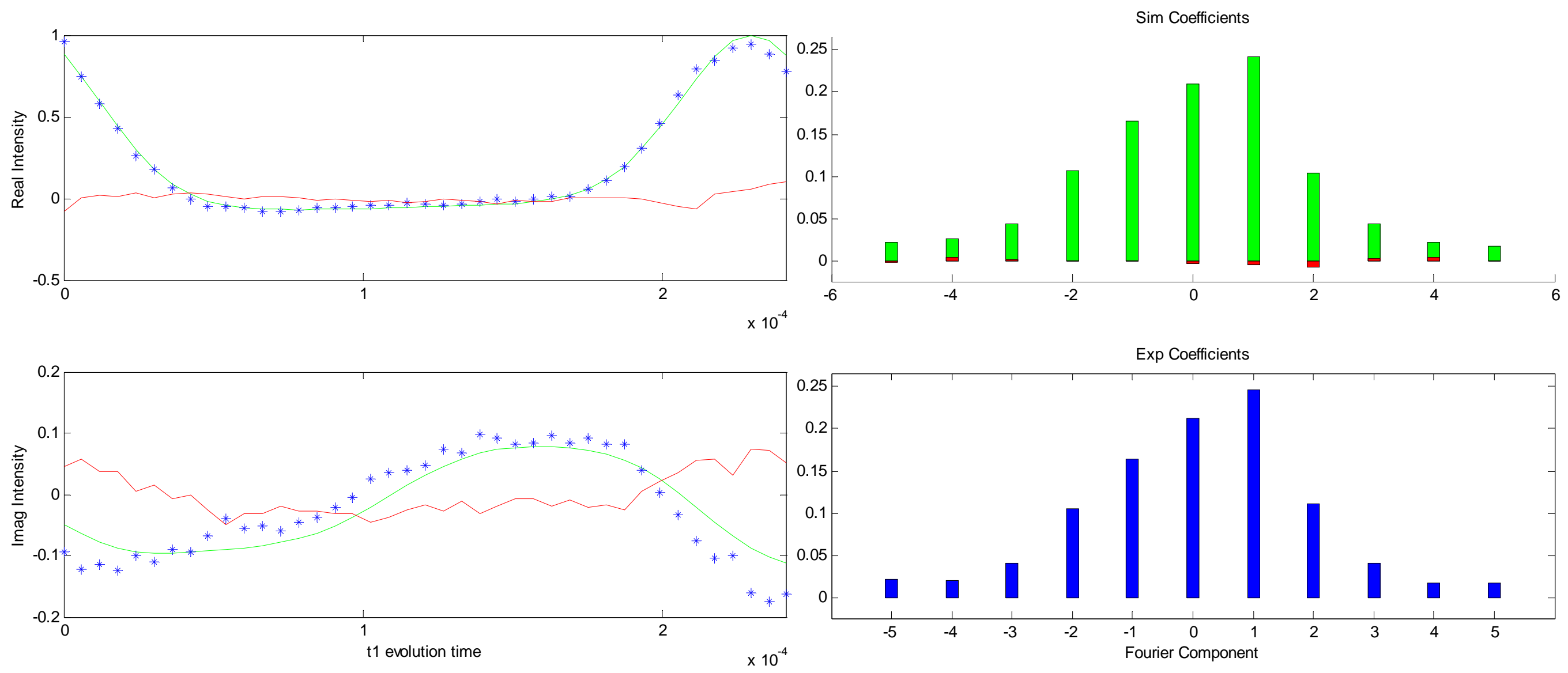

Exp Coefficients

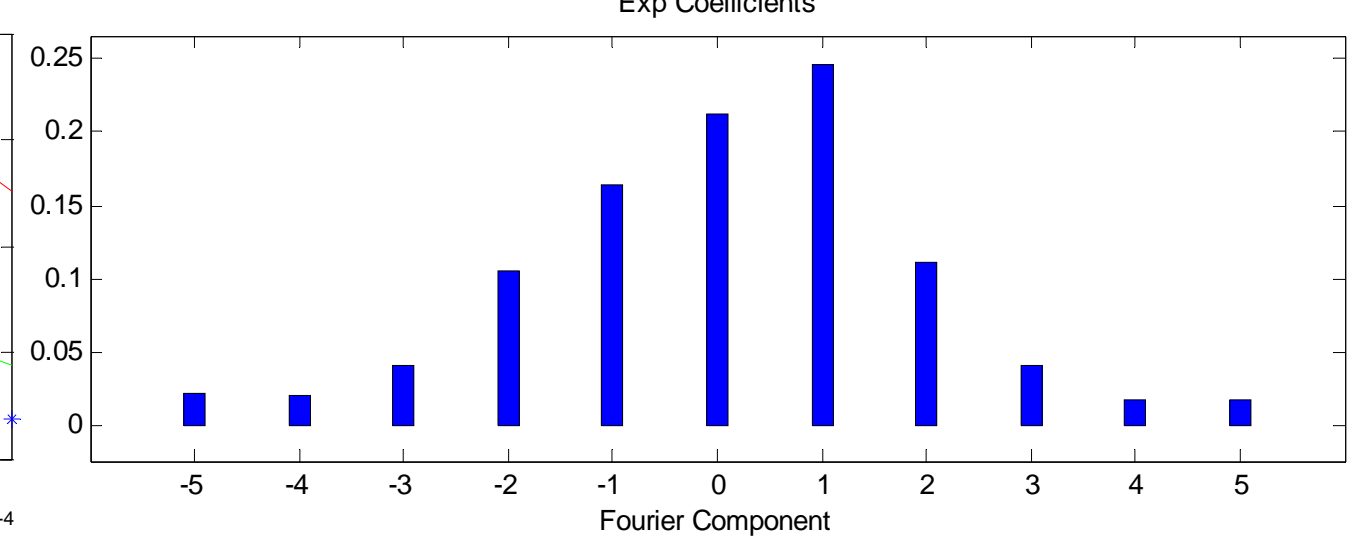




\section{0 degree GAF grid}

\section{Fourier Series}

Ramachandran $\chi^{2}$ map for GAF with step of 10 degrees Minimum at: $\phi=-80, \psi=-30$

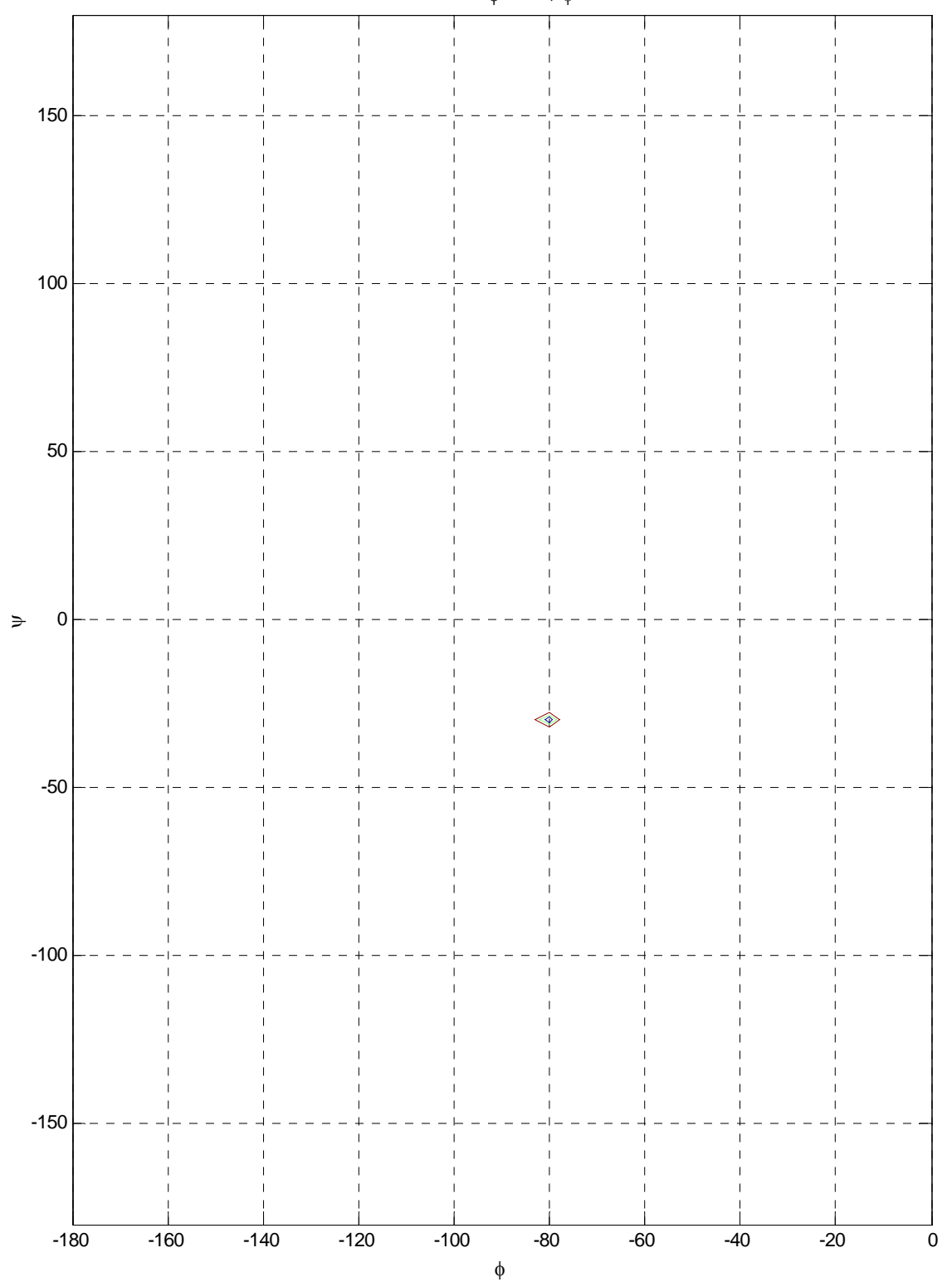

$t_{1}$ fit

Ramachandran $\chi^{2}$ map for GAF with step of 10 degrees Minimum at: $\phi=-90, \psi=-30$

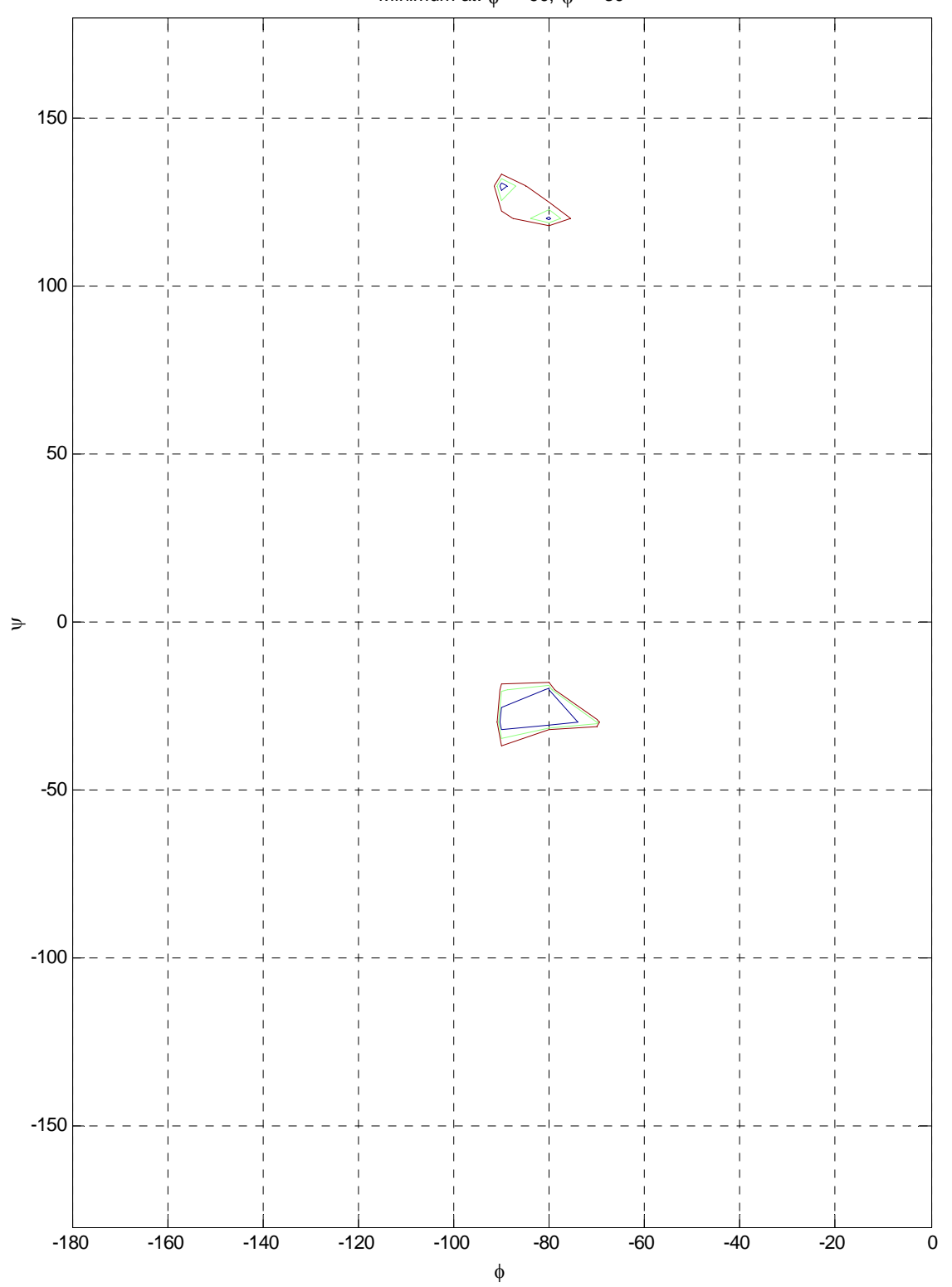




\section{X2 degree GAF grid}

\section{Fourier Series}

Ramachandran $\chi^{2}$ map for GAF with step of 5 degrees Minimum at: $\phi=-83, \psi=-30$

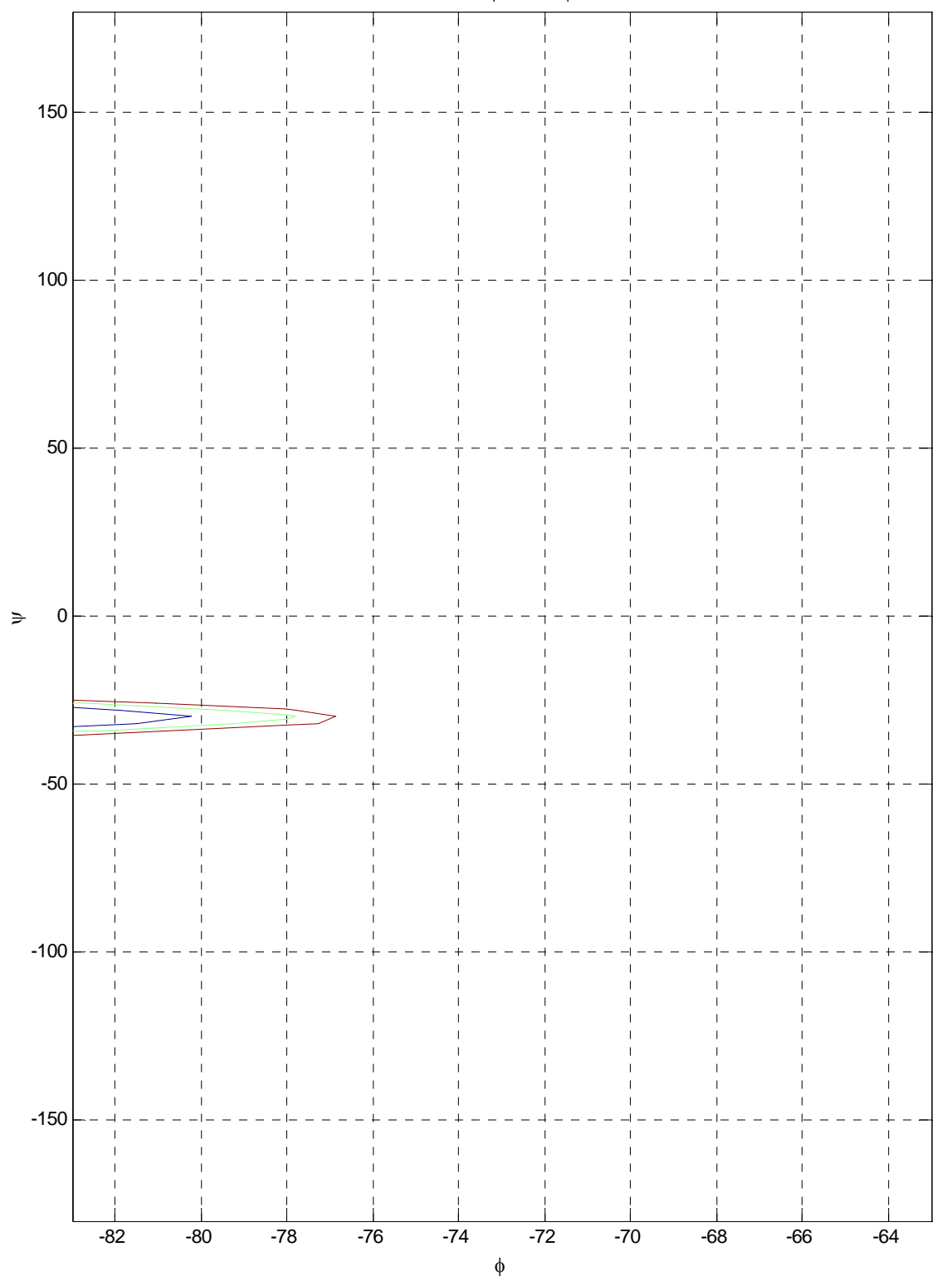

$t_{1}$ fit

Ramachandran $\chi^{2}$ map for GAF with step of 5 degrees Minimum at: $\phi=-83, \psi=-26$

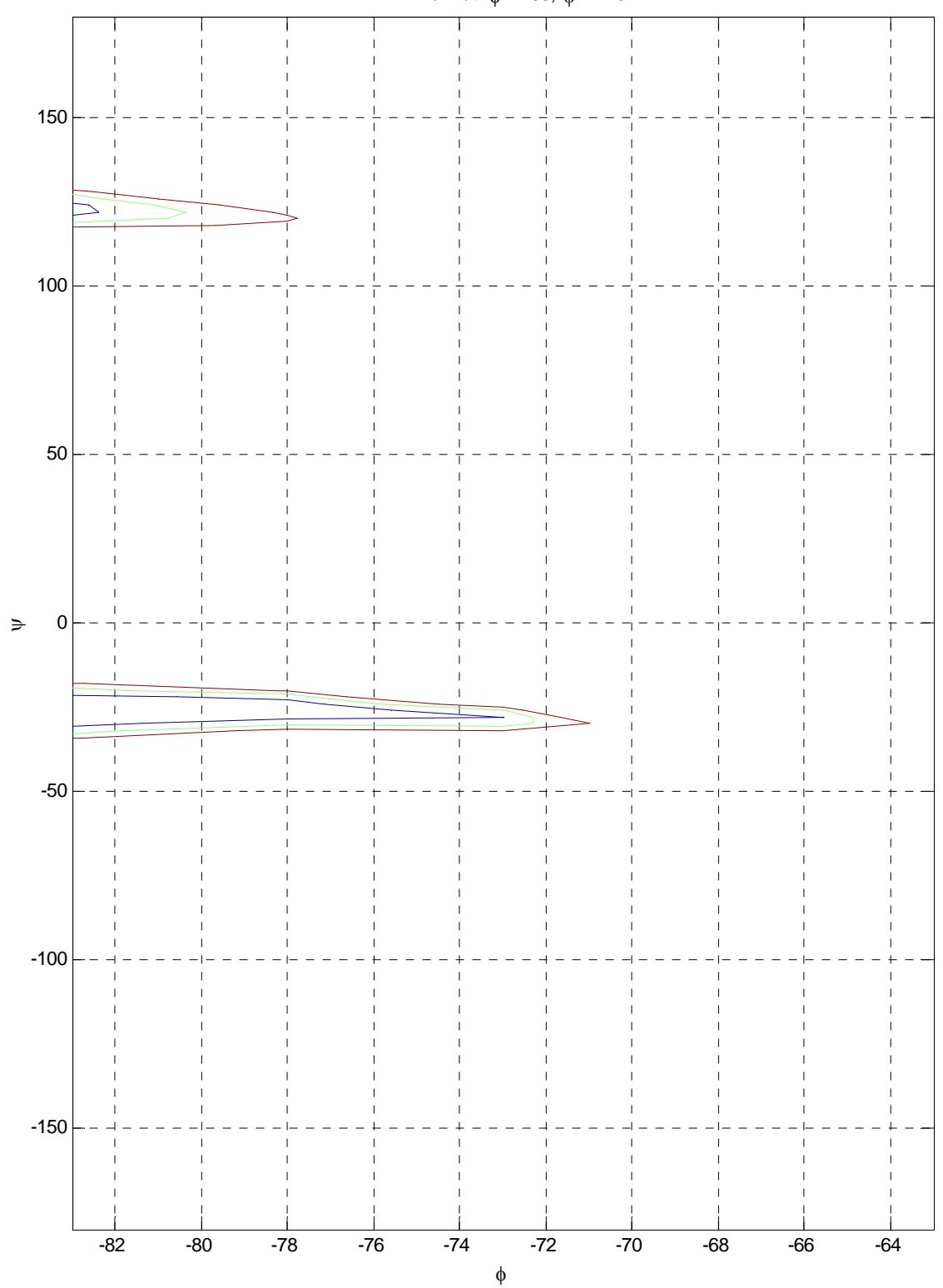




\section{5×2 degree GEN grid}

\section{Fourier Series}

Ramachandran $\chi^{2}$ map for GAF with step of 5 degrees Minimum at: $\phi=-85, \psi=-20$

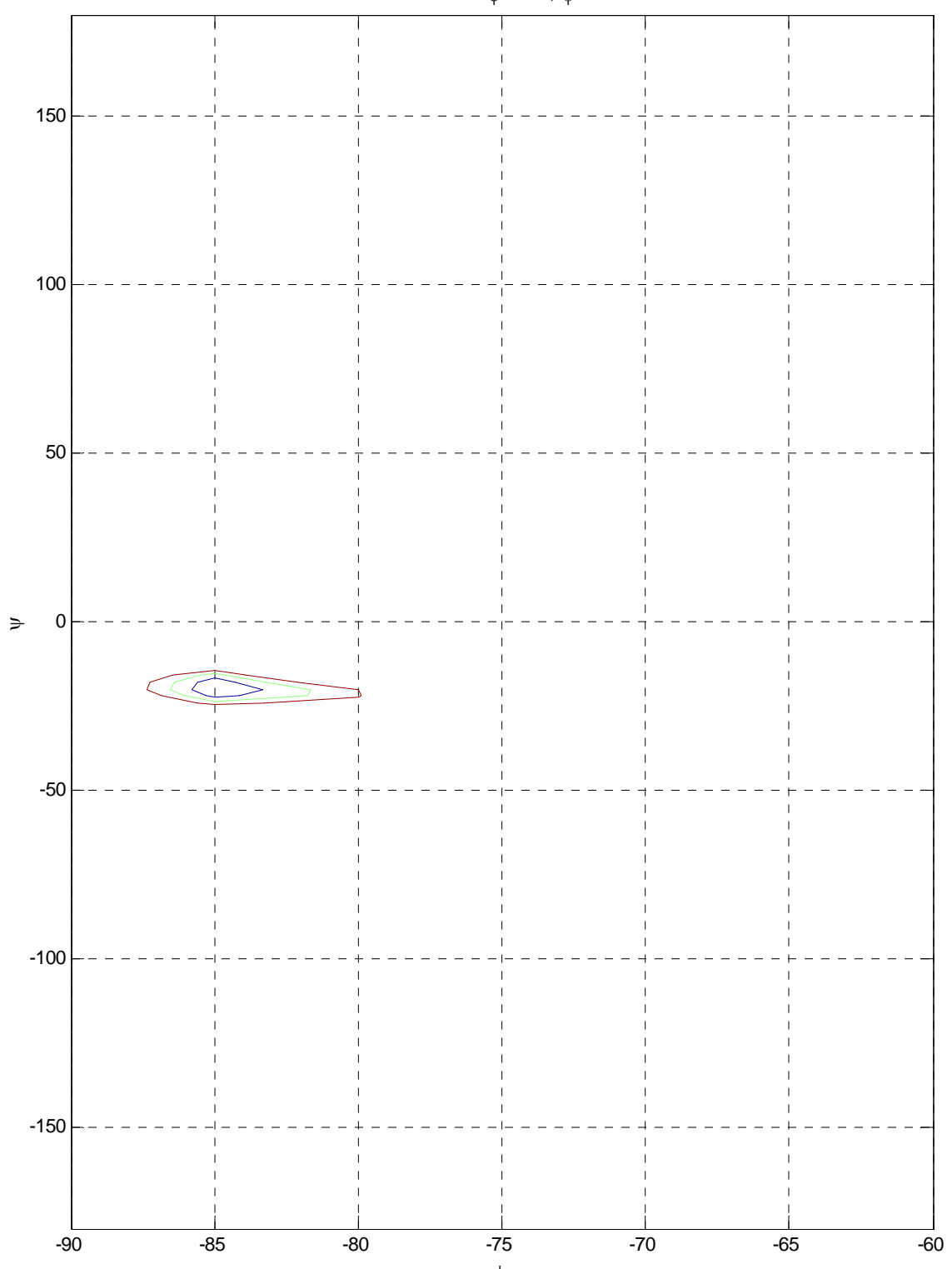

$t_{1}$ fit

Ramachandran $\chi^{2}$ map for GAF with step of 5 degrees Minimum at: $\phi=-85, \psi=122$

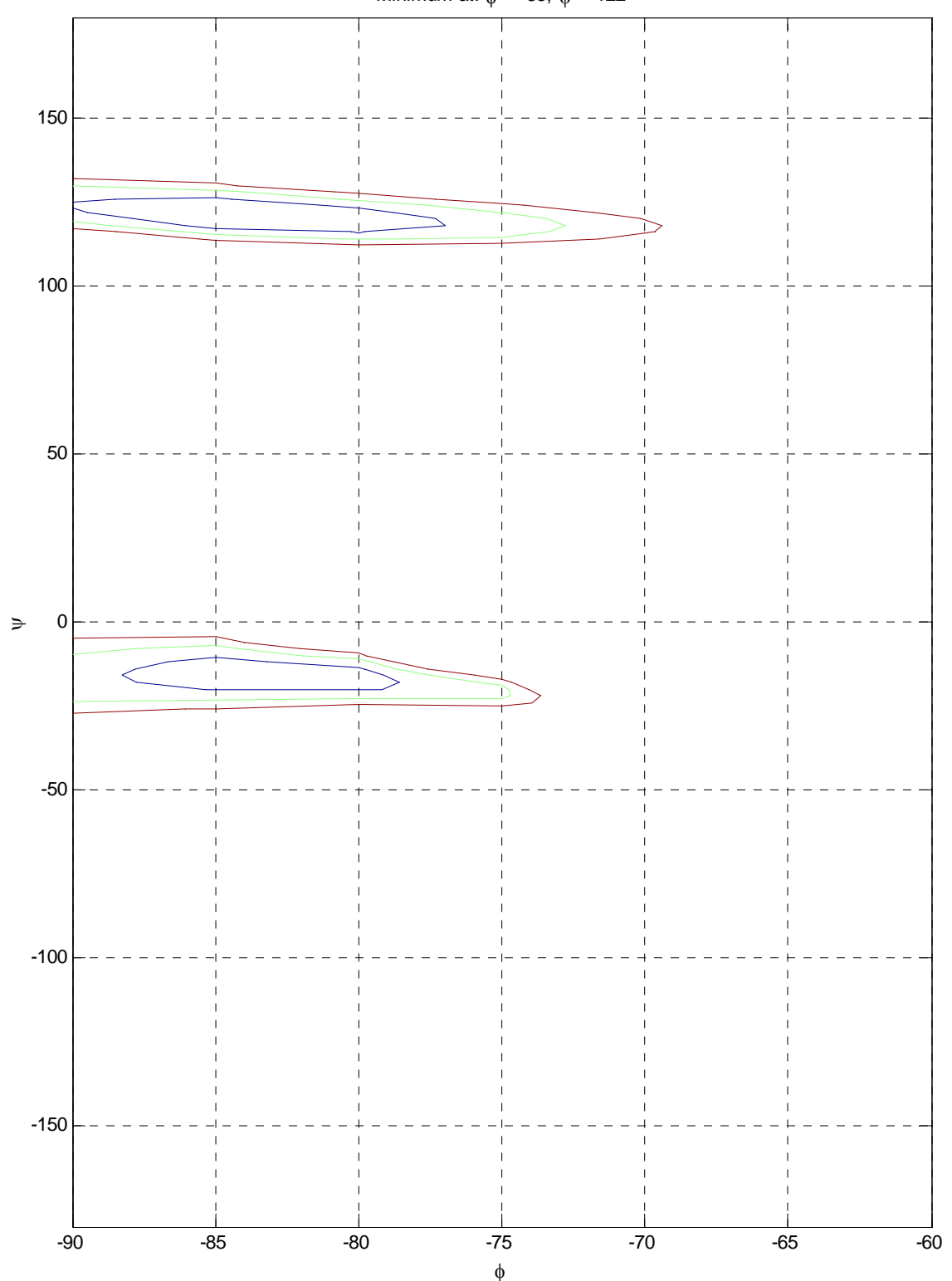




\section{0 degree GGV grid}

\section{Fourier Series}

Ramachandran $\chi^{2}$ map for GGV with step of 10 degrees Minimum at: $\phi=-80, \psi=-30$

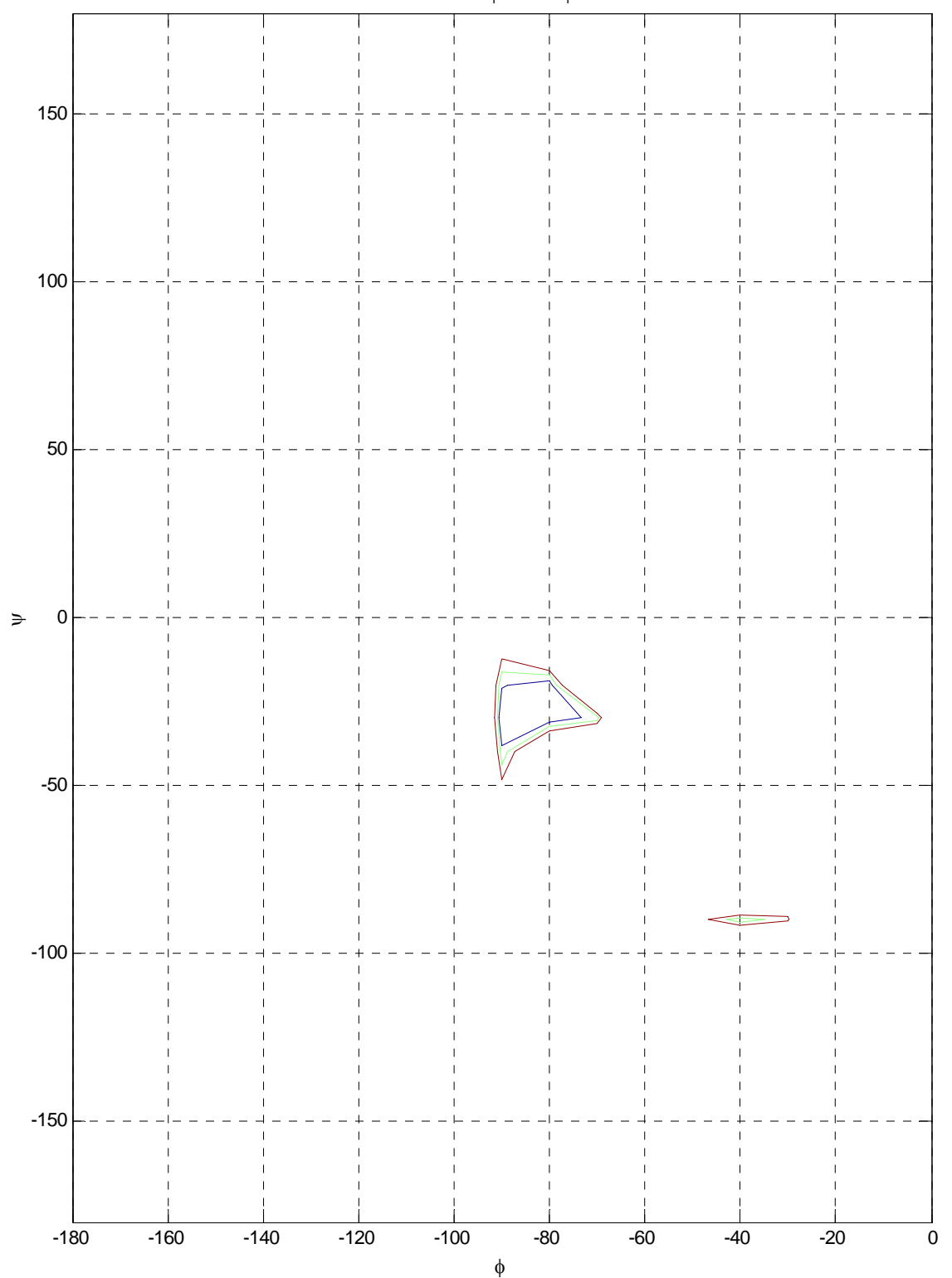

$t_{1}$ fit

Ramachandran $\chi^{2}$ map for GGV with step of 10 degrees Minimum at: $\phi=-90, \psi=120$

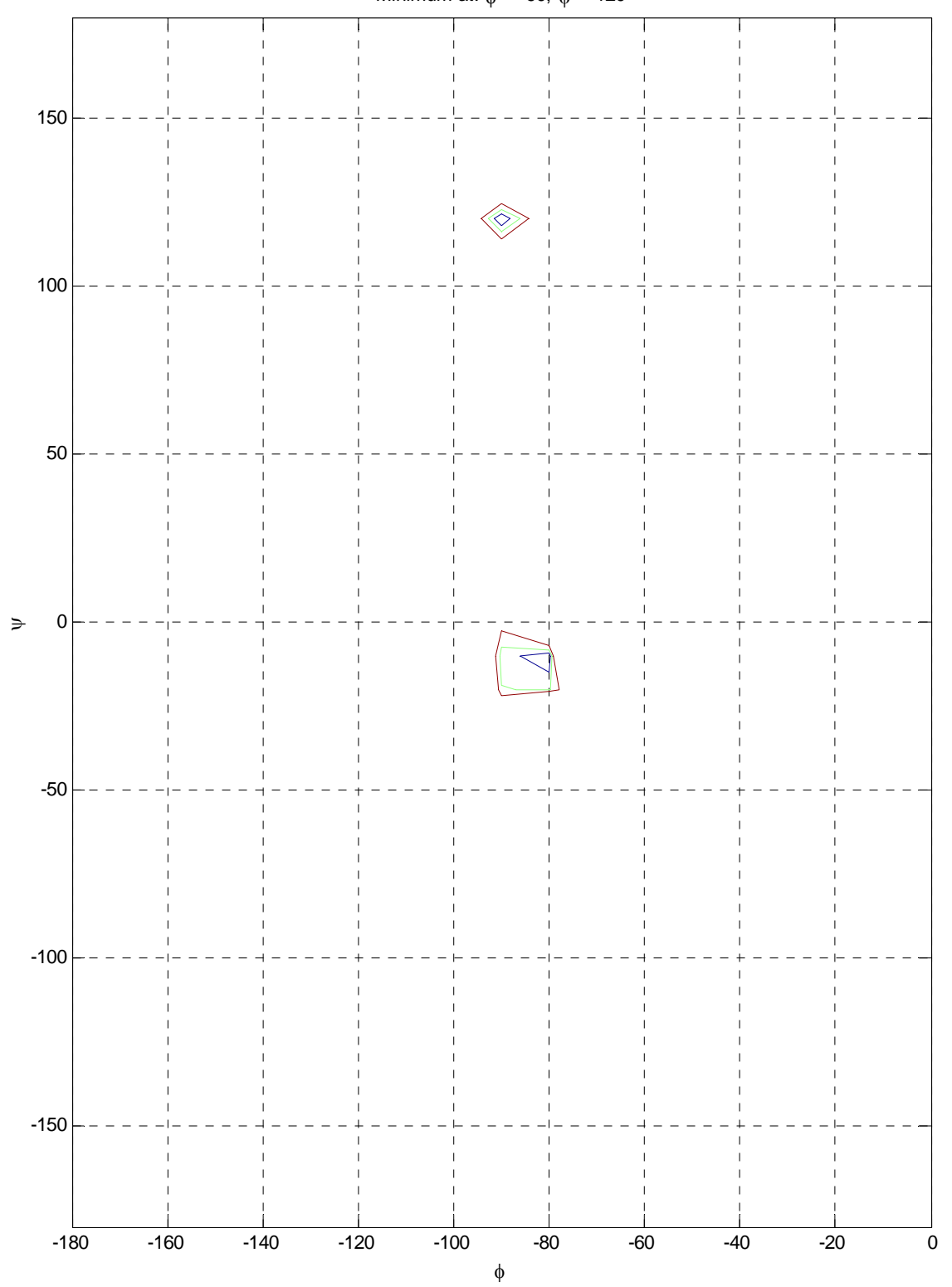




\section{5×2 degree GGV grid}

\section{Fourier Series}

Ramachandran $\chi^{2}$ map for GGV with step of 5 degrees Minimum at: $\phi=-82, \psi=-26$

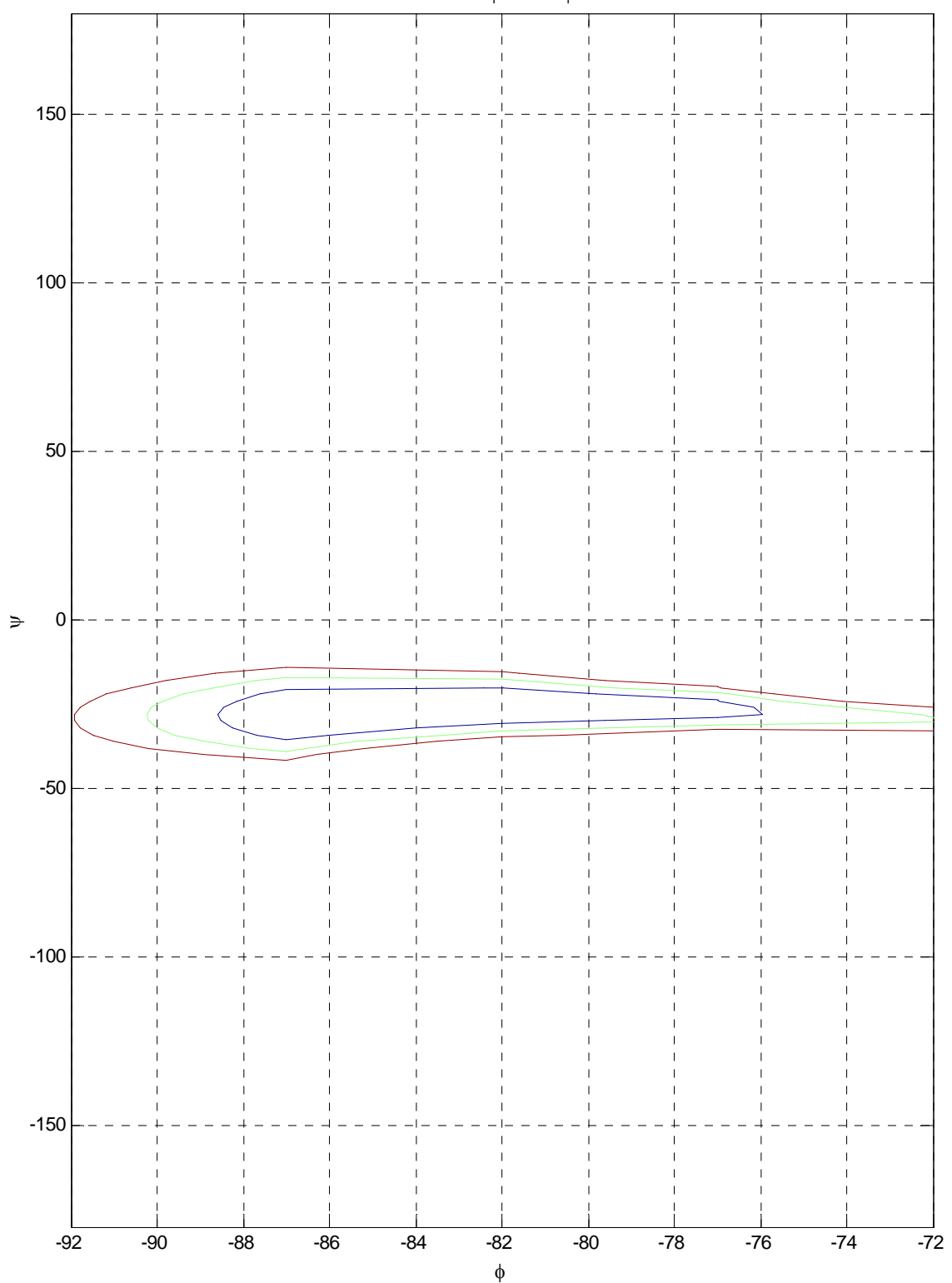

$t_{1}$ fit

Ramachandran $\chi^{2}$ map for GGV with step of 5 degrees Minimum at: $\phi=-82, \psi=-12$

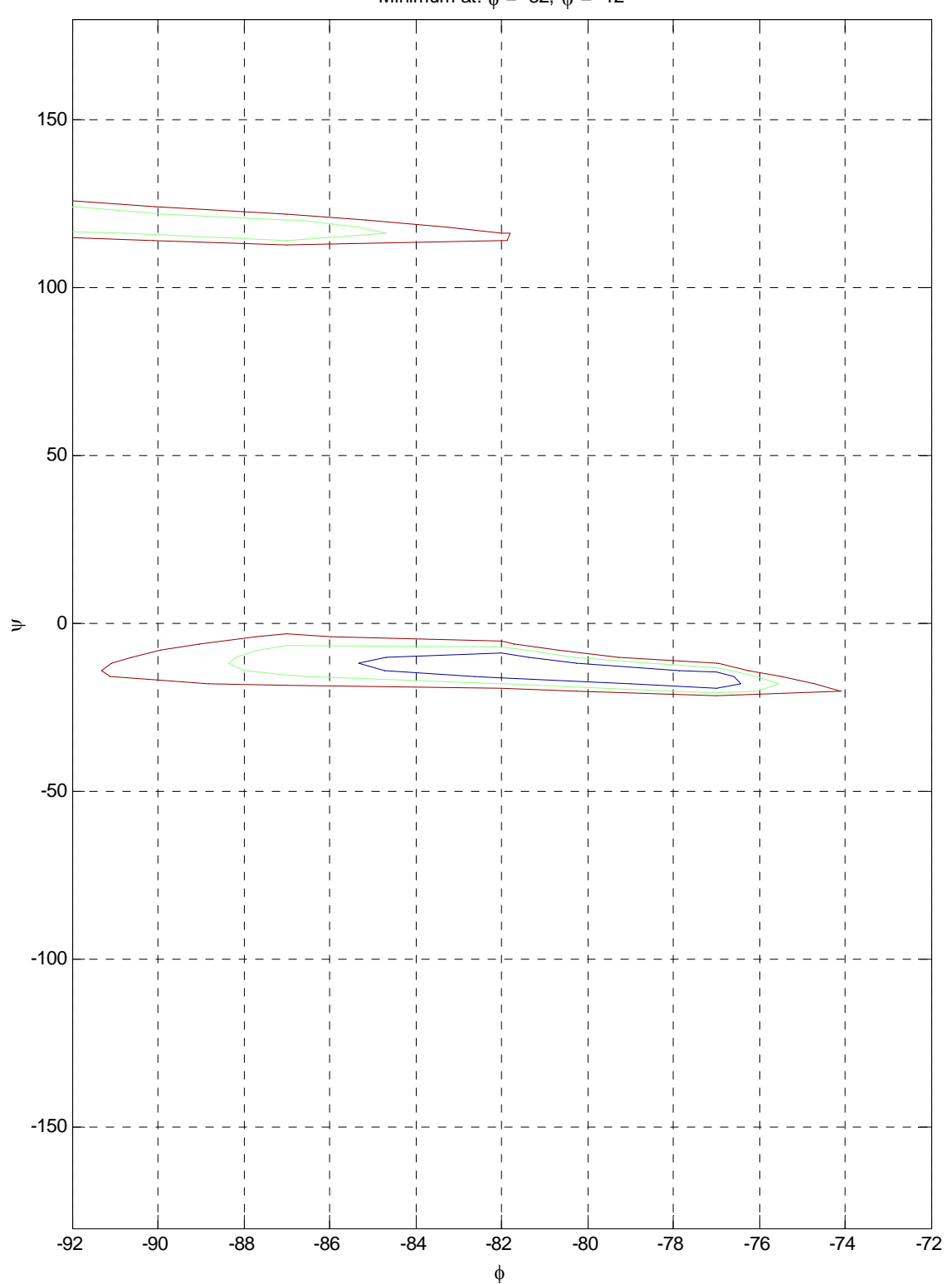




\section{5×2 degree GEN grid}

\section{Fourier Series}

Ramachandran $\chi^{2}$ map for GGV with step of 5 degrees Minimum at: $\phi=-85, \psi=-26$

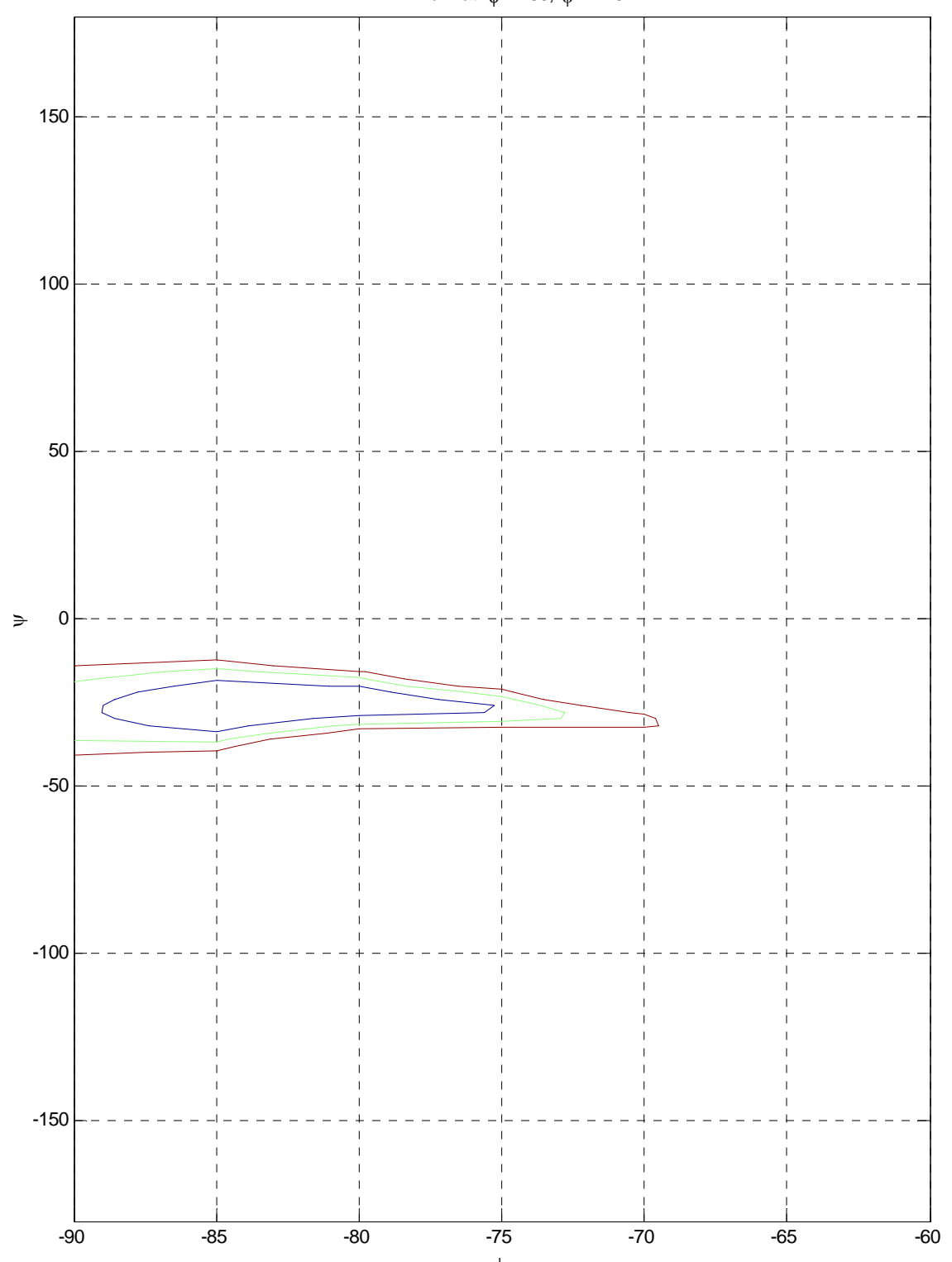

$t_{1}$ fit

Ramachandran $\chi^{2}$ map for GGV with step of 5 degrees Minimum at: $\phi=-80, \psi=-12$

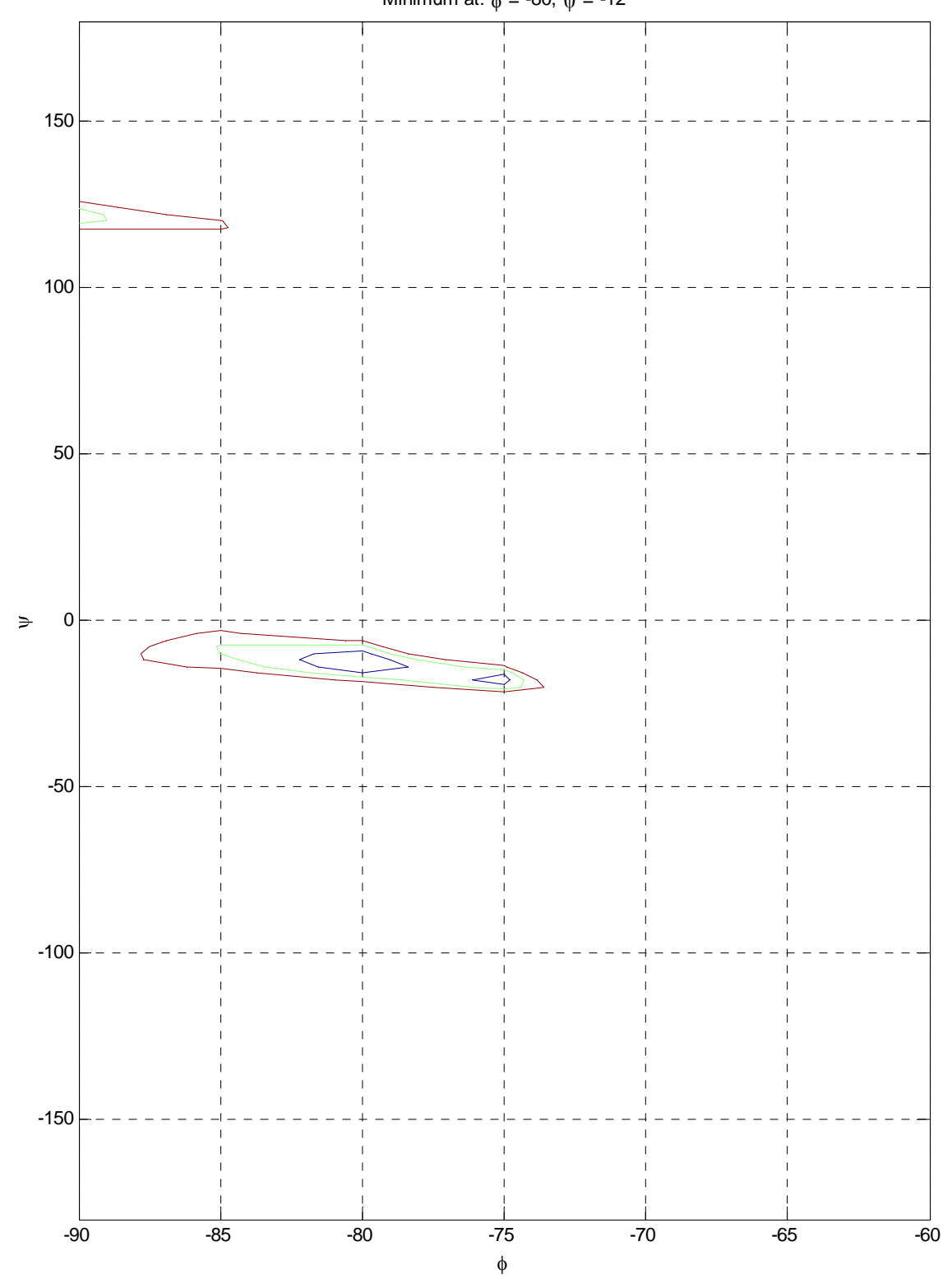




\section{0 degree AGG grid}

\section{Fourier Series}

Ramachandran $\chi^{2}$ map for AGG with step of 10 degrees Minimum at: $\phi=-80, \psi=150$

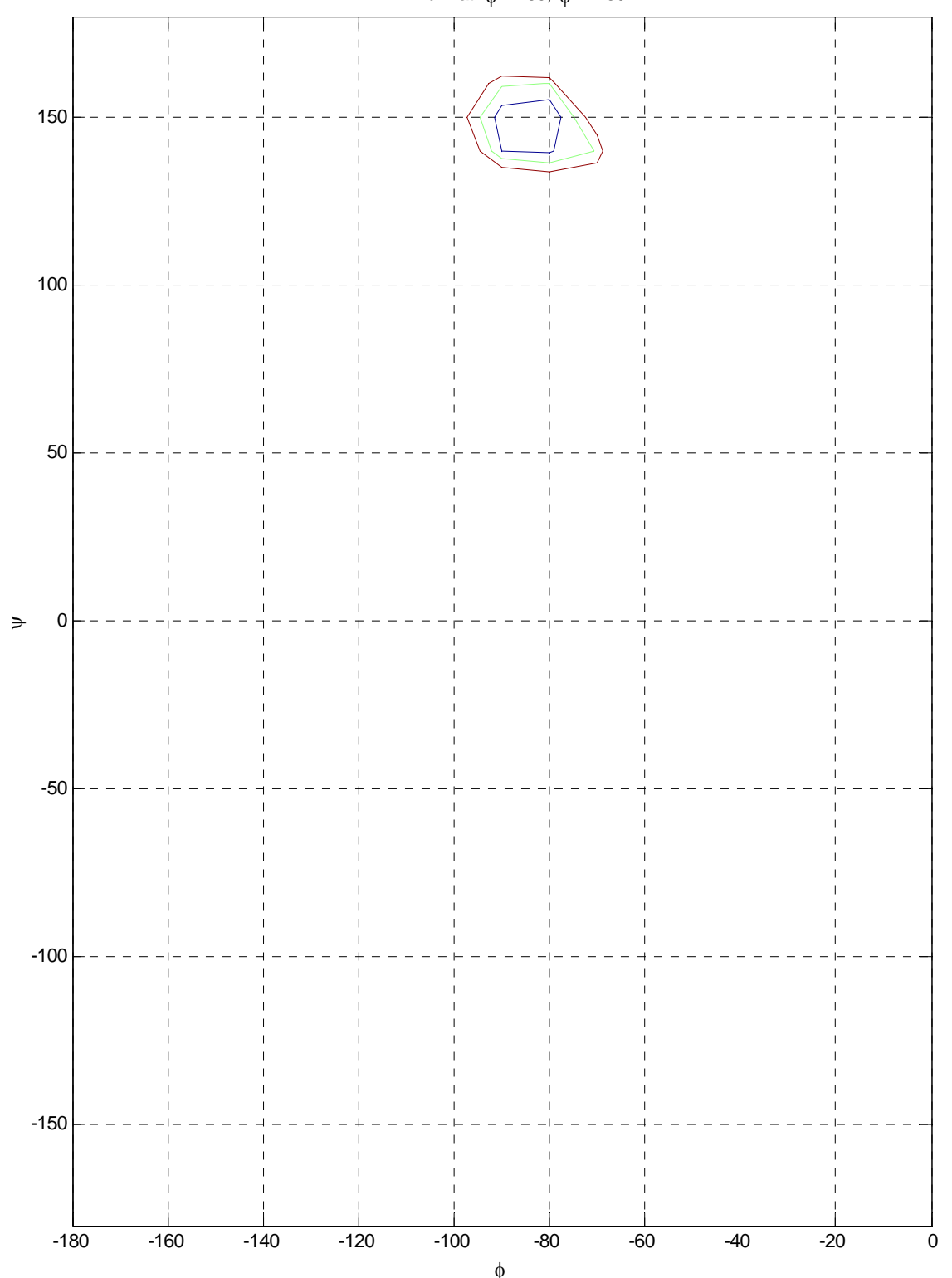

$t_{1}$ fit

Ramachandran $\chi^{2}$ map for AGG with step of 10 degrees Minimum at: $\phi=-70, \psi=150$

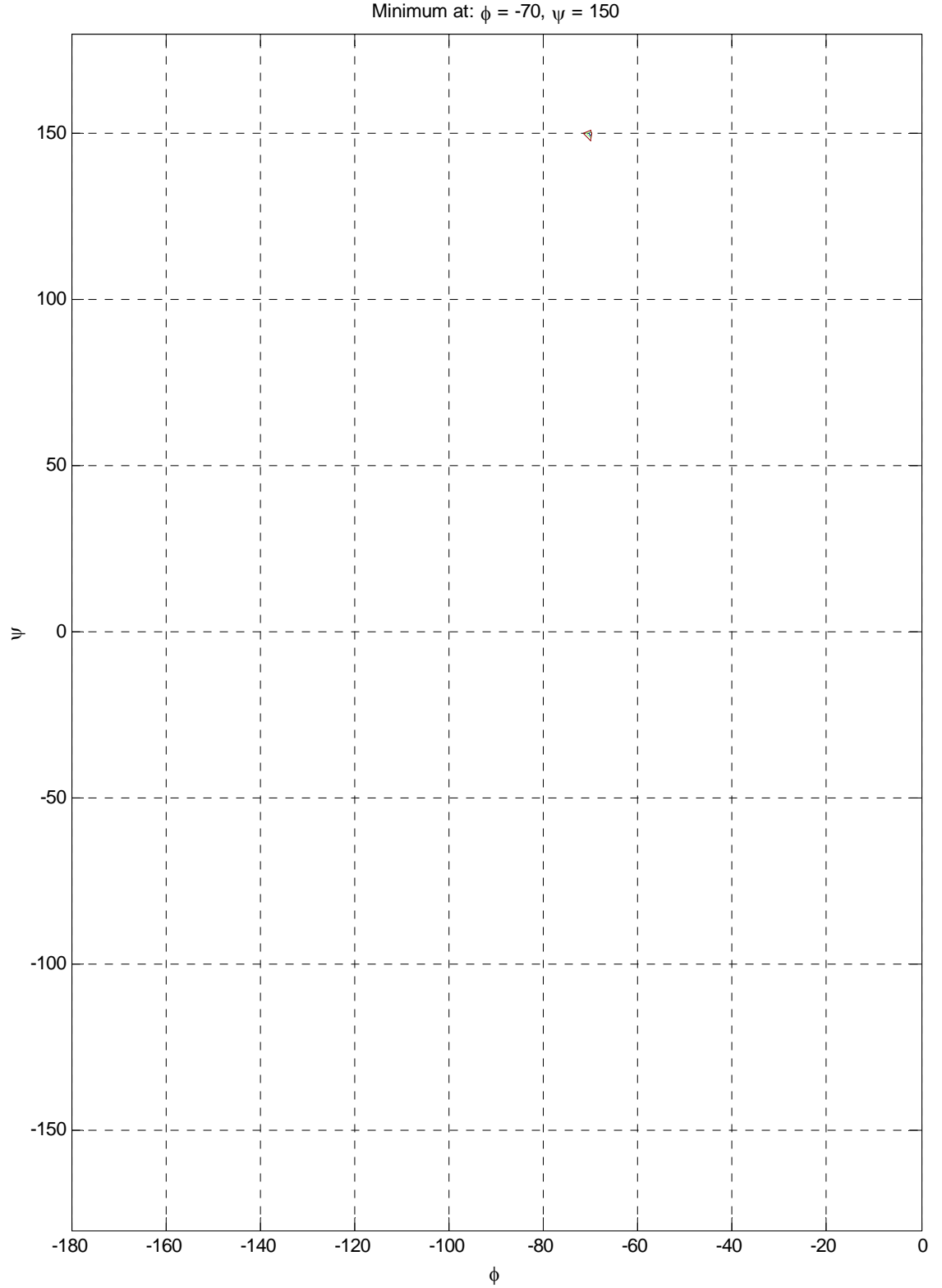


$5 \times 2$ degree $A G G$ grid

\section{Fourier Series}

Ramachandran $\chi^{2}$ map for AGG with step of 5 degrees Minimum at: $\phi=-83, \psi=148$

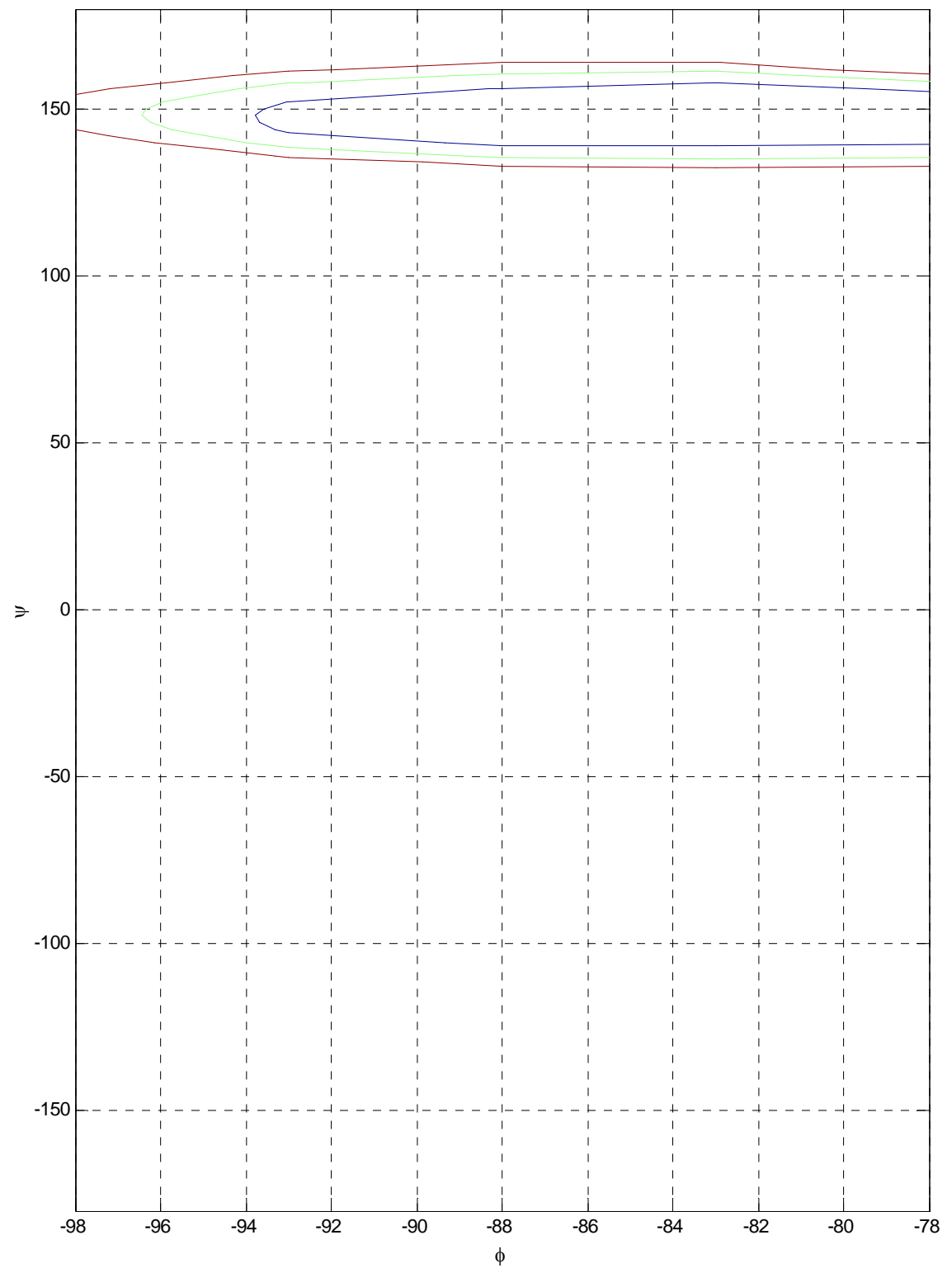

$t_{1}$ fit

Ramachandran $\chi^{2}$ map for AGG with step of 5 degrees Minimum at: $\phi=-78, \psi=158$

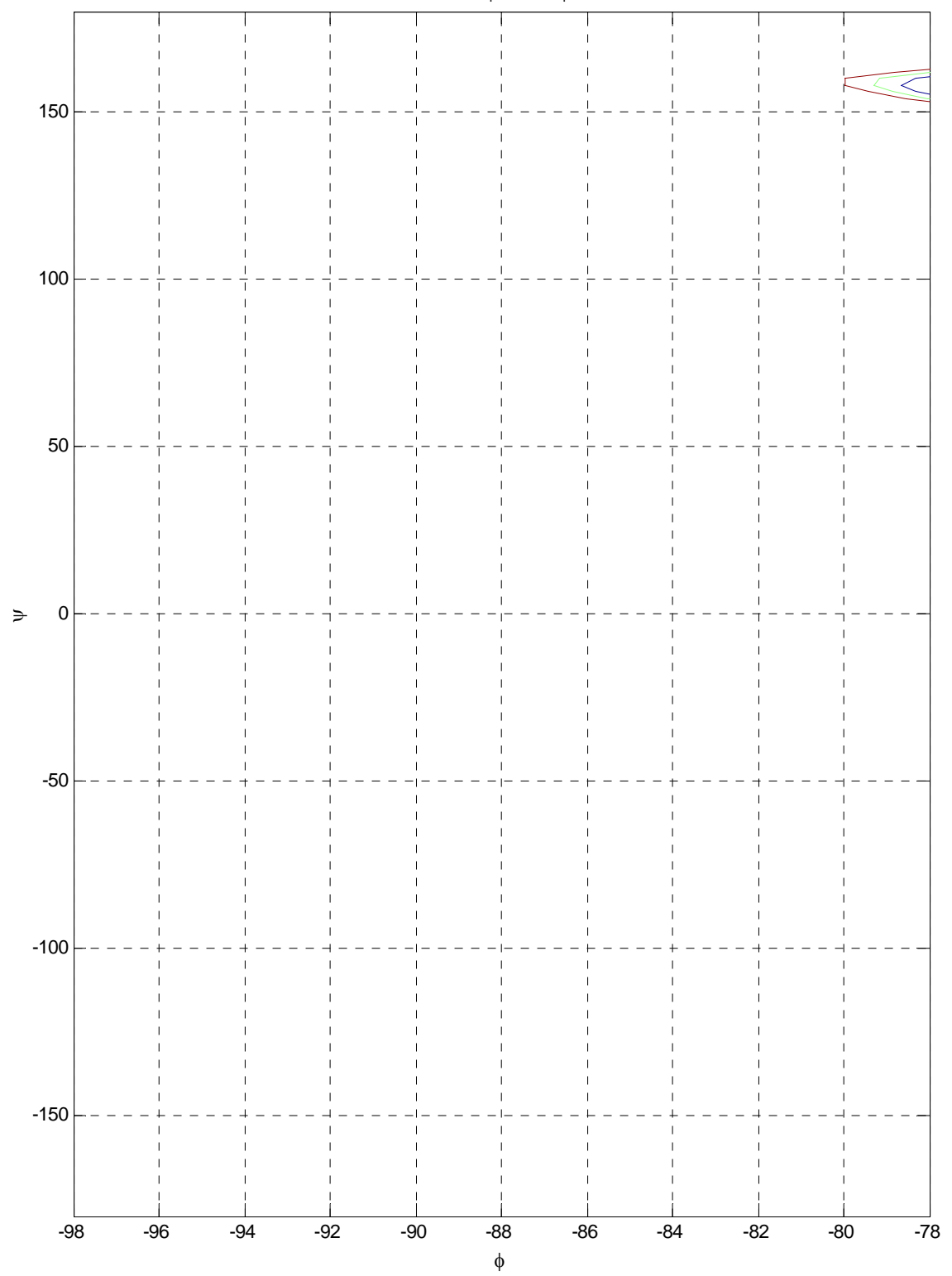




\section{5x2 degree GEN grid}

\section{Fourier Series}

Ramachandran $\chi^{2}$ map for AGG with step of 5 degrees Minimum at: $\phi=-80, \psi=150$

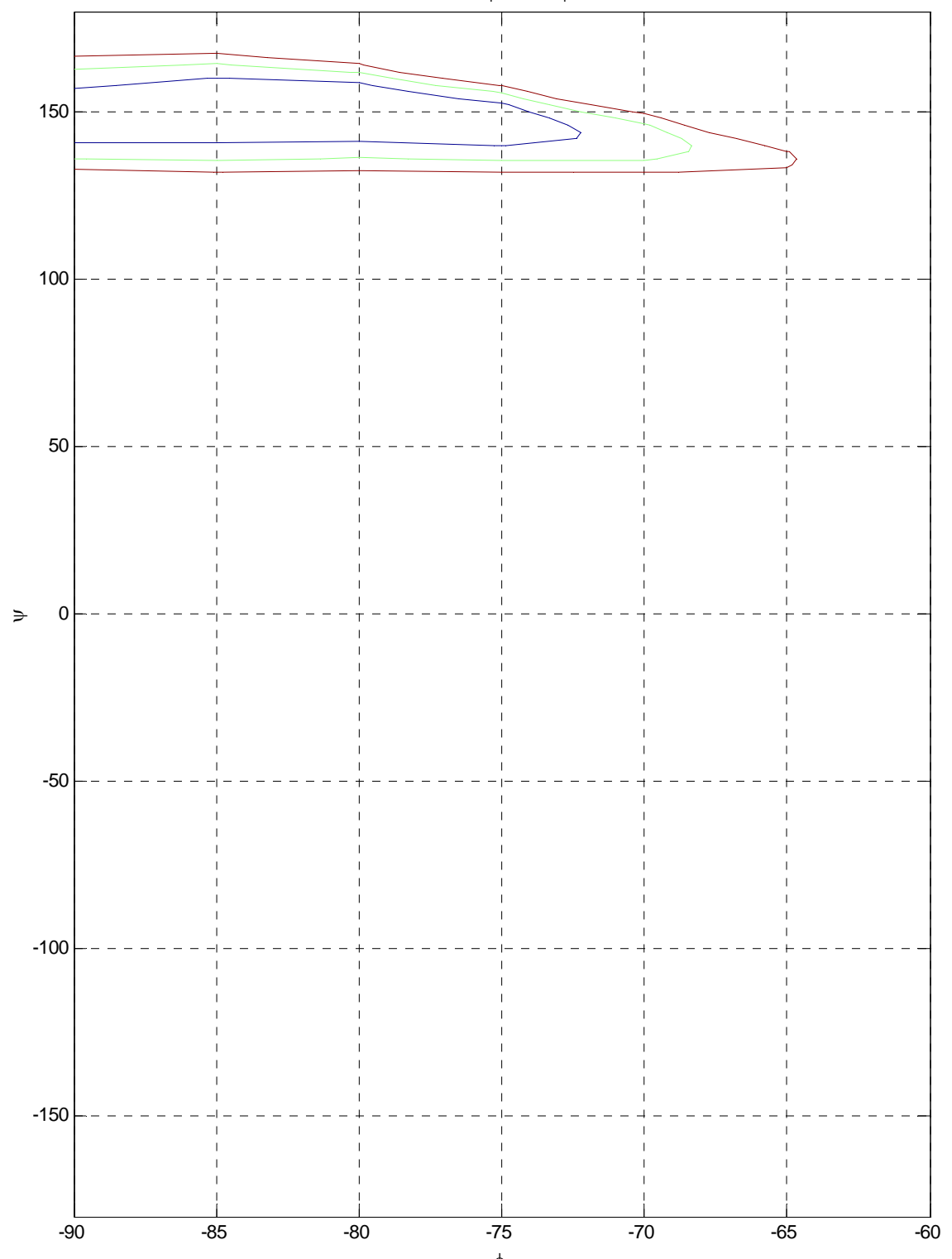

$t_{1}$ fit

Ramachandran $\chi^{2}$ map for AGG with step of 5 degrees Minimum at: $\phi=-70, \psi=150$

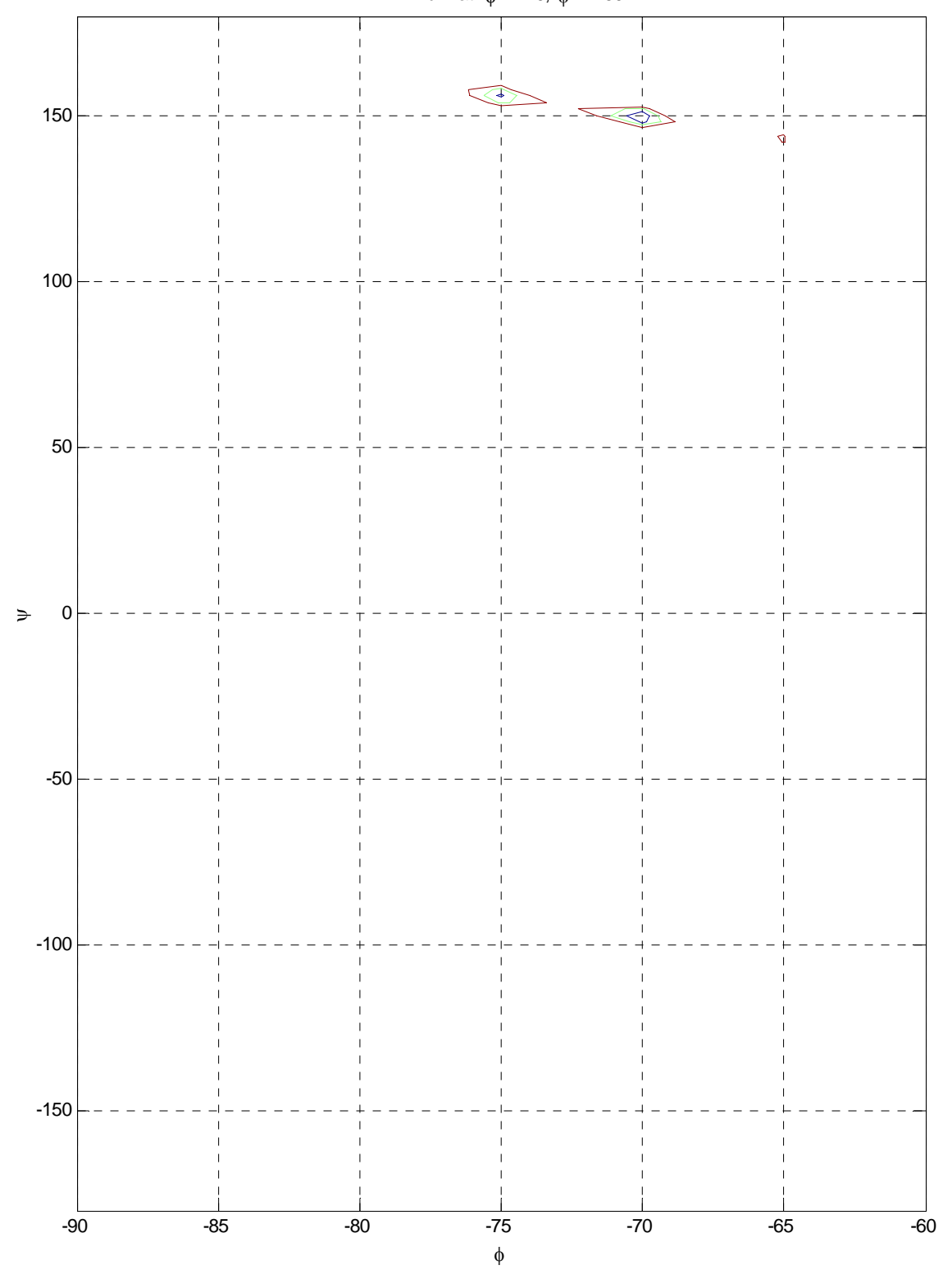




\section{0 degree FAG grid}

\section{Fourier Series}

Ramachandran $\chi^{2}$ map for FAG with step of 10 degrees Minimum at: $\phi=-100, \psi=90$

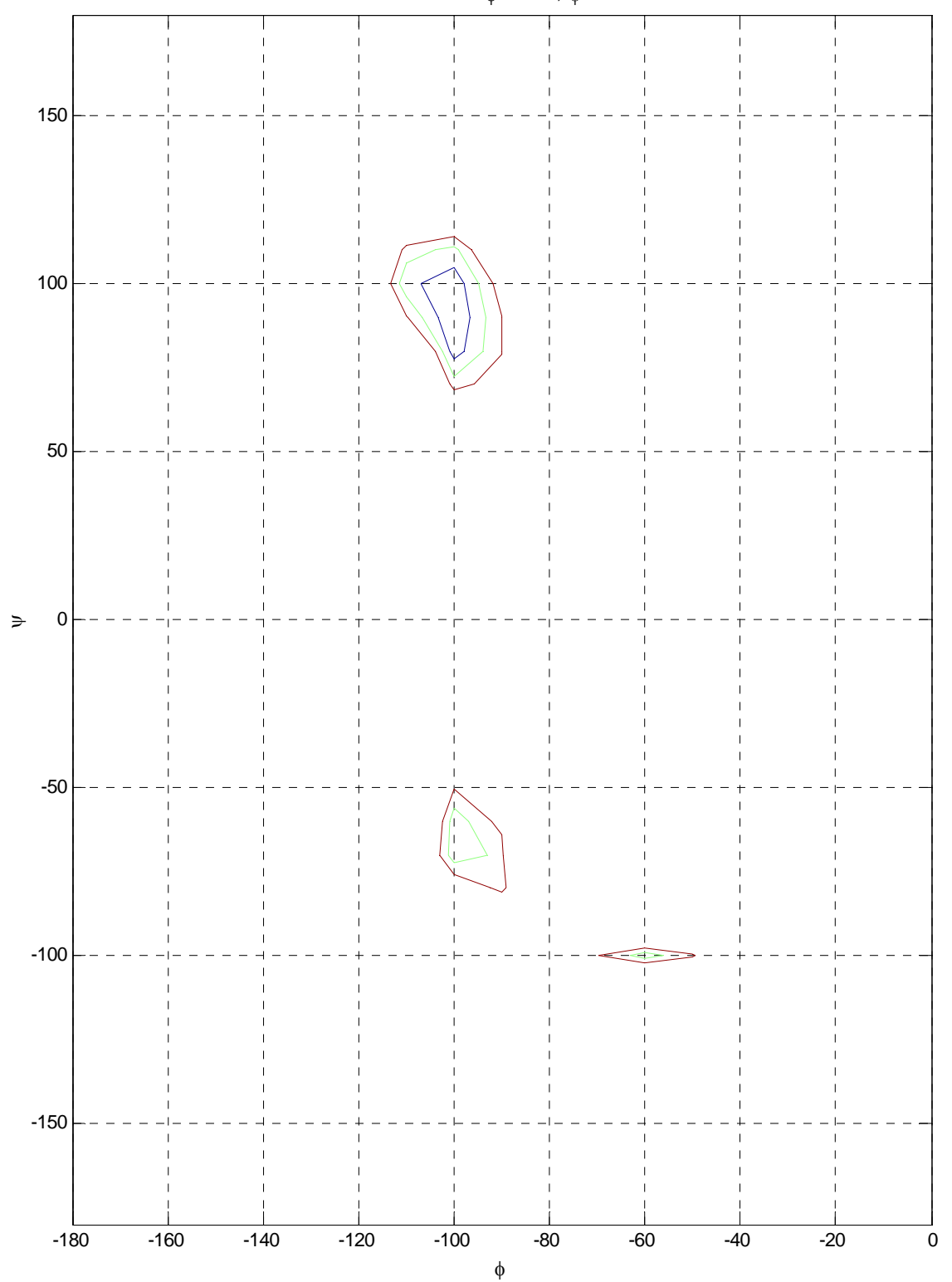

$t_{1}$ fit

Ramachandran $\chi^{2}$ map for FAG with step of 10 degrees Minimum at: $\phi=-100, \psi=-70$

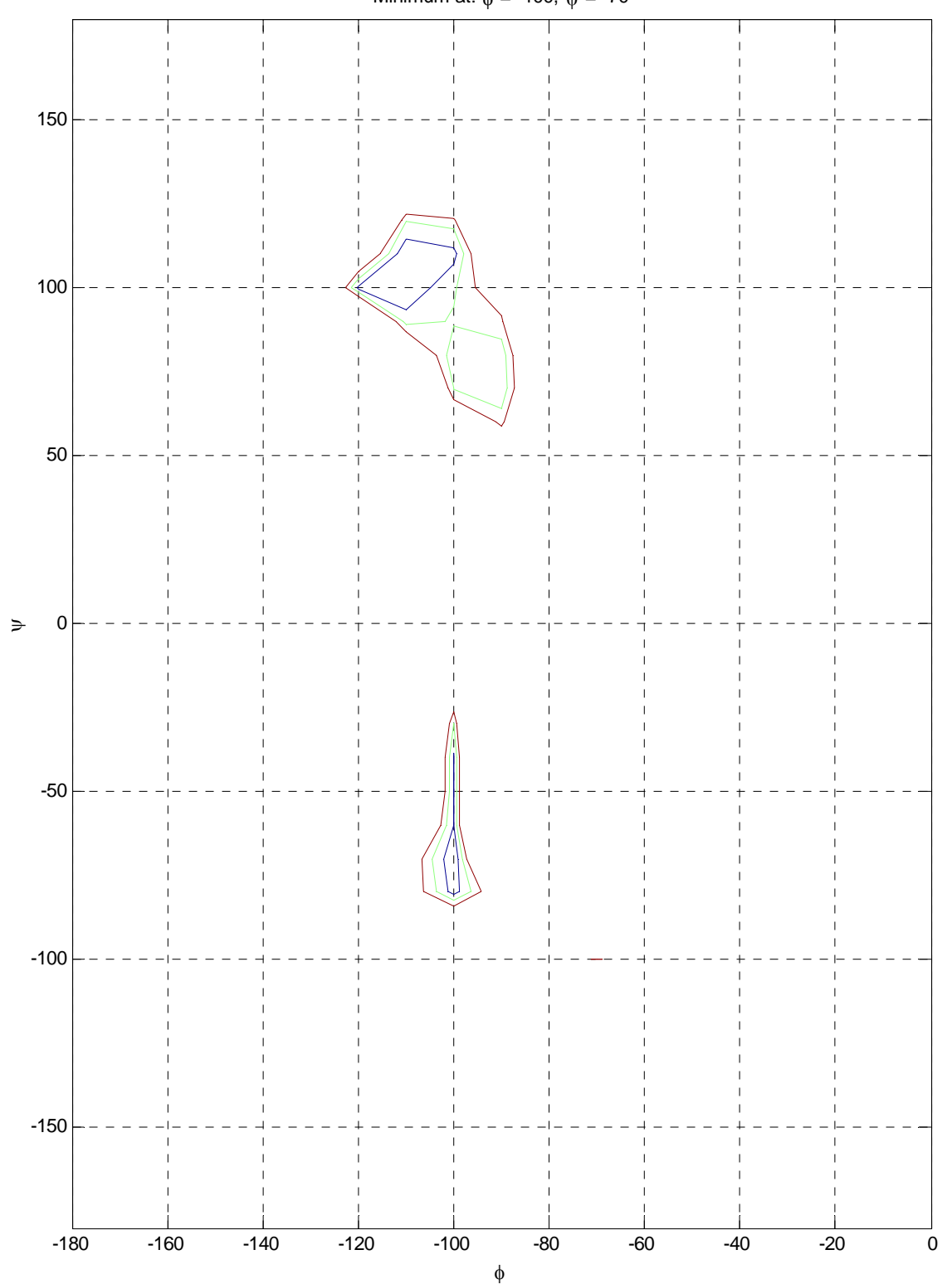




\section{$5 \times 2$ degree FAG grid}

\section{Fourier Series}

Ramachandran $\chi^{2}$ map for FAG with step of 5 degrees Minimum at: $\phi=-103, \psi=96$

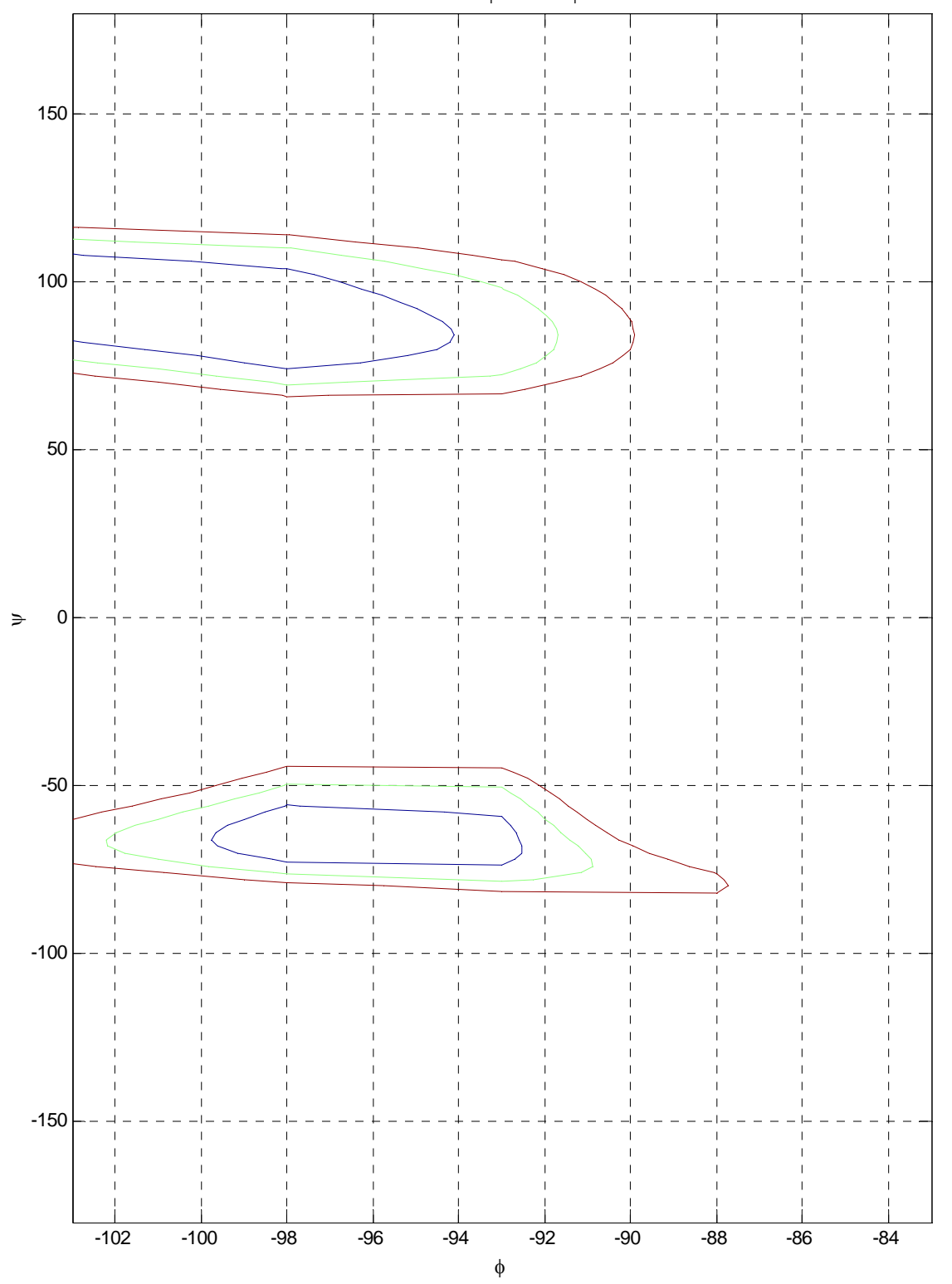

$t_{1}$ fit

Ramachandran $\chi^{2}$ map for FAG with step of 5 degrees Minimum at: $\phi=-103, \psi=112$

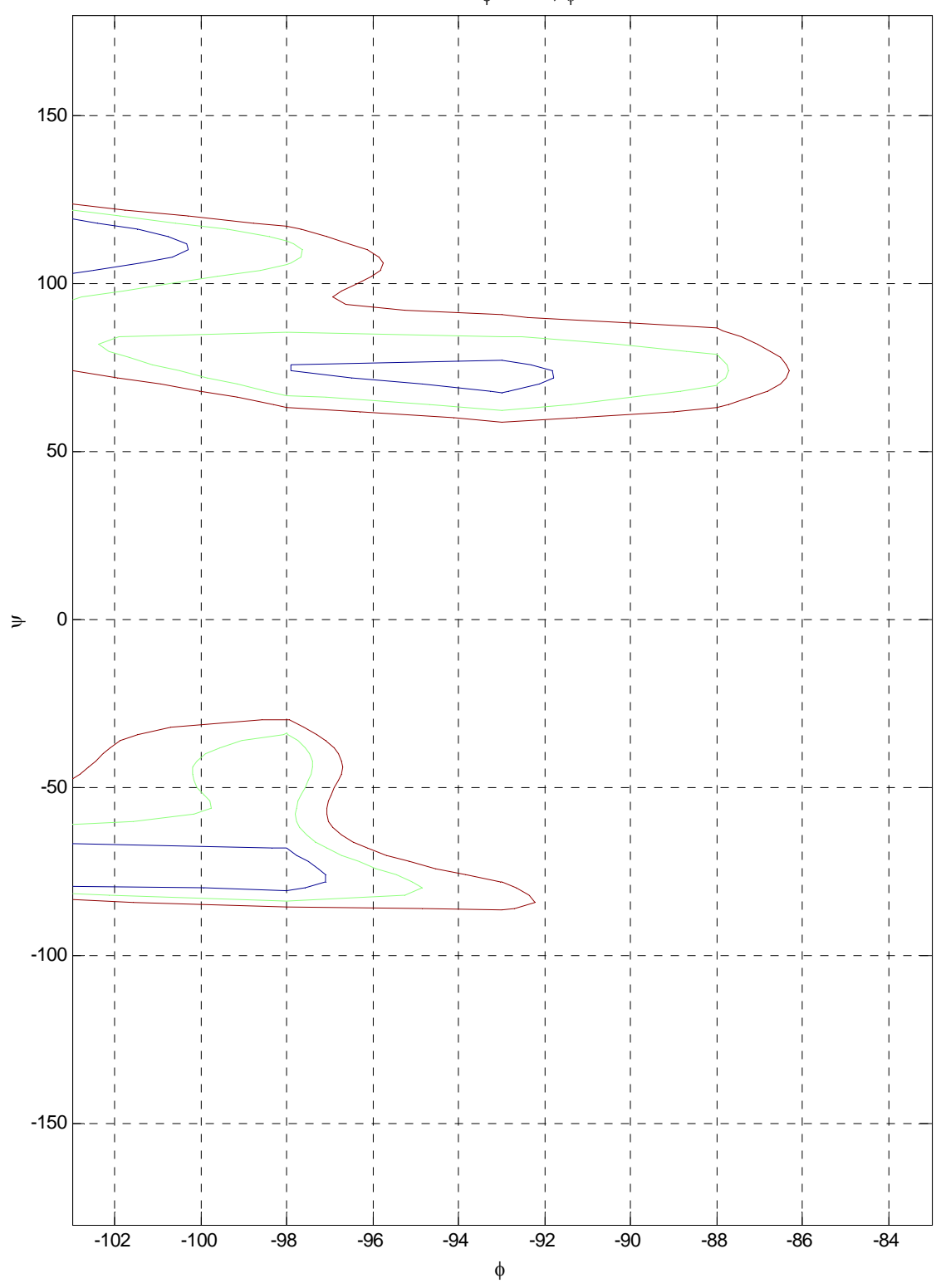




\section{5x2 degree GEN grid}

\section{Fourier Series}

Ramachandran $\chi^{2}$ map for FAG with step of 5 degrees Minimum at: $\phi=-100, \psi=86$

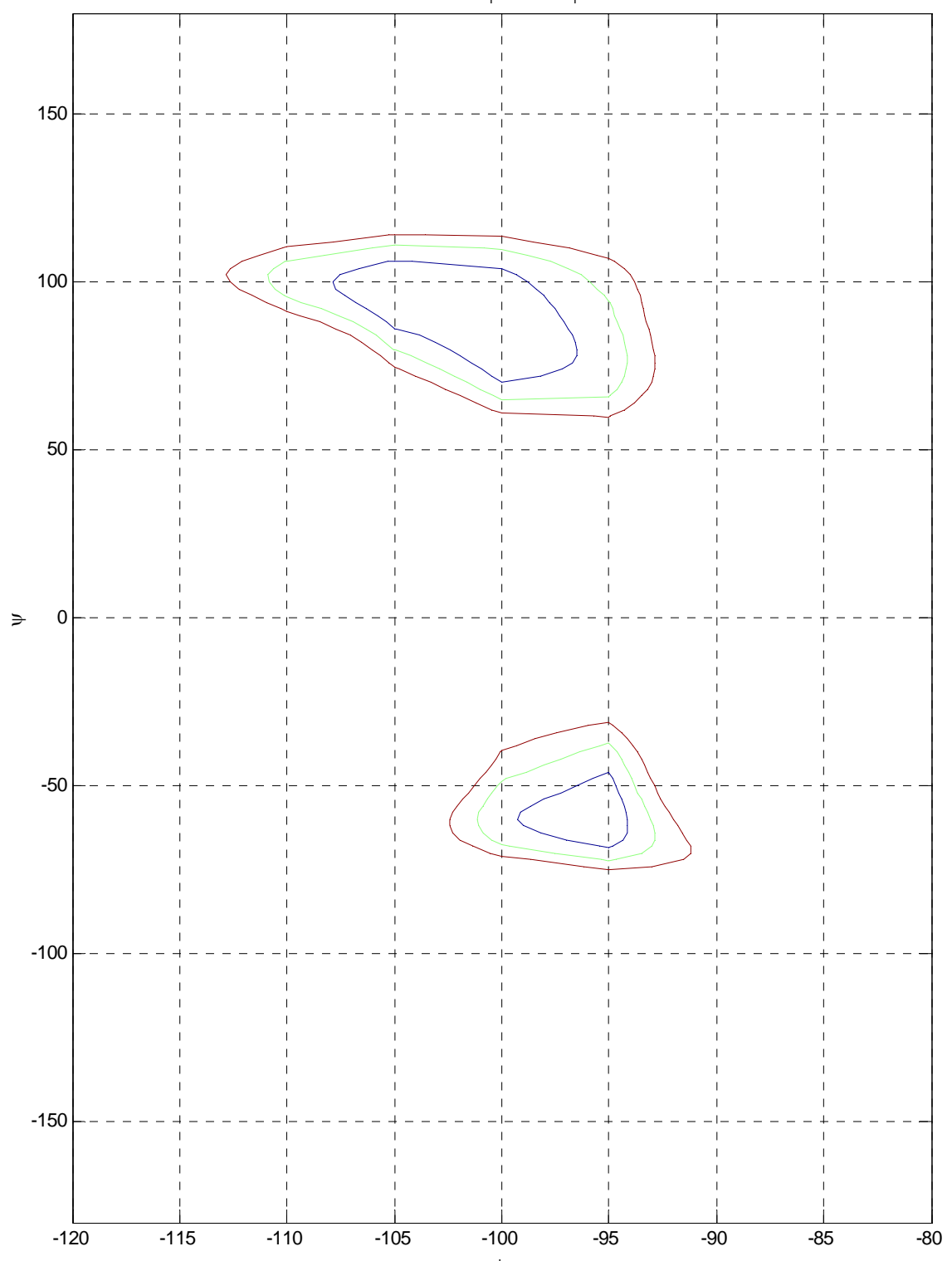

$t_{1}$ fit

Ramachandran $\chi^{2}$ map for FAG with step of 5 degrees Minimum at: $\phi=-105, \psi=110$

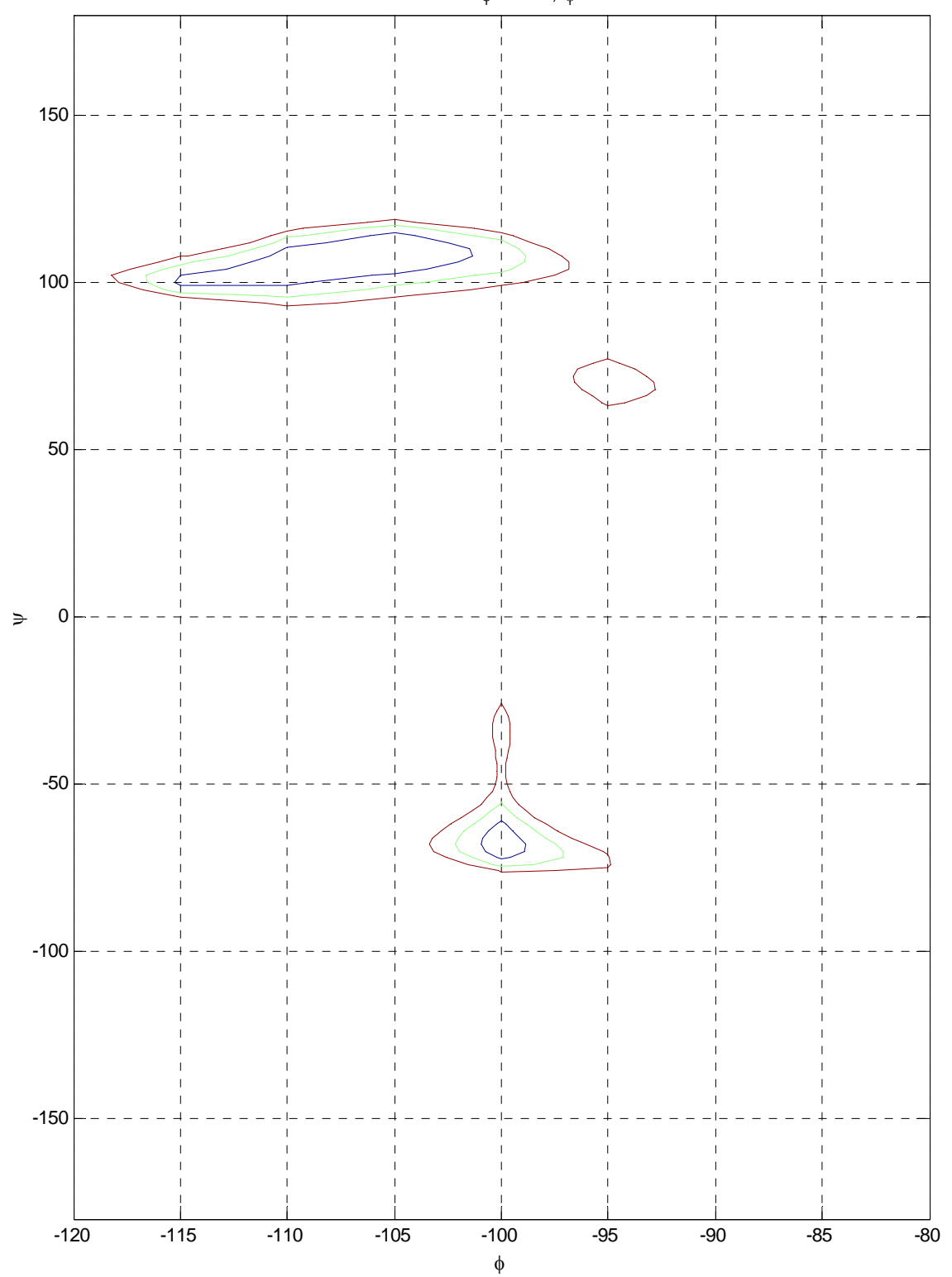




\section{0 degree AFG grid}

\section{Fourier Series}

Ramachandran $\chi^{2}$ map for AFG with step of 10 degrees Minimum at: $\phi=-100, \psi=90$

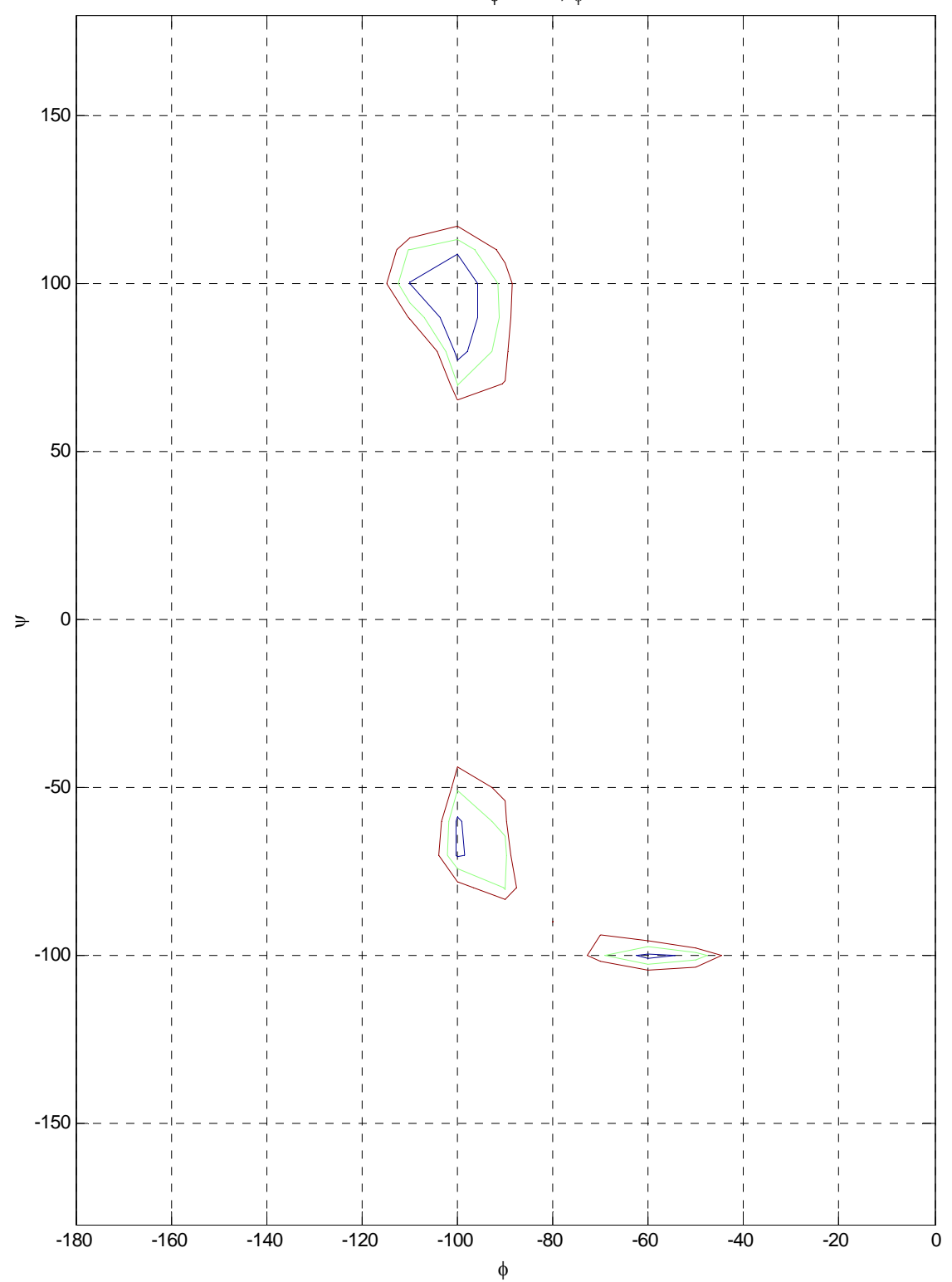

$t_{1}$ fit

Ramachandran $\chi^{2}$ map for AFG with step of 10 degrees Minimum at: $\phi=-100, \psi=-70$

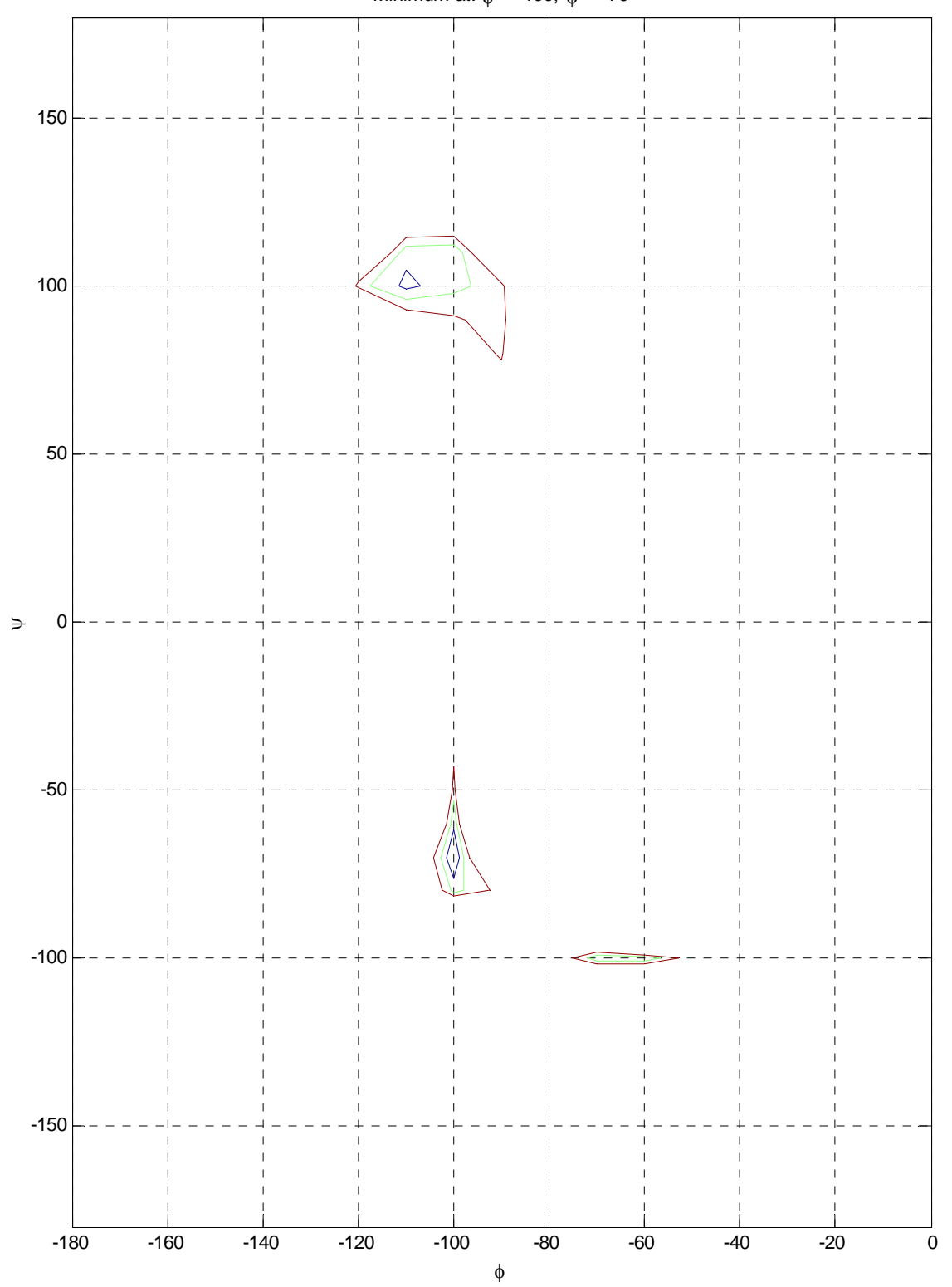




\section{$5 \times 2$ degree AFG grid}

\section{Fourier Series}

Ramachandran $\chi^{2}$ map for AFG with step of 5 degrees Minimum at: $\phi=-103, \psi=98$

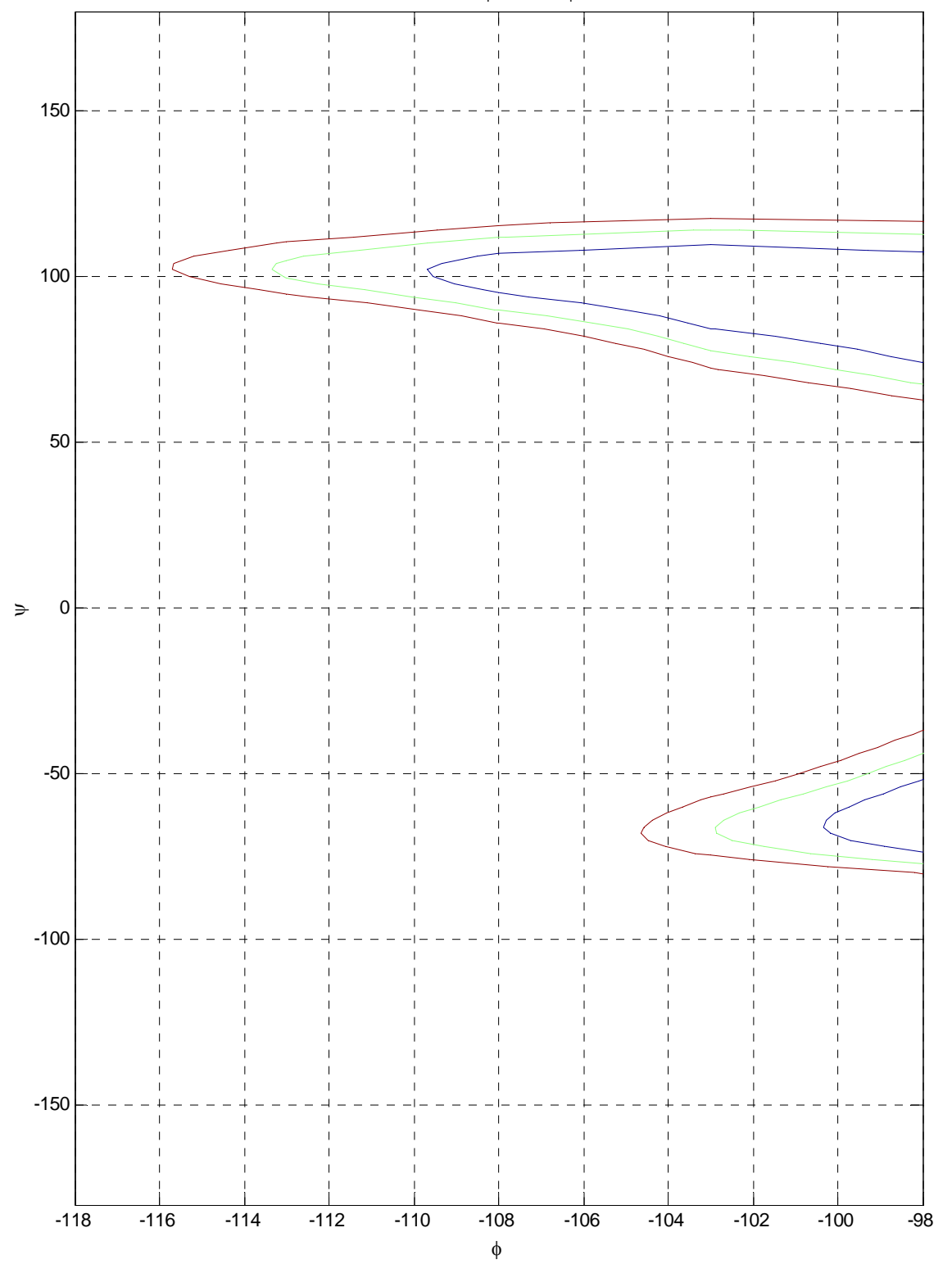

$t_{1}$ fit

Ramachandran $\chi^{2}$ map for AFG with step of 5 degrees Minimum at: $\phi=-98, \psi=-72$

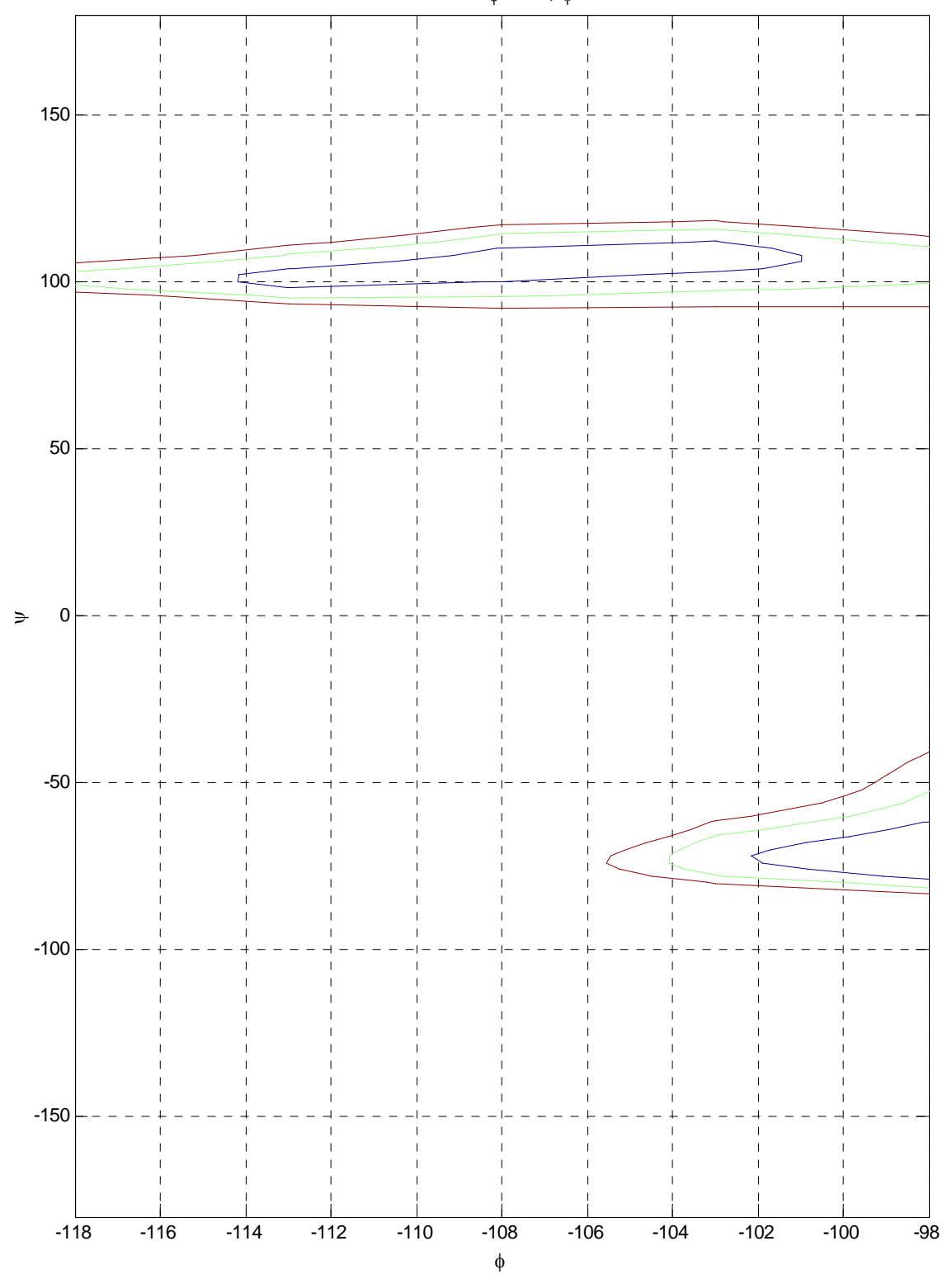




\section{5x2 degree GEN grid}

\section{Fourier Series}

Ramachandran $\chi^{2}$ map for AFG with step of 5 degrees Minimum at: $\phi=-95, \psi=-62$

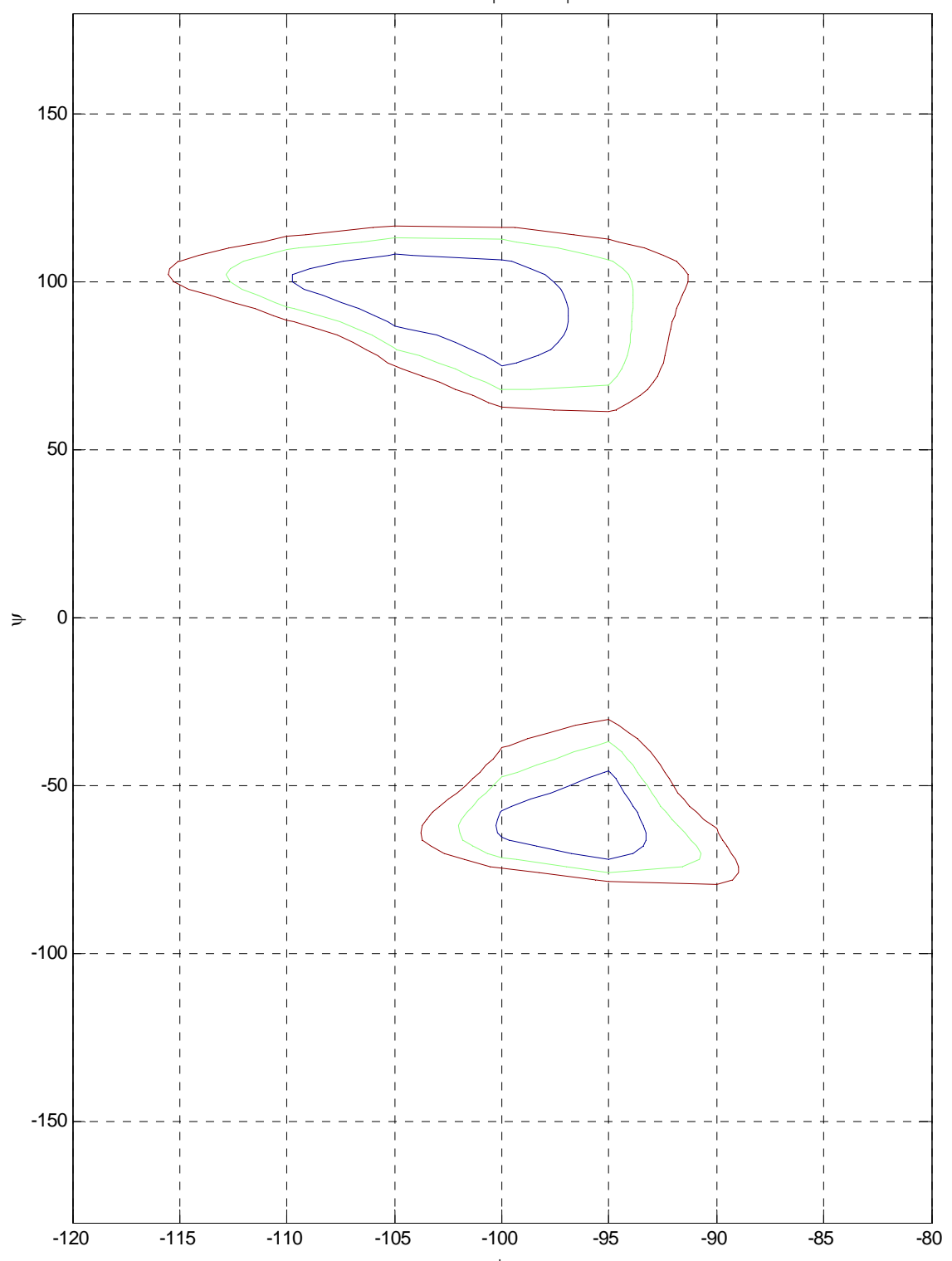

$t_{1}$ fit

Ramachandran $\chi^{2}$ map for AFG with step of 5 degrees Minimum at: $\phi=-100, \psi=-68$

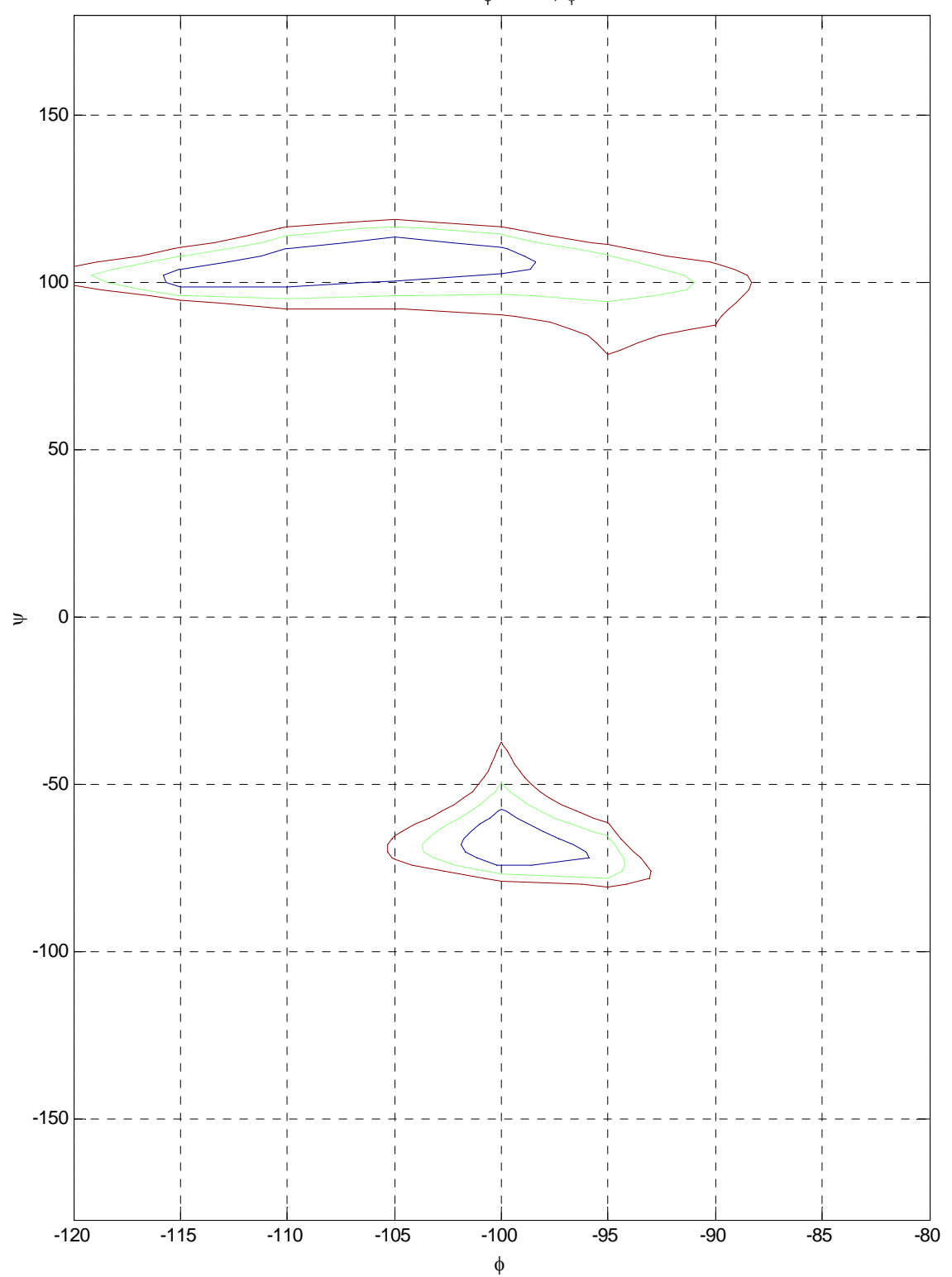




\section{0 degree GFF grid}

\section{Fourier Series}

Ramachandran $\chi^{2}$ map for GFF with step of 10 degrees Minimum at: $\phi=-130, \psi=-60$

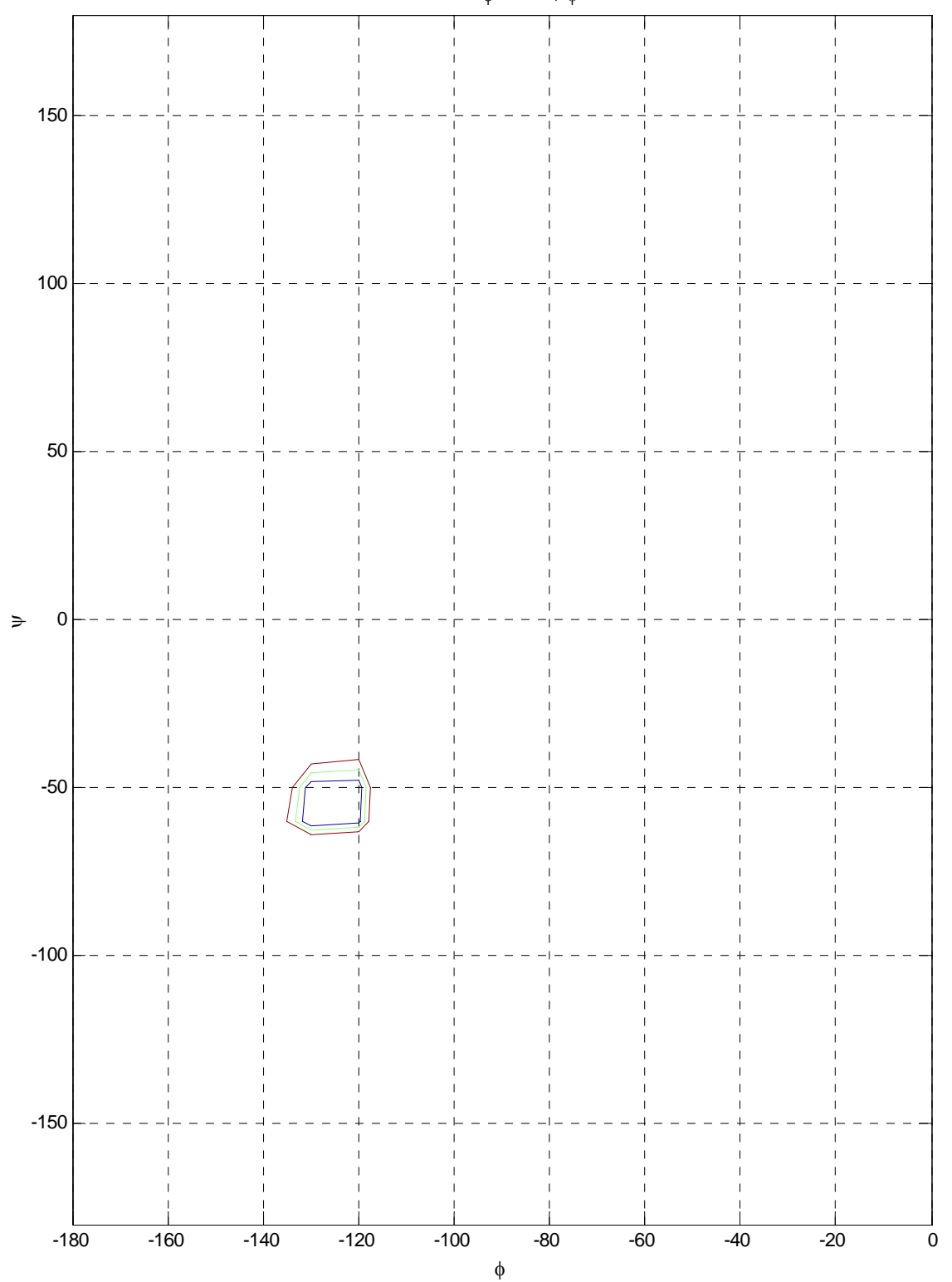

$t_{1}$ fit

Ramachandran $\chi^{2}$ map for GFF with step of 10 degrees Minimum at: $\phi=-130, \psi=-60$

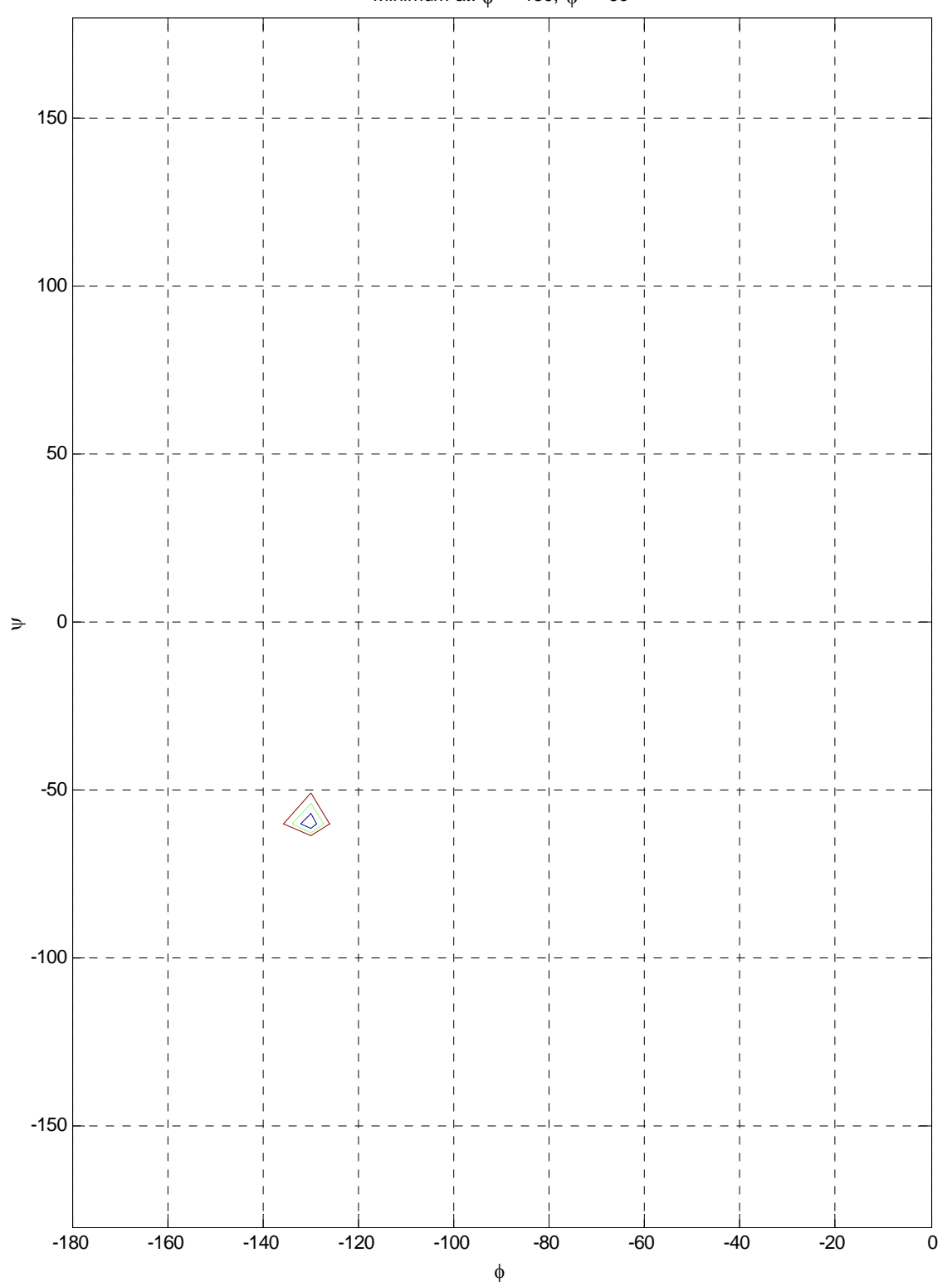




\section{5x2 degree GFF grid}

\section{Fourier Series}

Ramachandran $\chi^{2}$ map for GFF with step of 5 degrees Minimum at: $\phi=-127, \psi=-56$

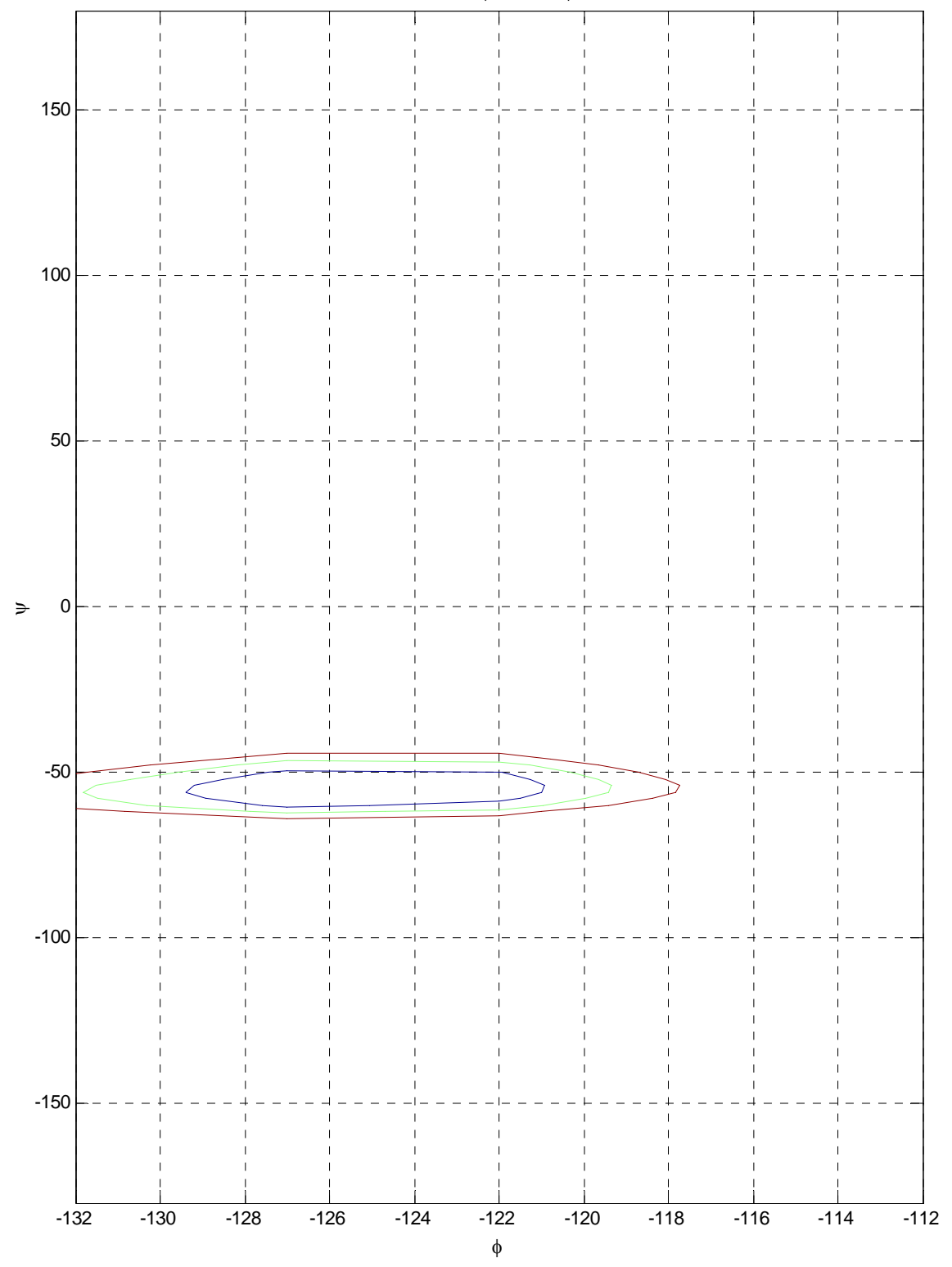

$t_{1}$ fit

Ramachandran $\chi^{2}$ map for GFF with step of 5 degrees Minimum at: $\phi=-132, \psi=-60$

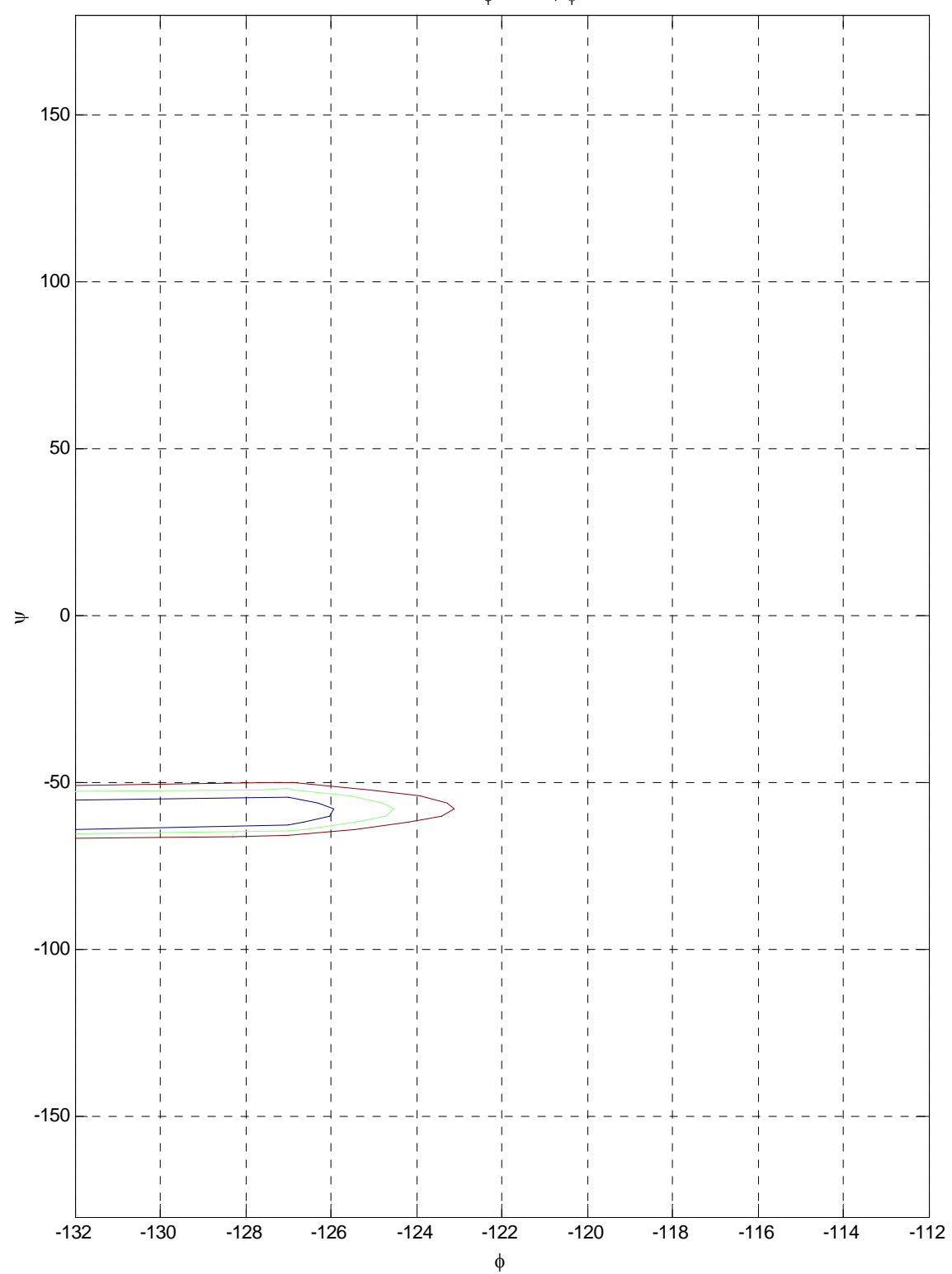




\section{5×2 degree GEN grid}

\section{Fourier Series}

Ramachandran $\chi^{2}$ map for GFF with step of 5 degrees Minimum at: $\phi=-135, \psi=32$

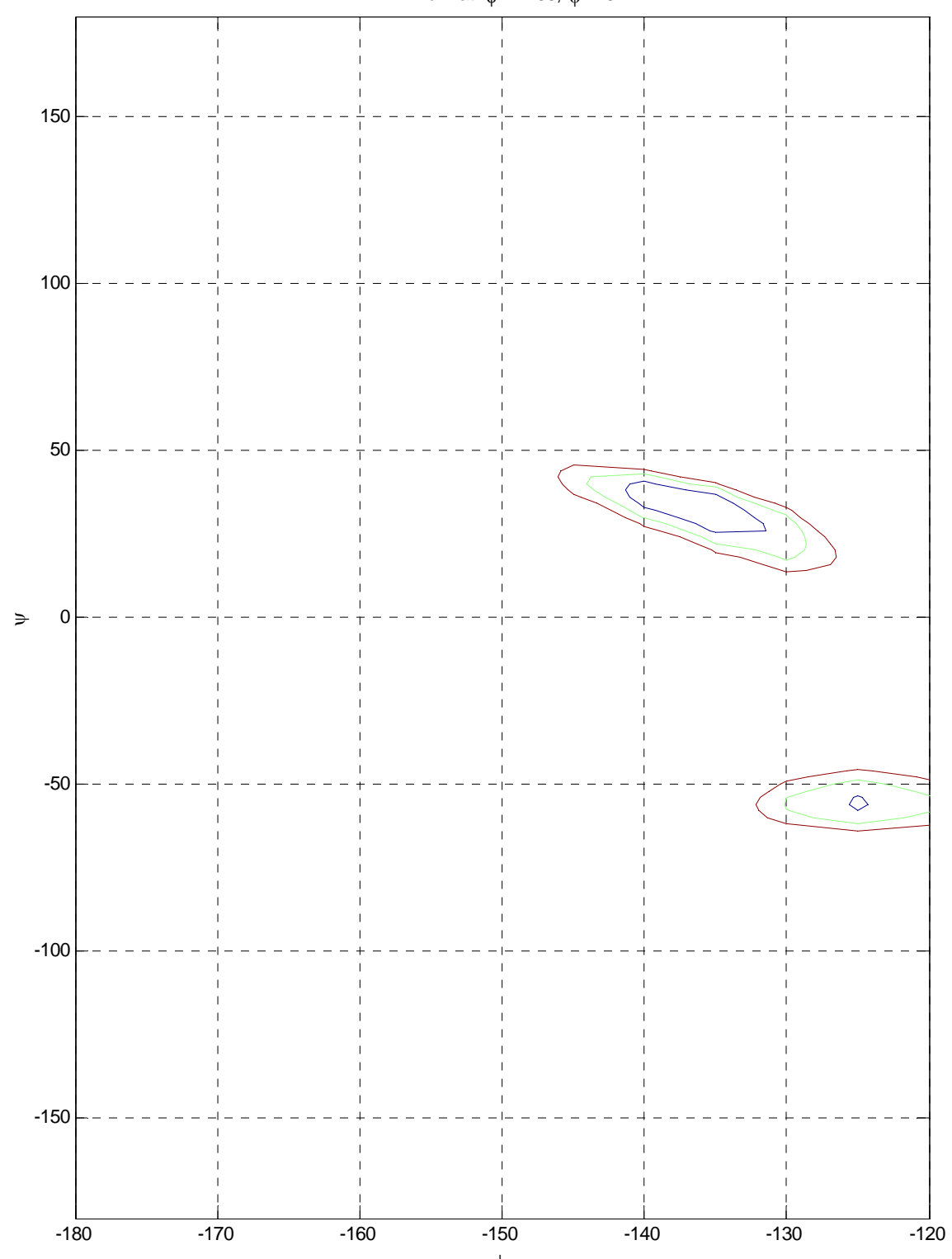

$t_{1}$ fit

Ramachandran $\chi^{2}$ map for GFF with step of 5 degrees Minimum at: $\phi=-145, \psi=44$

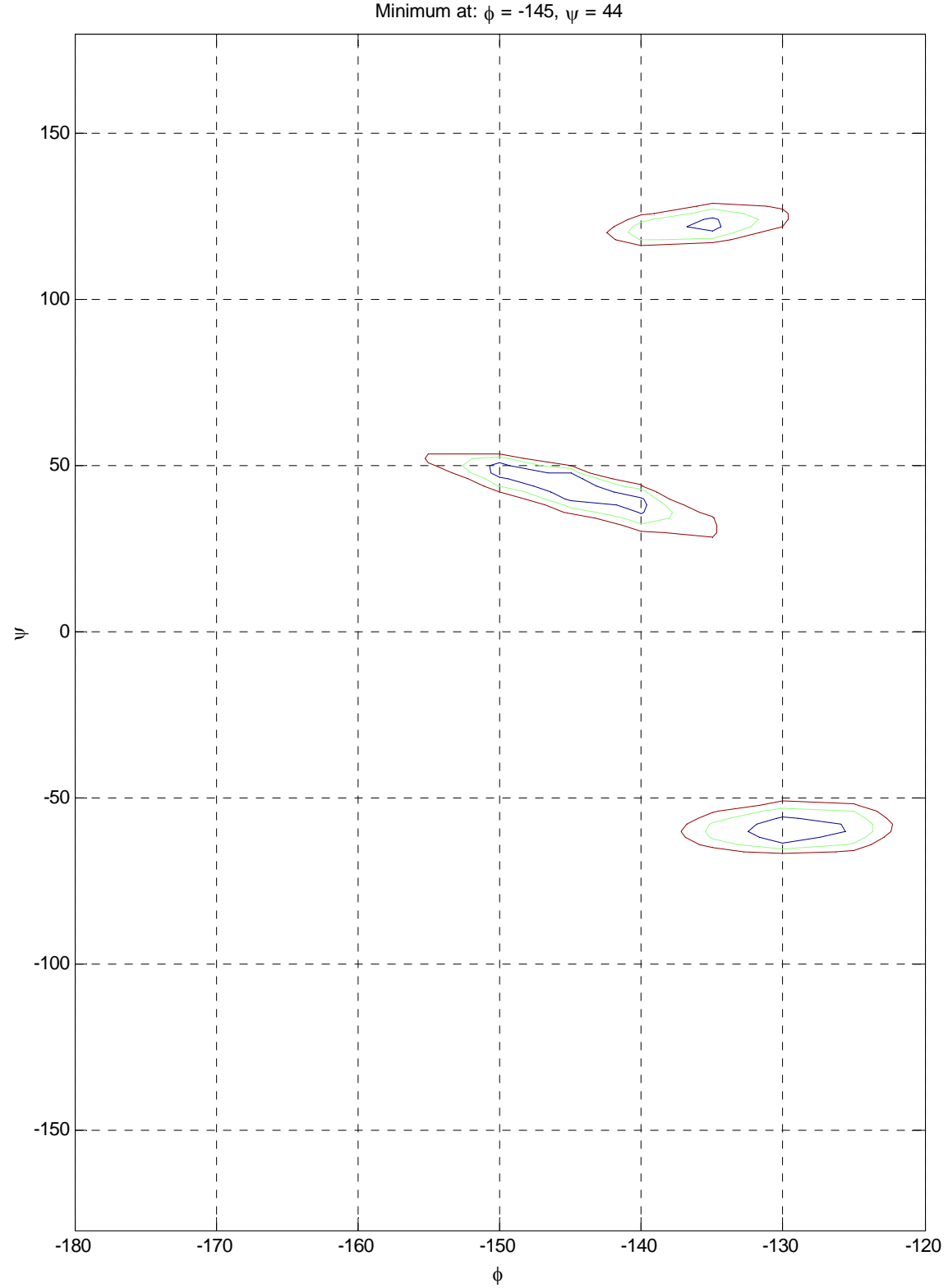




\section{$5 \times 2$ degree GFF grid}

\section{$-127-56 \quad \chi^{2}=205$}
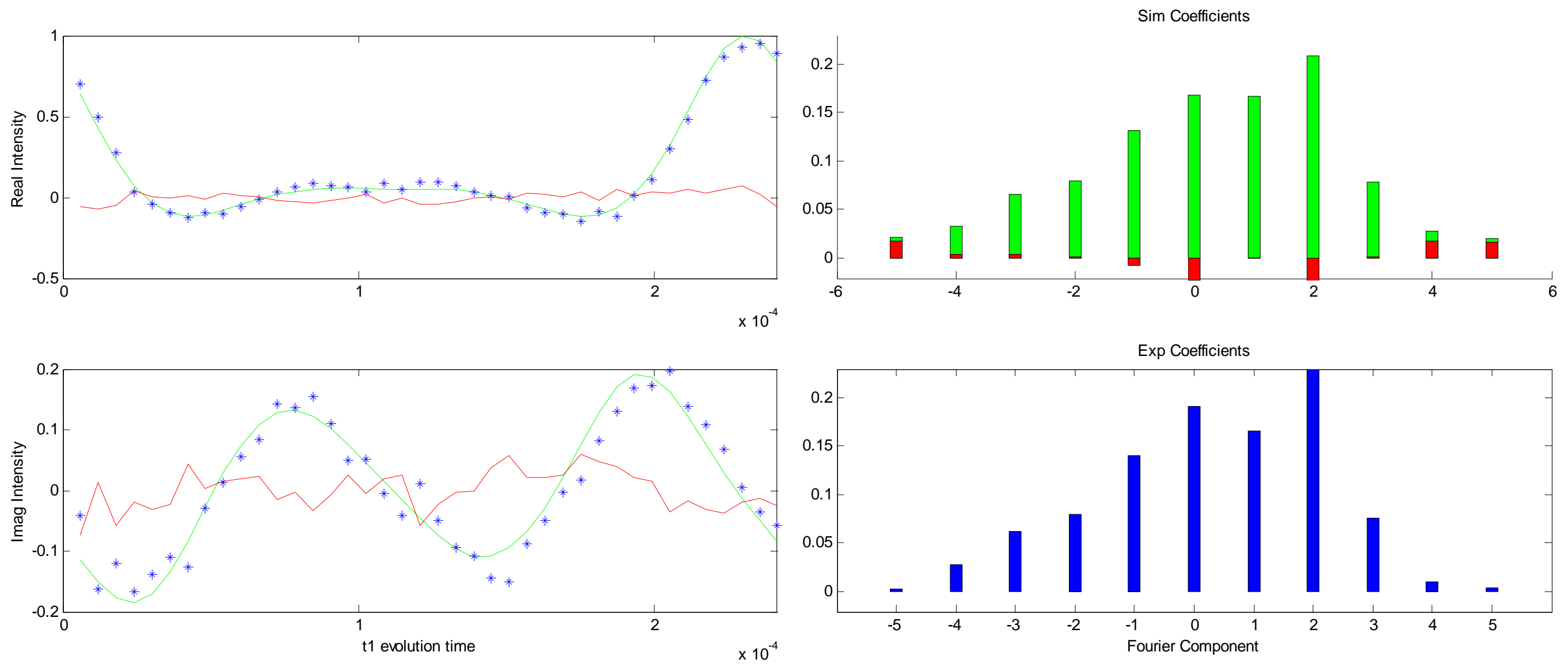


\section{0 degree GFG grid}

\section{Fourier Series}

Ramachandran $\chi^{2}$ map for GFG with step of 10 degrees Minimum at: $\phi=-60, \psi=-120$

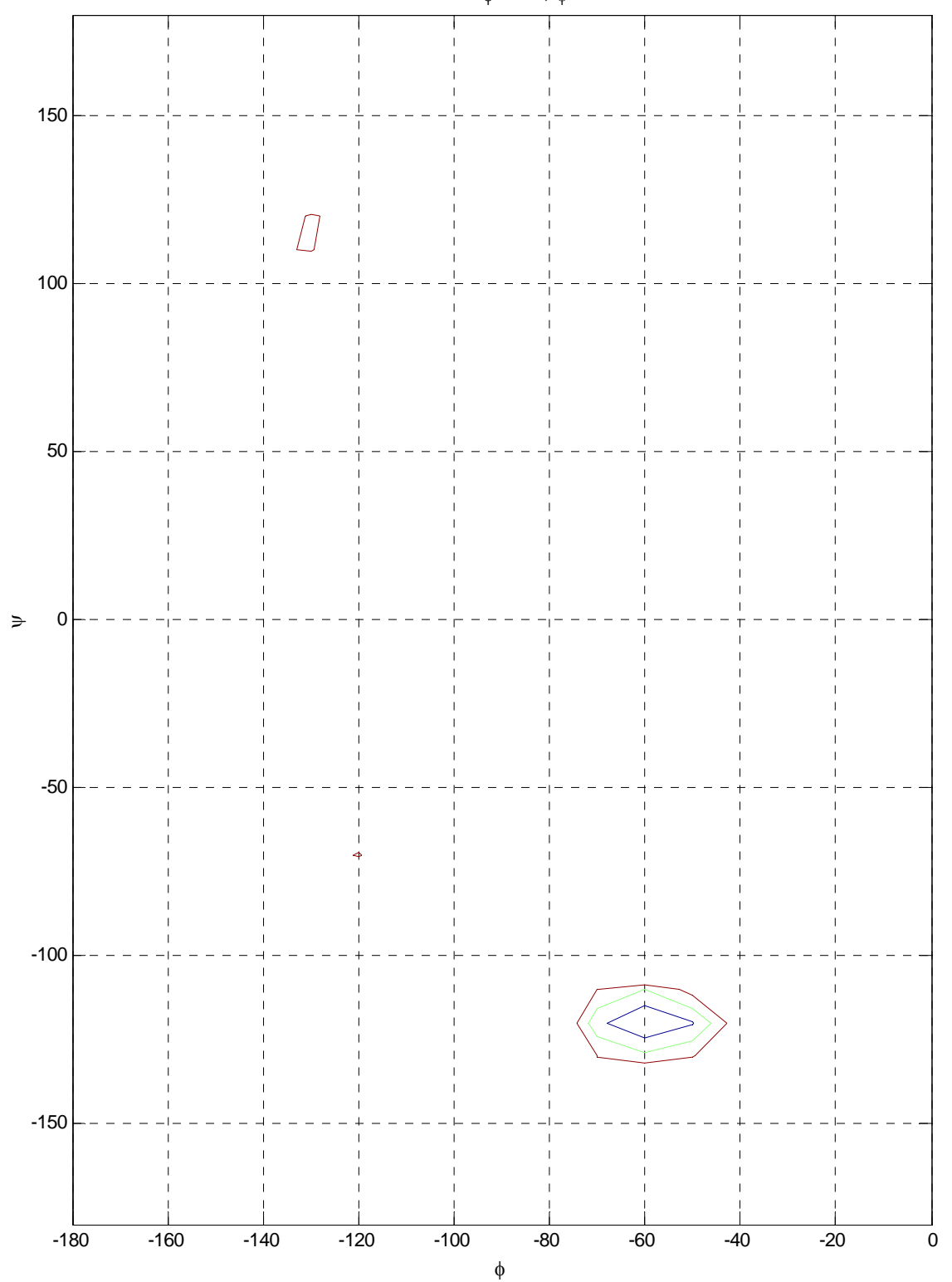

$t_{1}$ fit

Ramachandran $\chi^{2}$ map for GFG with step of 10 degrees Minimum at: $\phi=-130, \psi=-70$

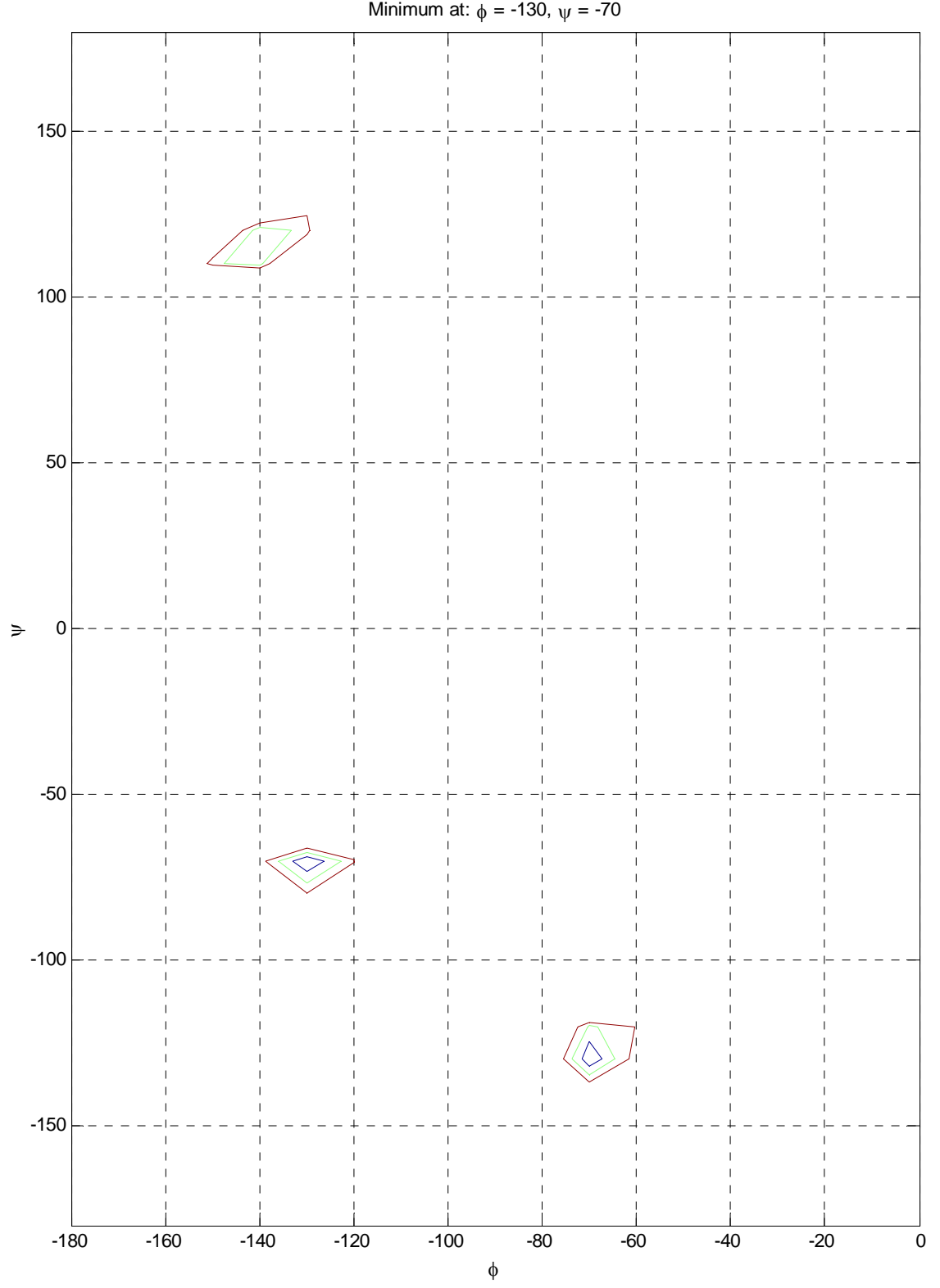




\section{5×2 degree GFG grid}

\section{Fourier Series}

Ramachandran $\chi^{2}$ map for GFG with step of 5 degrees Minimum at: $\phi=-128, \psi=116$

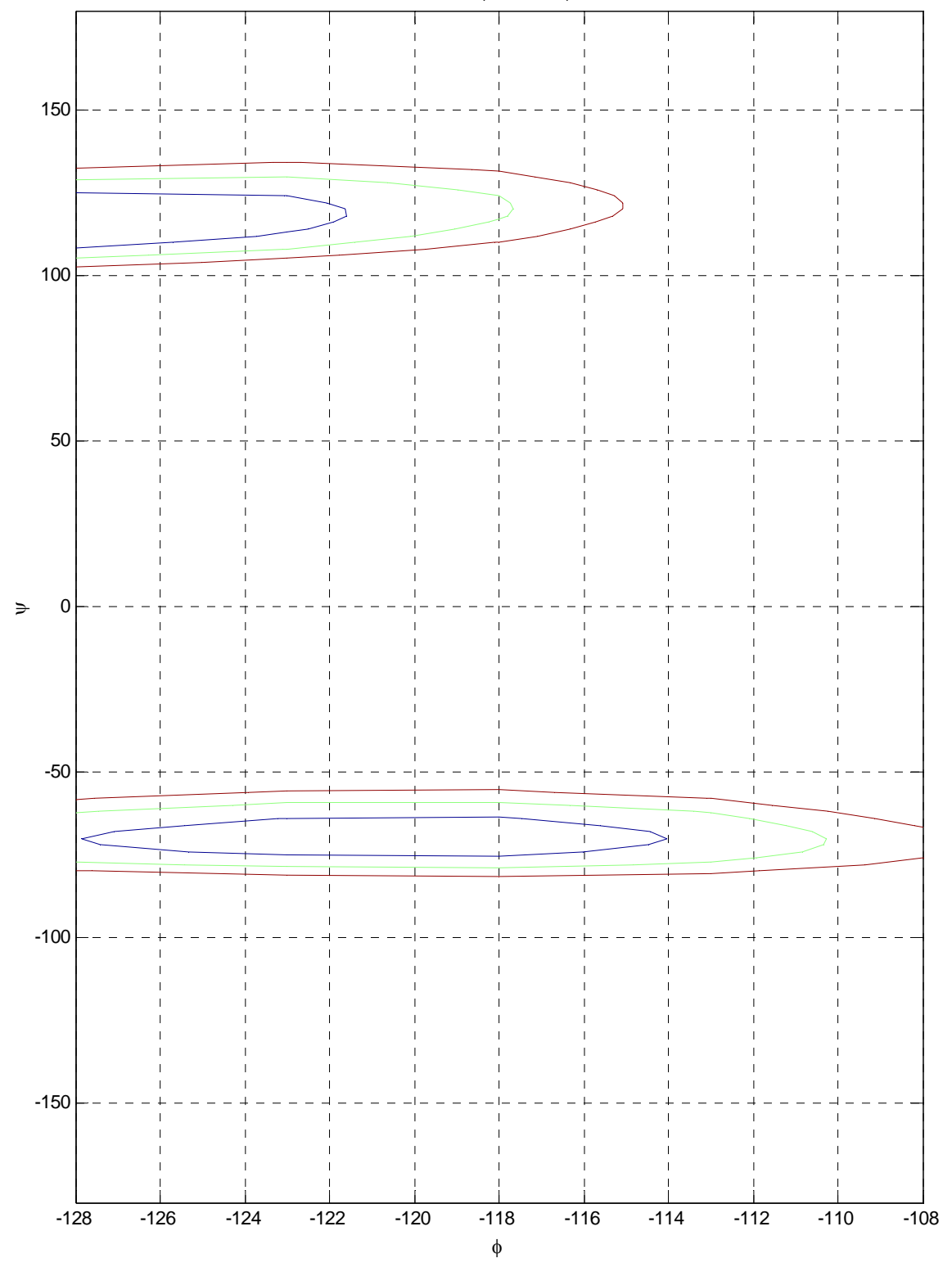

$t_{1}$ fit

Ramachandran $\chi^{2}$ map for GFG with step of 5 degrees Minimum at: $\phi=-128, \psi=-74$

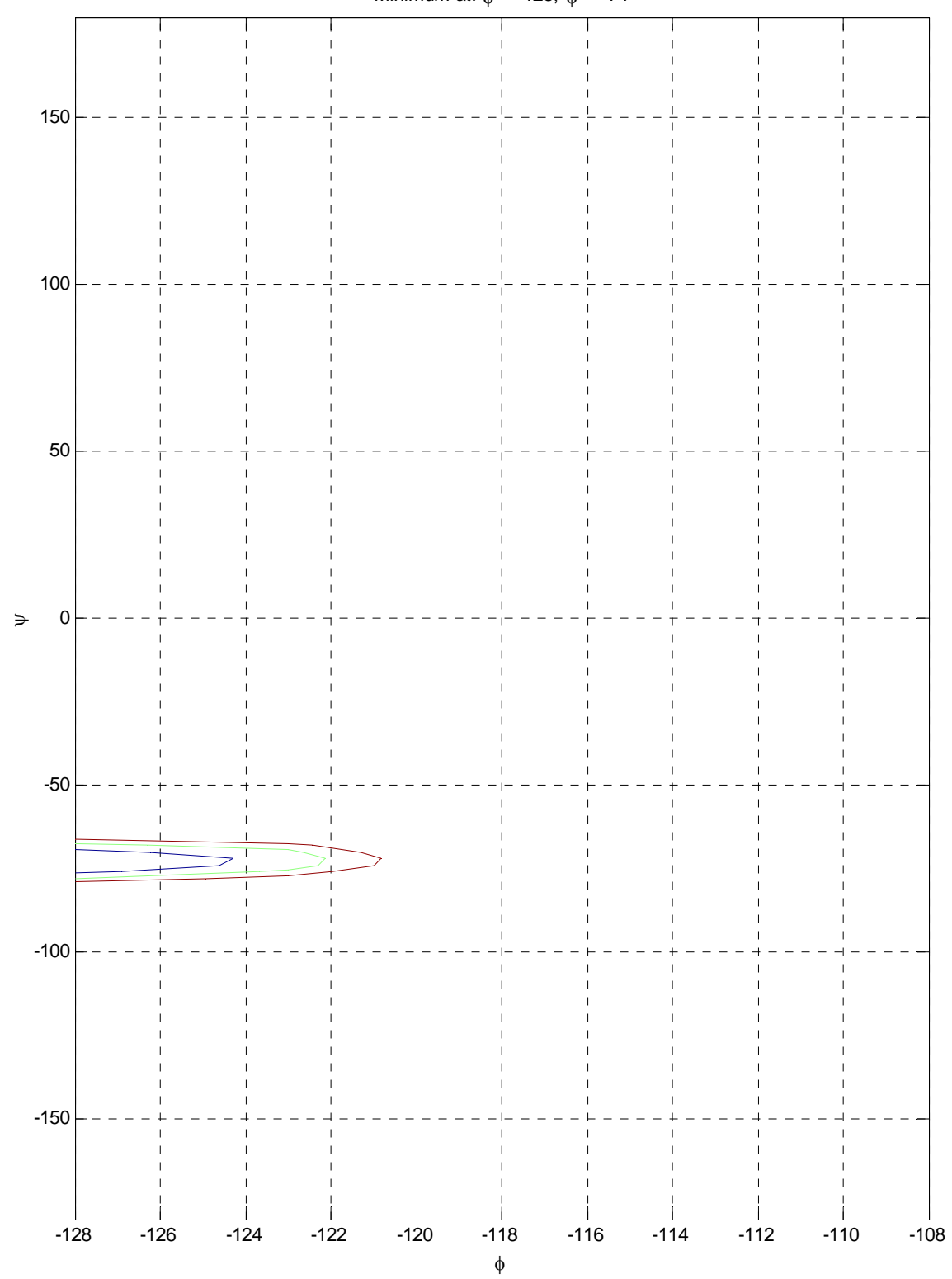




\section{5×2 degree GEN grid}

\section{Fourier Series}

Ramachandran $\chi^{2}$ map for GFG with step of 5 degrees Minimum at: $\phi=-130, \psi=116$

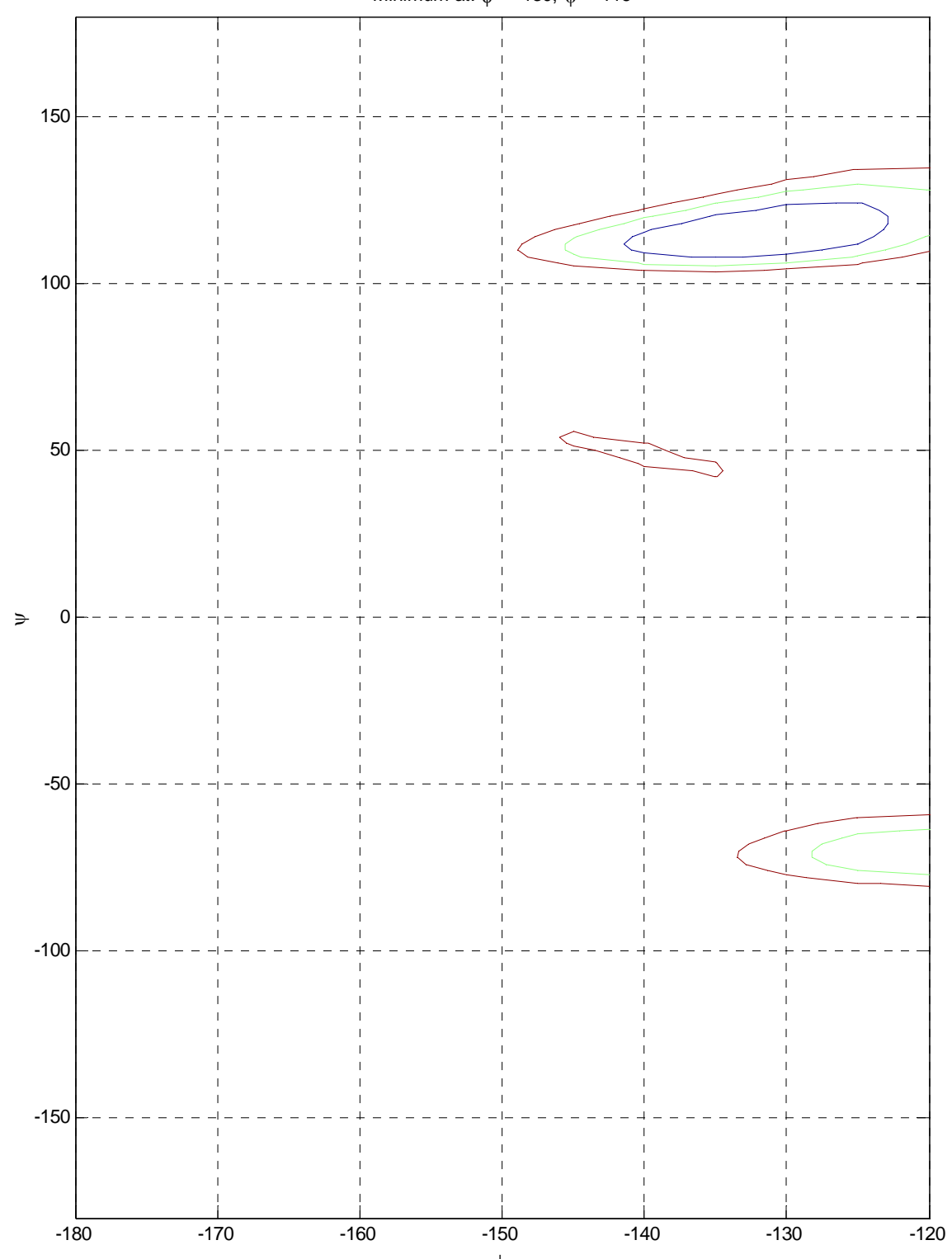

$t_{1}$ fit

Ramachandran $\chi^{2}$ map for GFG with step of 5 degrees Minimum at: $\phi=-130, \psi=-74$

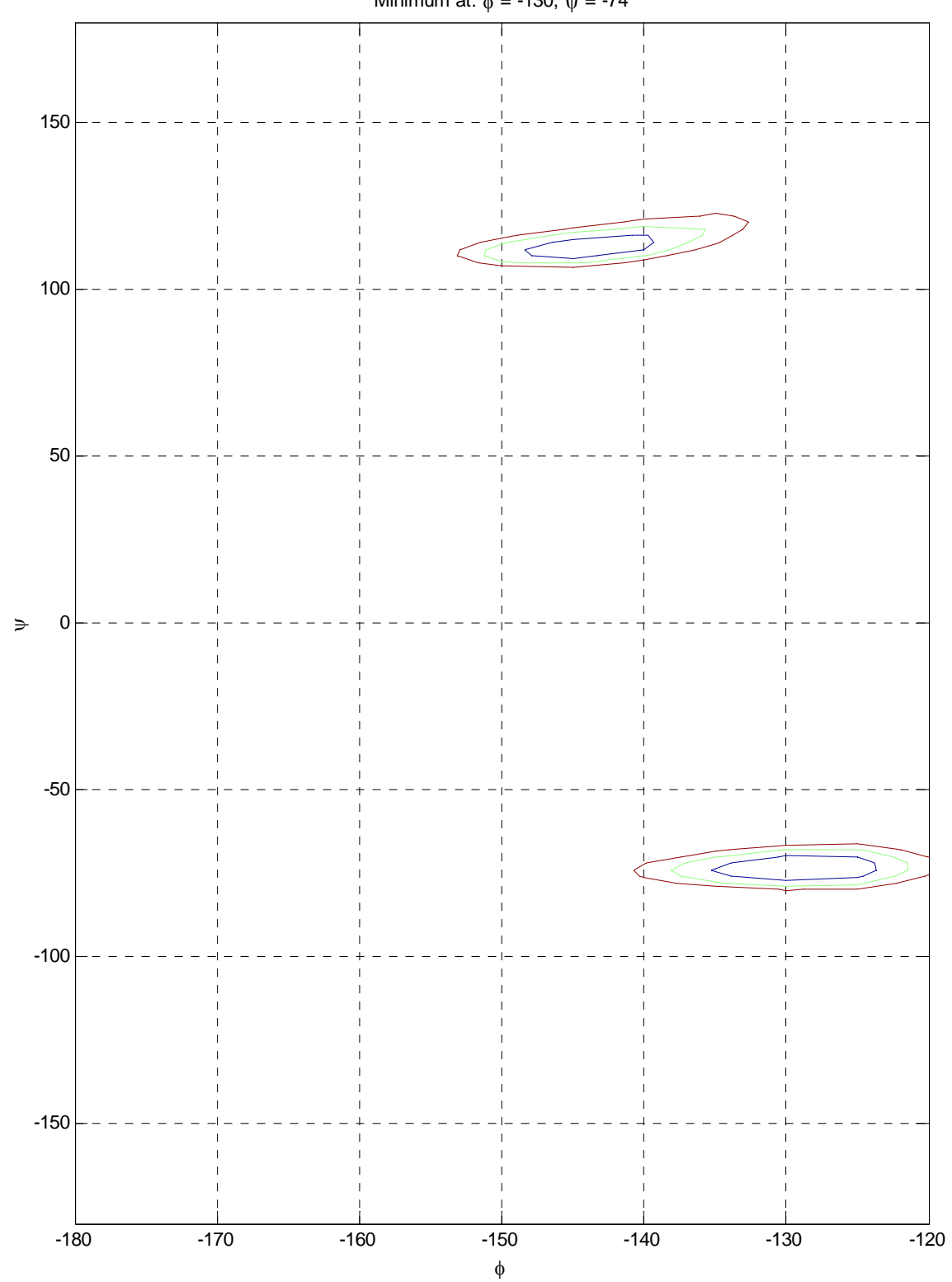




\section{0 degree VGG grid}

\section{Fourier Series}

Ramachandran $\chi^{2}$ map for VGG with step of 10 degrees Minimum at: $\phi=-30, \psi=-170$

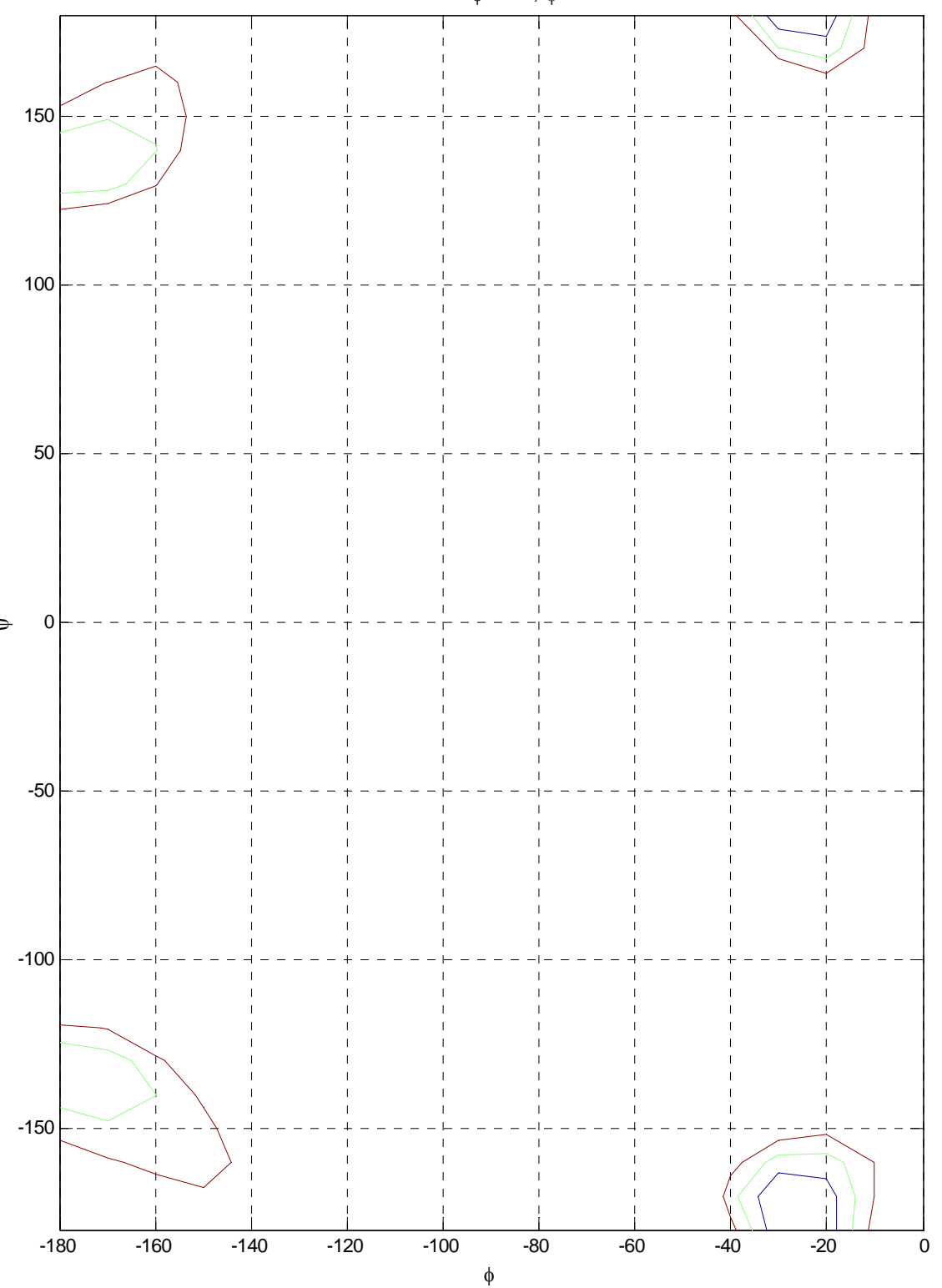

$t_{1}$ fit

Ramachandran $\chi^{2}$ map for VGG with step of 10 degrees Minimum at: $\phi=-180, \psi=130$

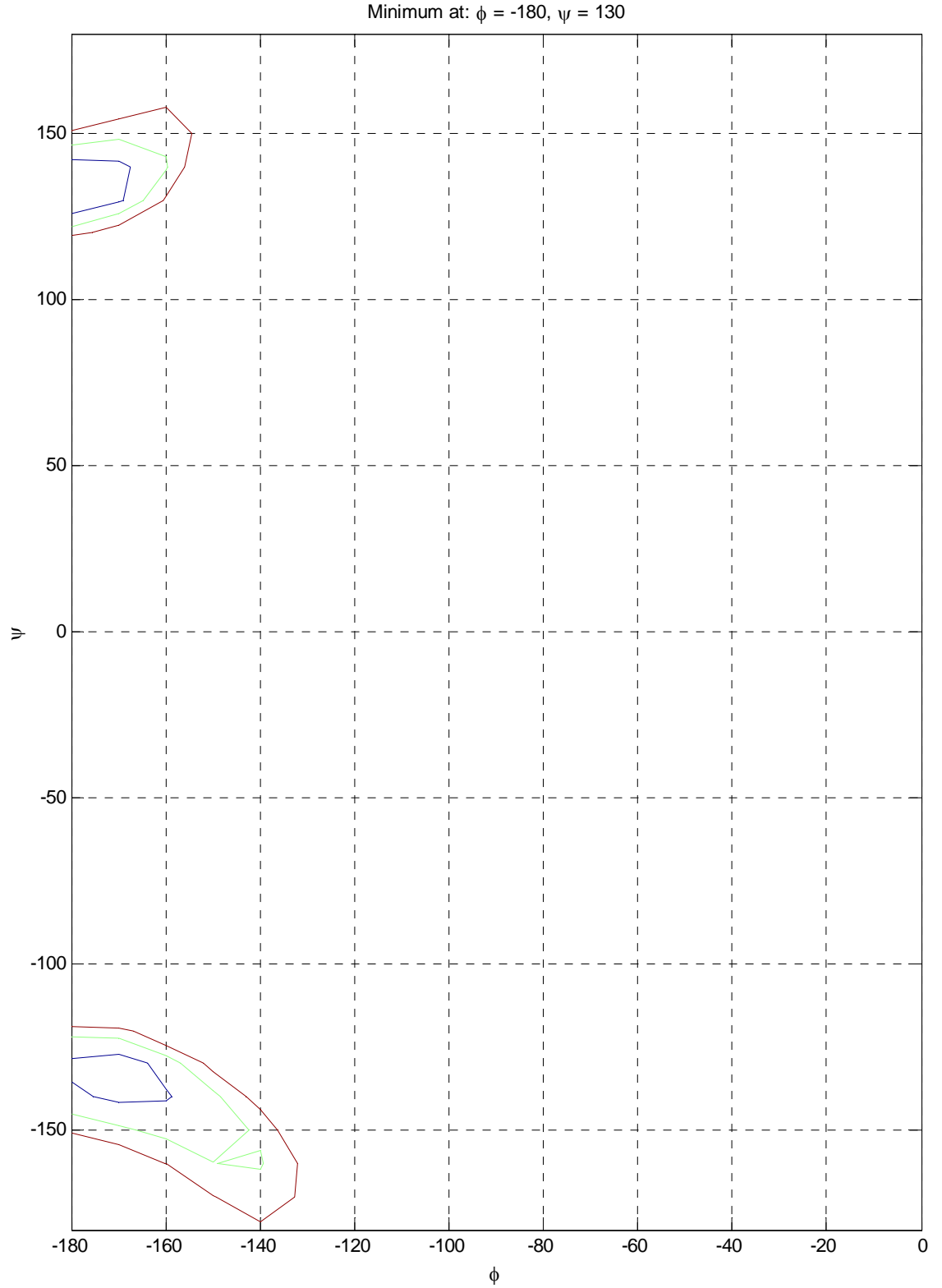




\section{5×2 degree VGG grid}

\section{Fourier Series}

Ramachandran $\chi^{2}$ map for VGG with step of 5 degrees Minimum at: $\phi=-168, \psi=138$

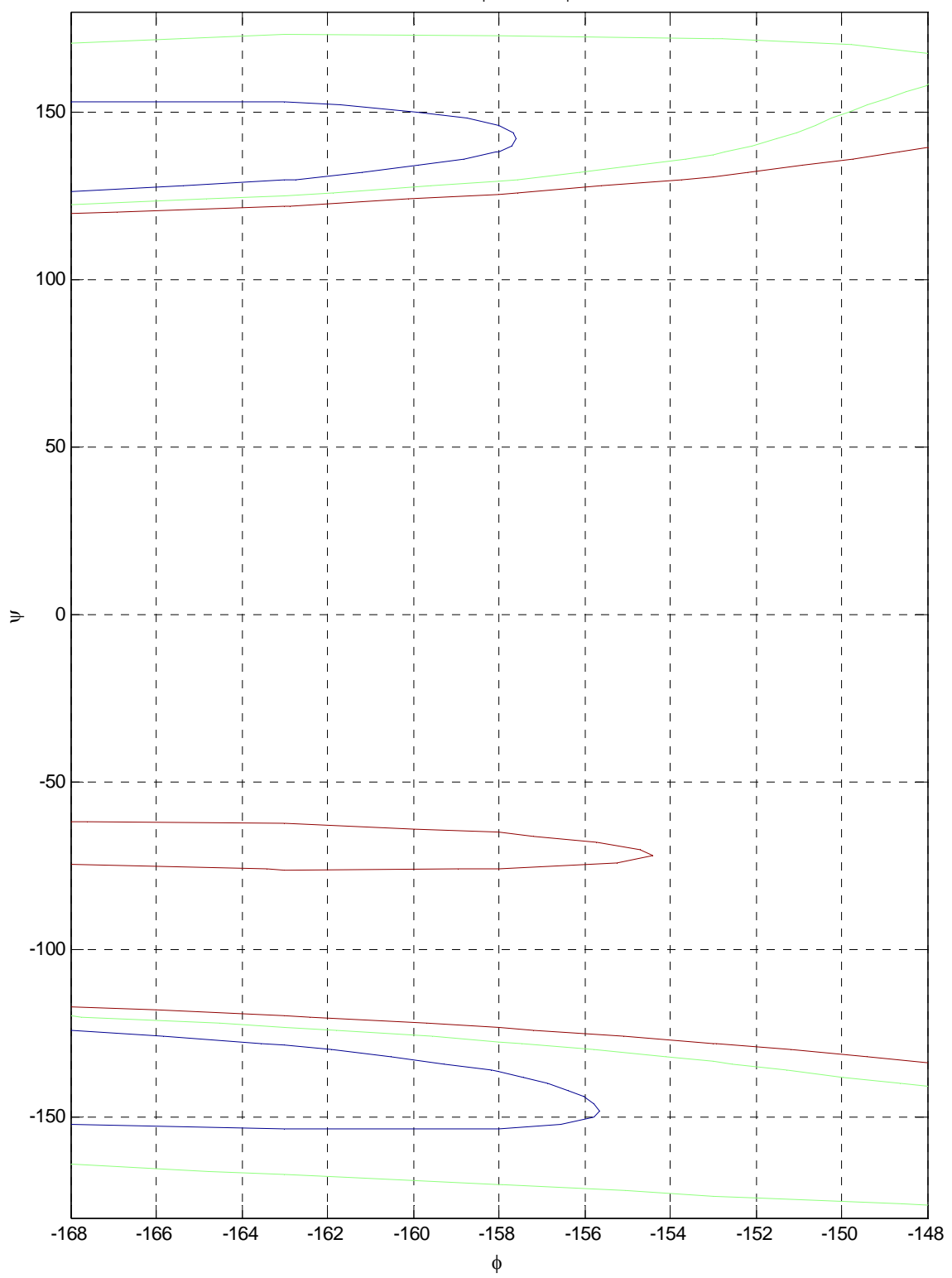

$t_{1}$ fit

Ramachandran $\chi^{2}$ map for VGG with step of 5 degrees Minimum at: $\phi=-168, \psi=-134$

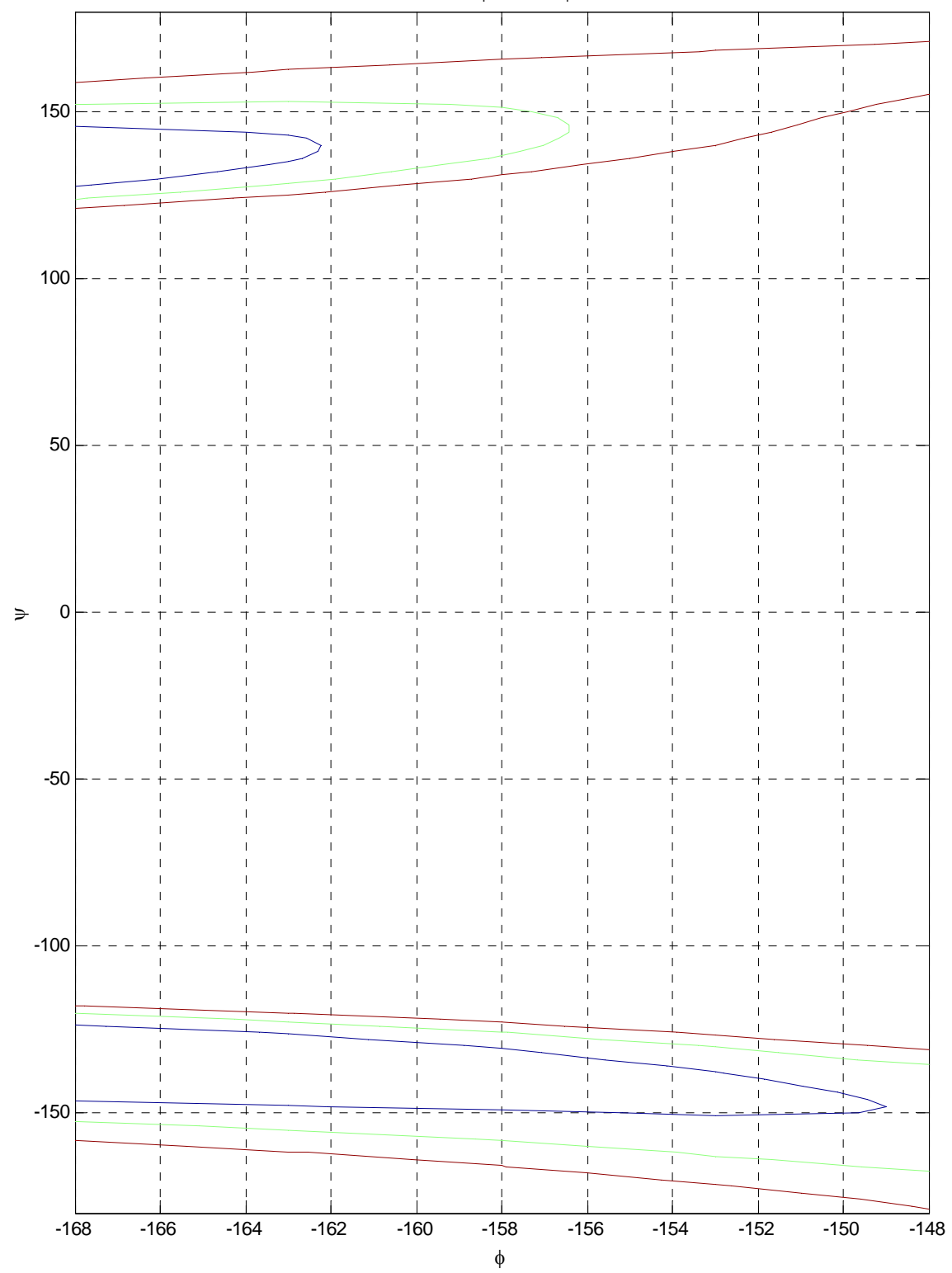




\section{5×2 degree GEN grid}

\section{Fourier Series}

Ramachandran $\chi^{2}$ map for VGG with step of 5 degrees Minimum at: $\phi=-175, \psi=-132$

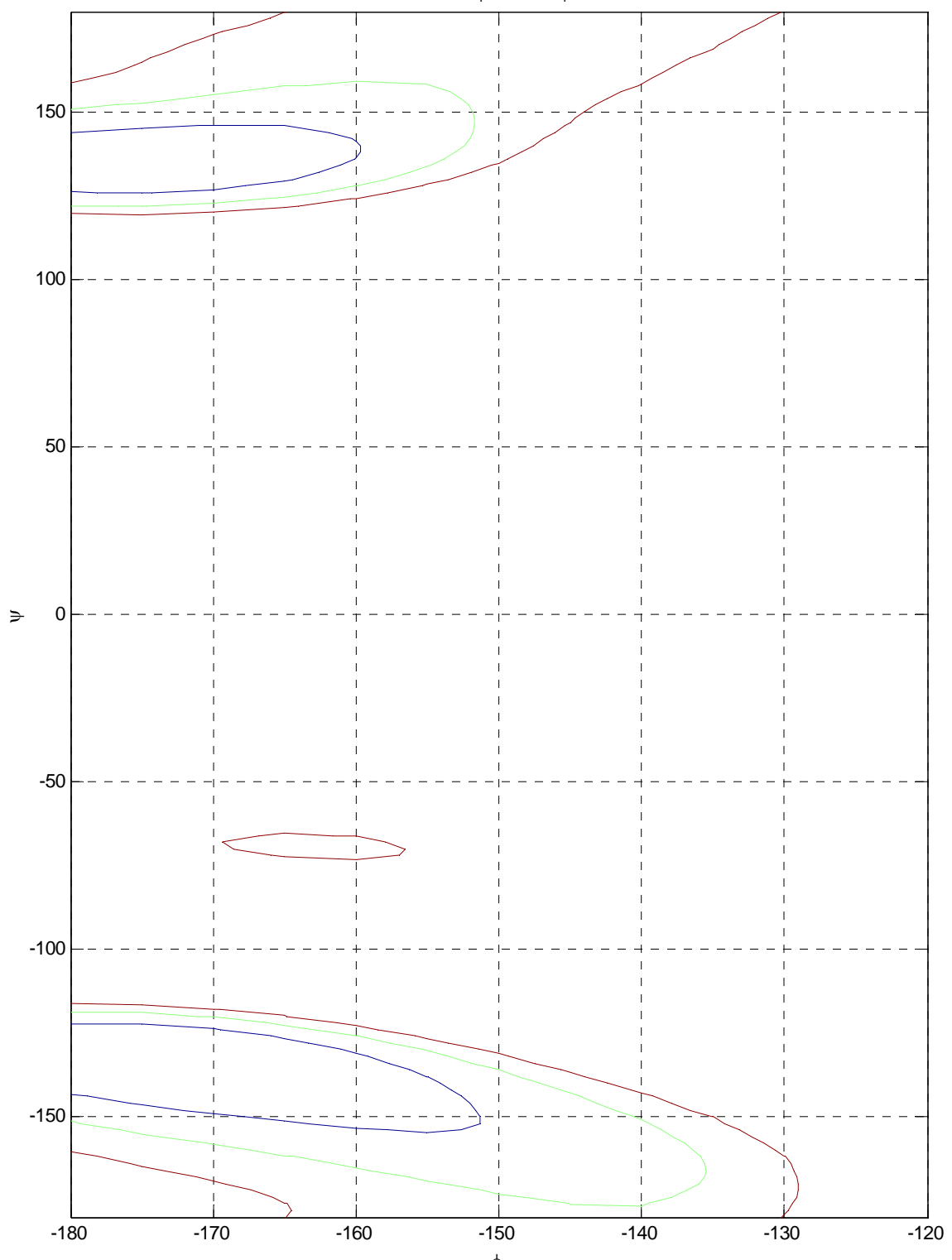

$t_{1}$ fit

Ramachandran $\chi^{2}$ map for VGG with step of 5 degrees Minimum at: $\phi=-180, \psi=132$

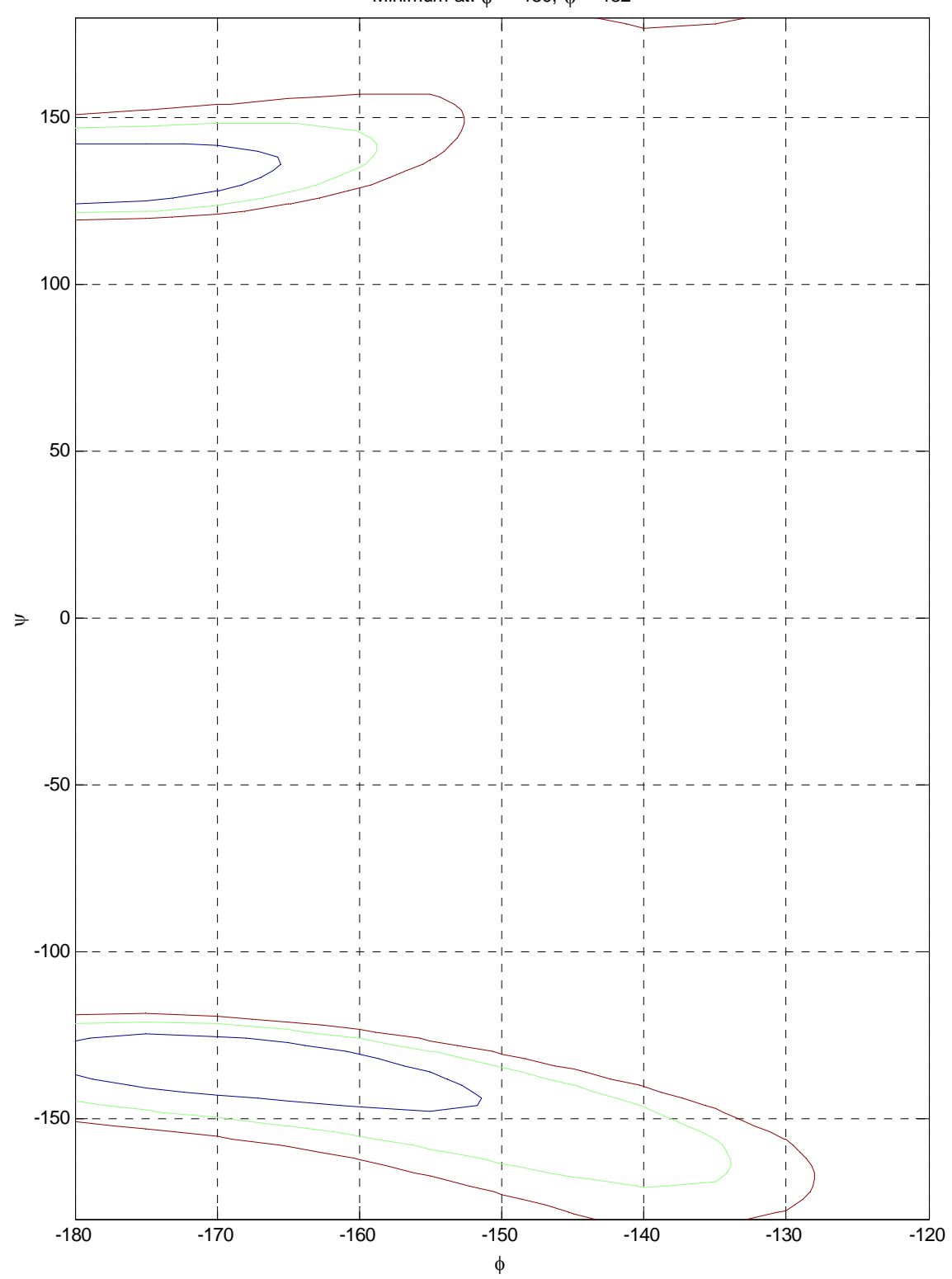




\section{0 degree AAA grid}

\section{Fourier Series}

Ramachandran $\chi^{2}$ map for AAA with step of 10 degrees Minimum at: $\phi=-160, \psi=140$

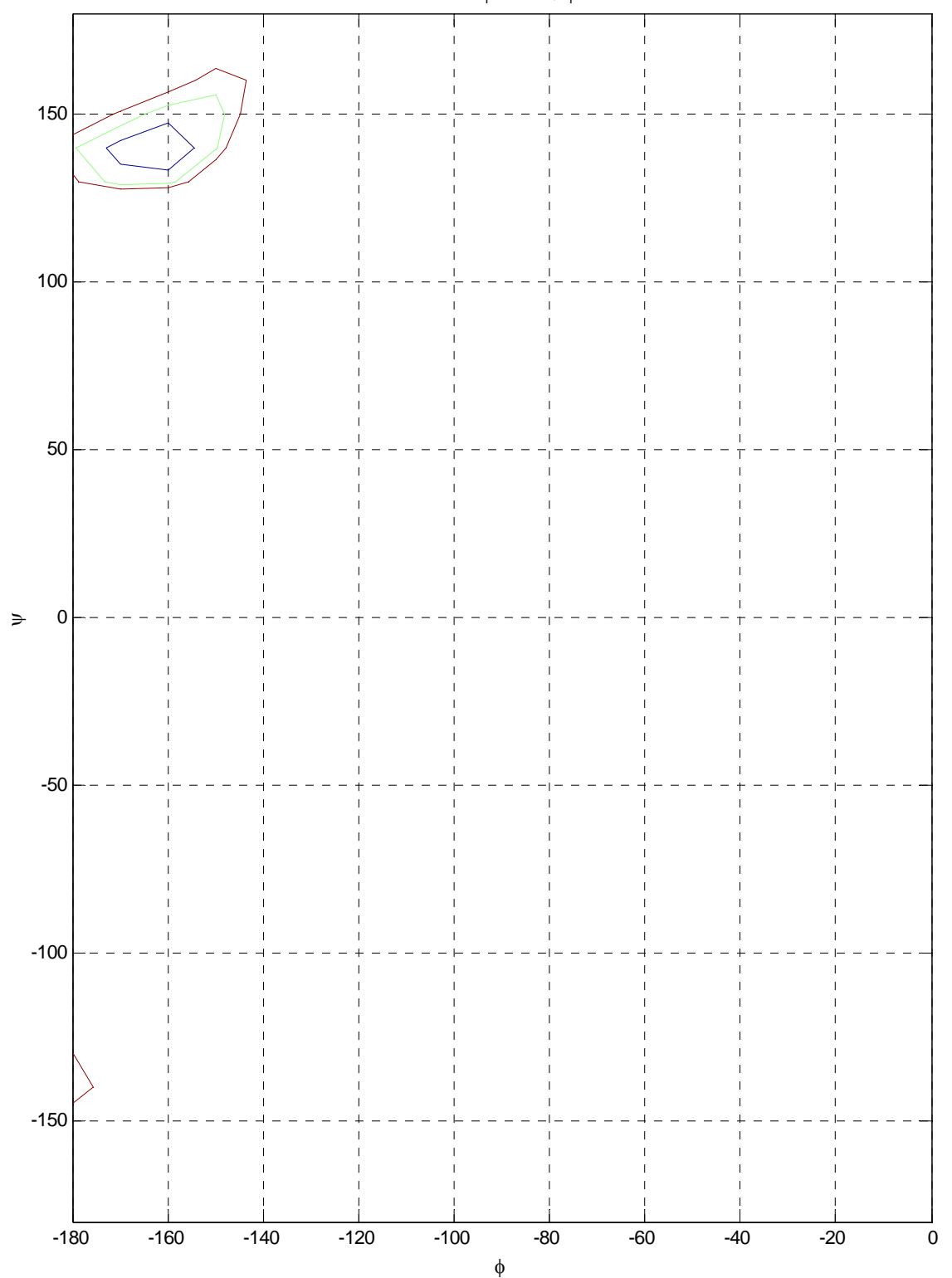

$t_{1}$ fit

Ramachandran $\chi^{2}$ map for AAA with step of 10 degrees Minimum at: $\phi=-160, \psi=140$

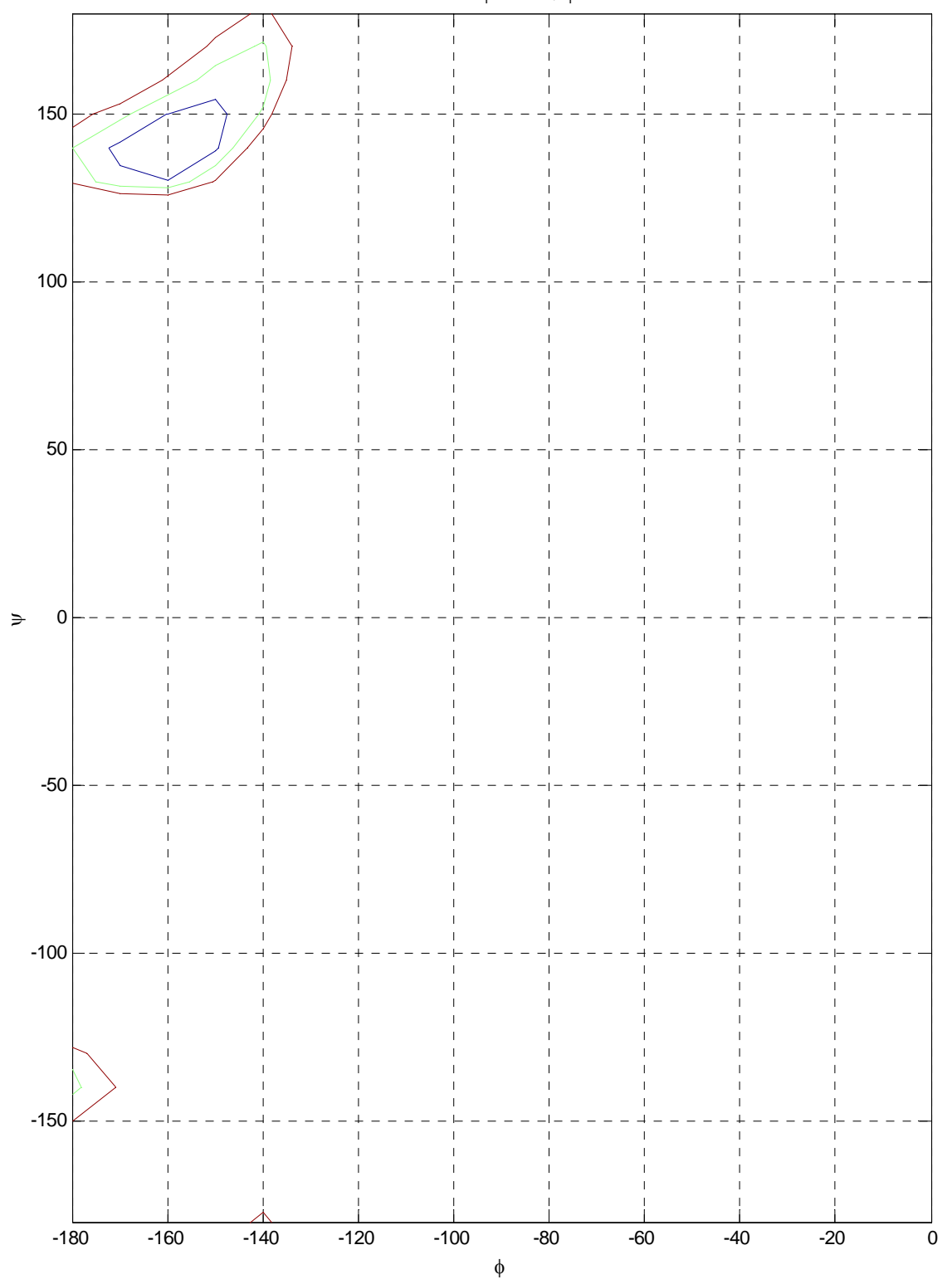




\section{$5 \times 2$ degree AAA grid}

\section{Fourier Series}

Ramachandran $\chi^{2}$ map for AAA with step of 5 degrees Minimum at: $\phi=-157, \psi=140$

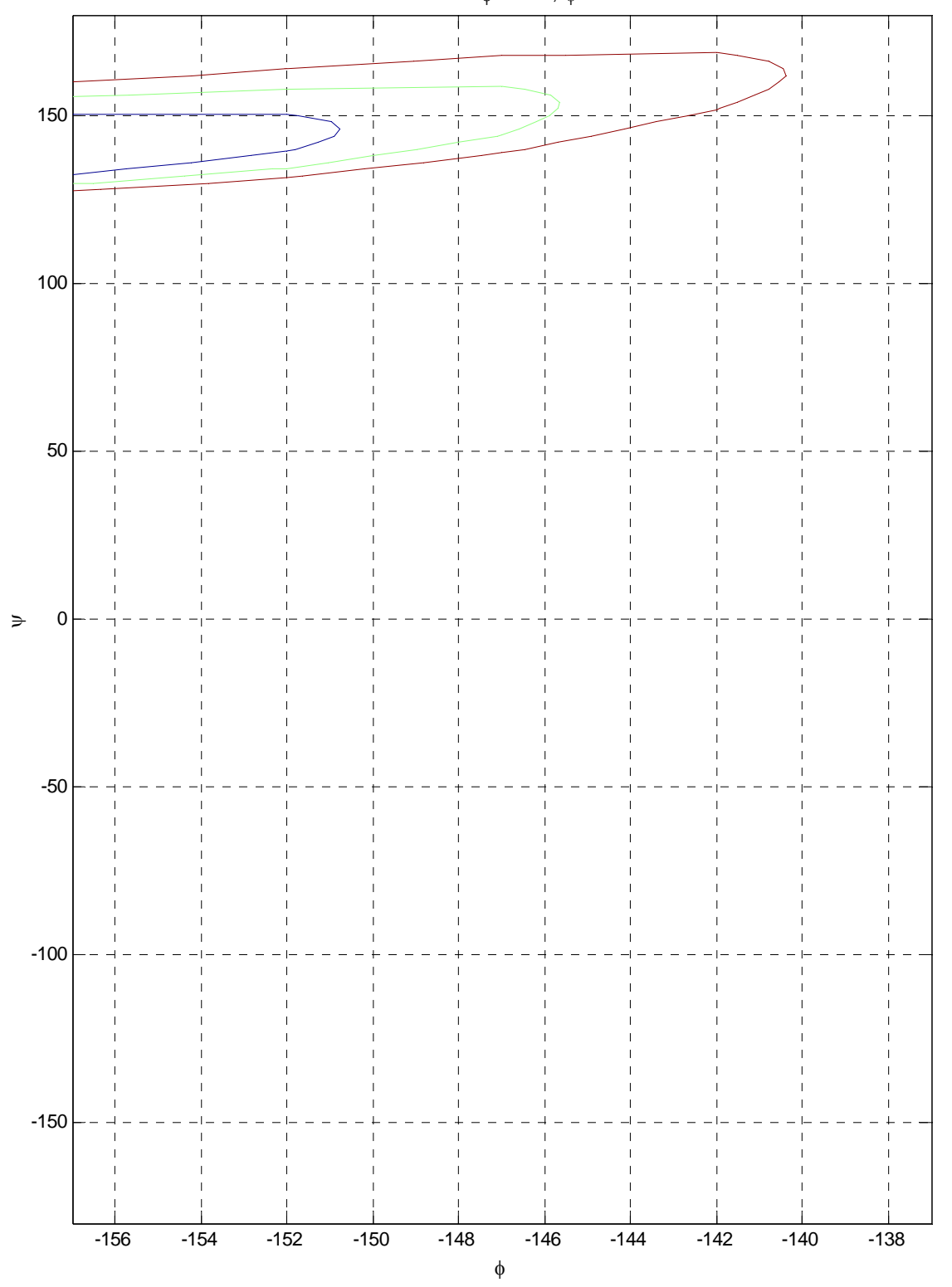

$t_{1}$ fit

Ramachandran $\chi^{2}$ map for AAA with step of 5 degrees Minimum at: $\phi=-157, \psi=140$

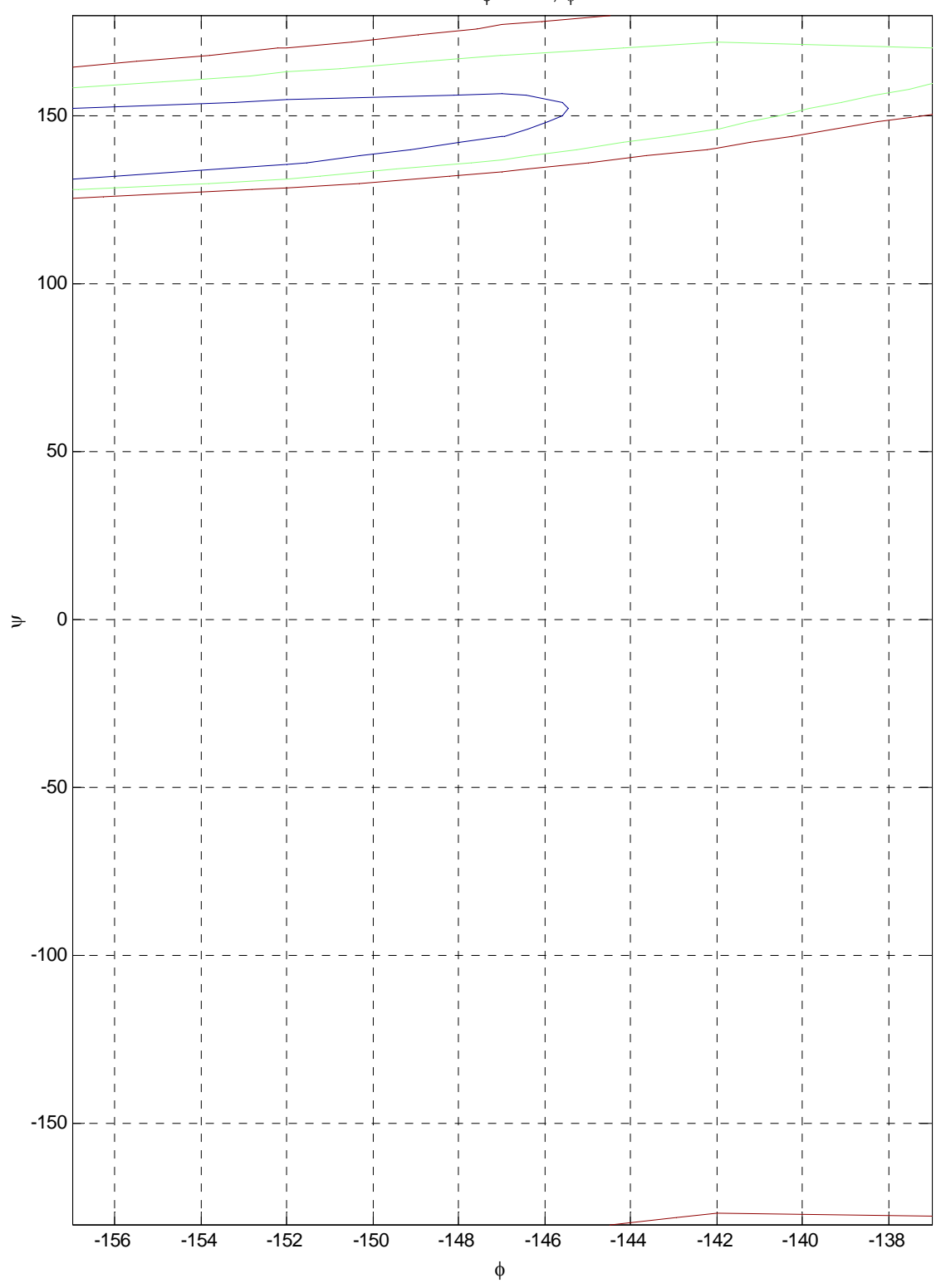




\section{$5 \times 2$ degree GEN grid}

\section{Fourier Series}

Ramachandran $\chi^{2}$ map for AAA with step of 5 degrees Minimum at: $\phi=-160, \psi=138$

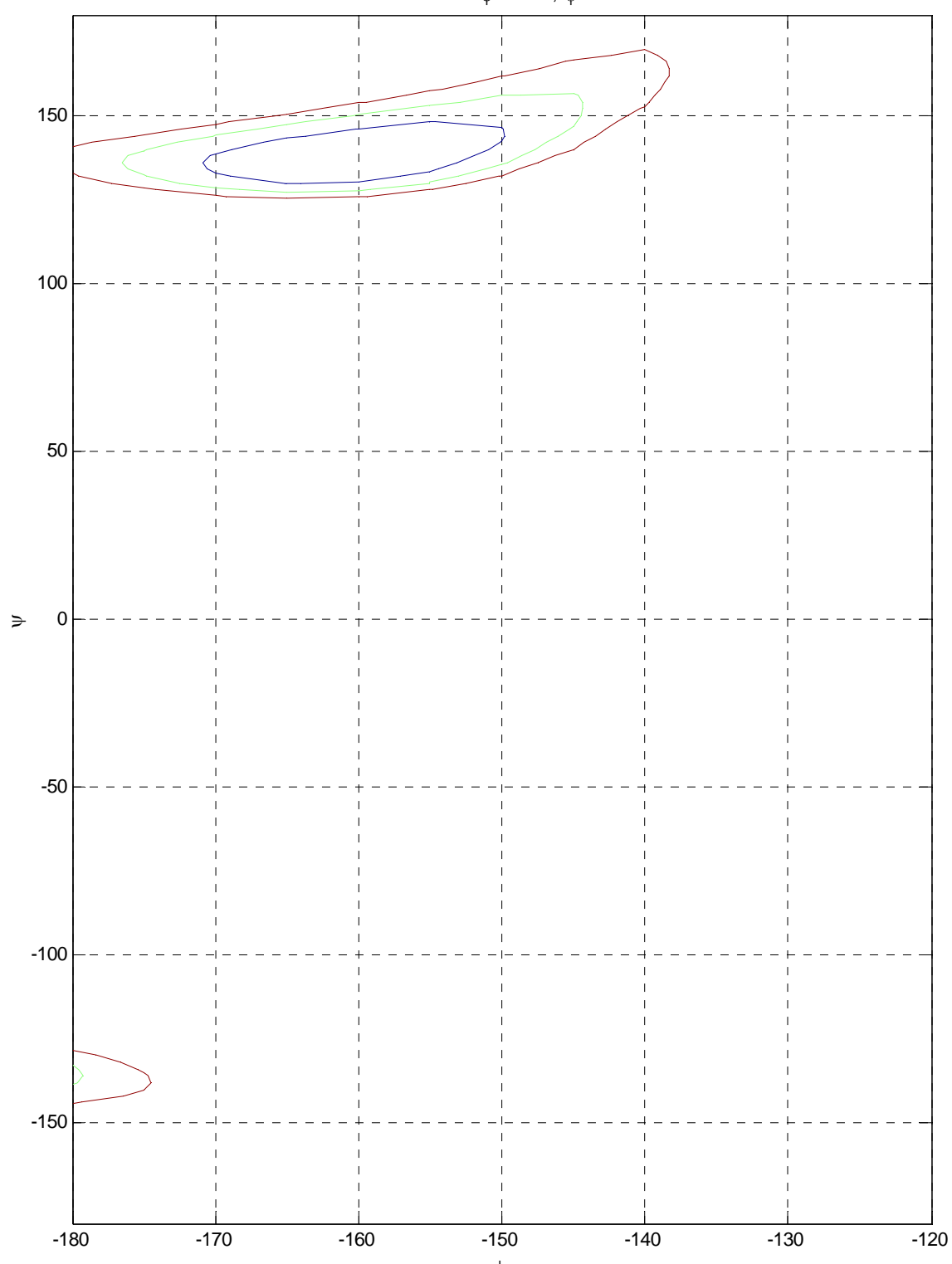

$t_{1}$ fit

Ramachandran $\chi^{2}$ map for AAA with step of 5 degrees Minimum at: $\phi=-160, \psi=138$

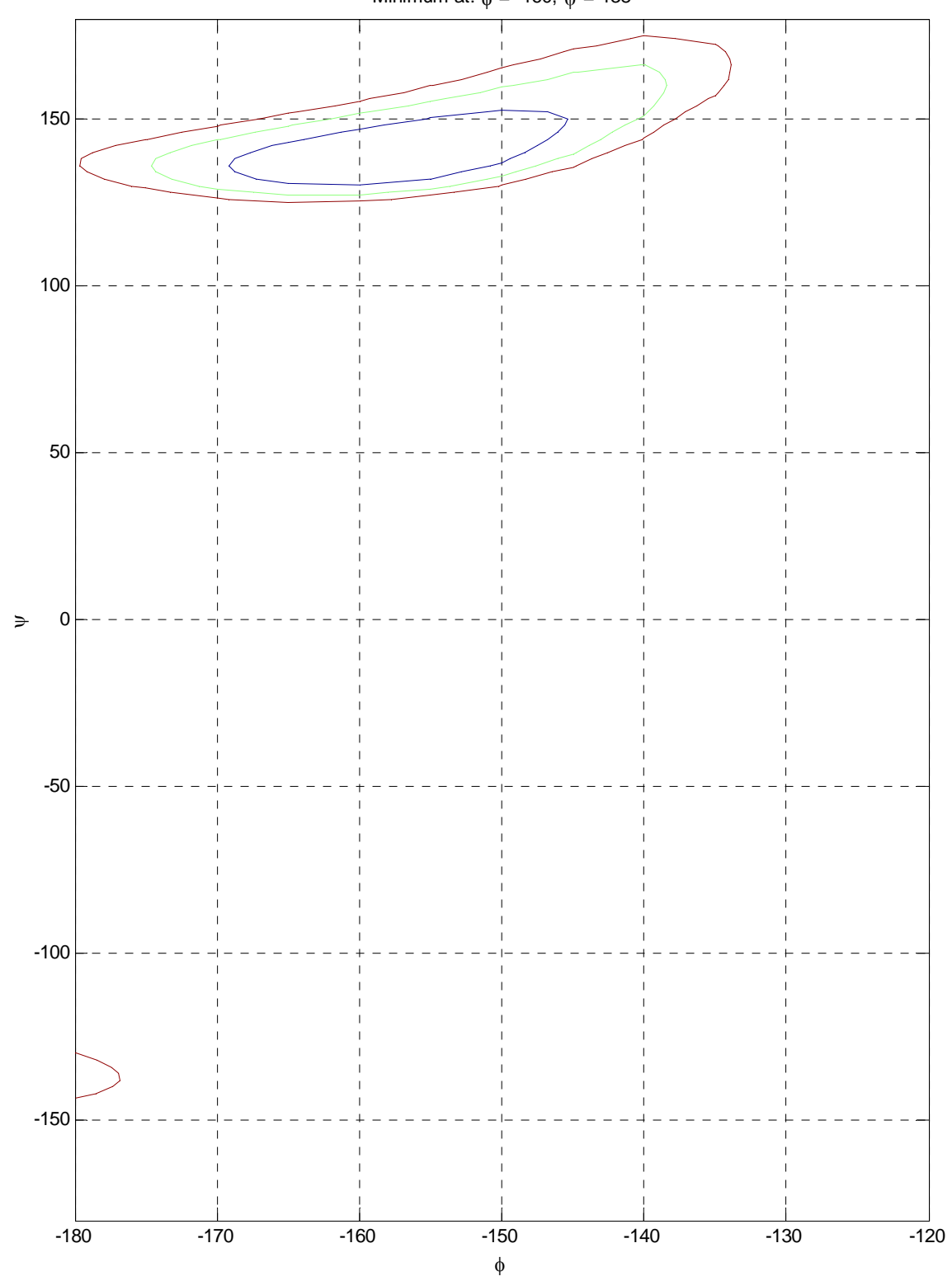


